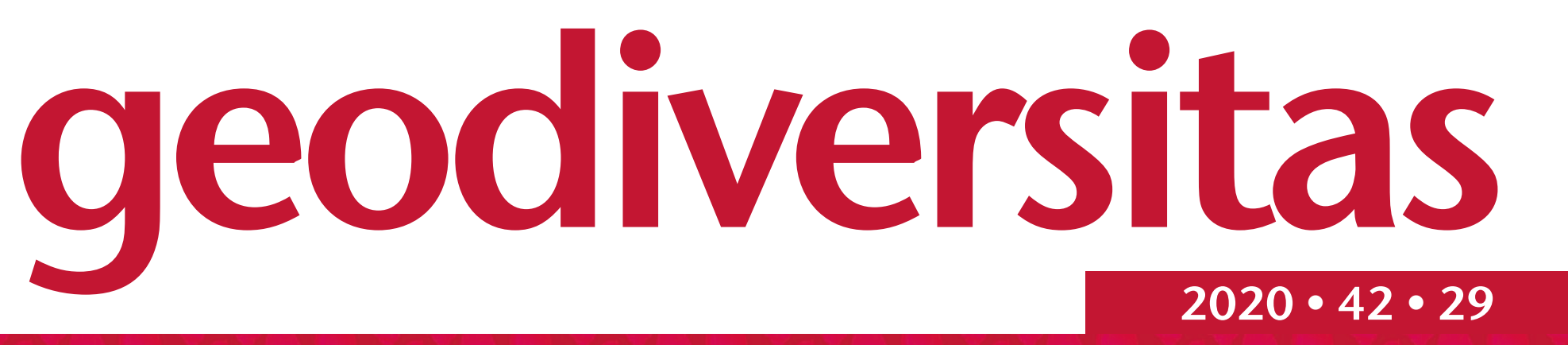

Contribution des motifs colorés résiduels dans la discrimination d'espèces nouvelles de Cryptochorda Mörch, 1858 (Mollusca: Gastropoda: Harpidae) de l'Éocène du bassin de Paris et du Cotentin

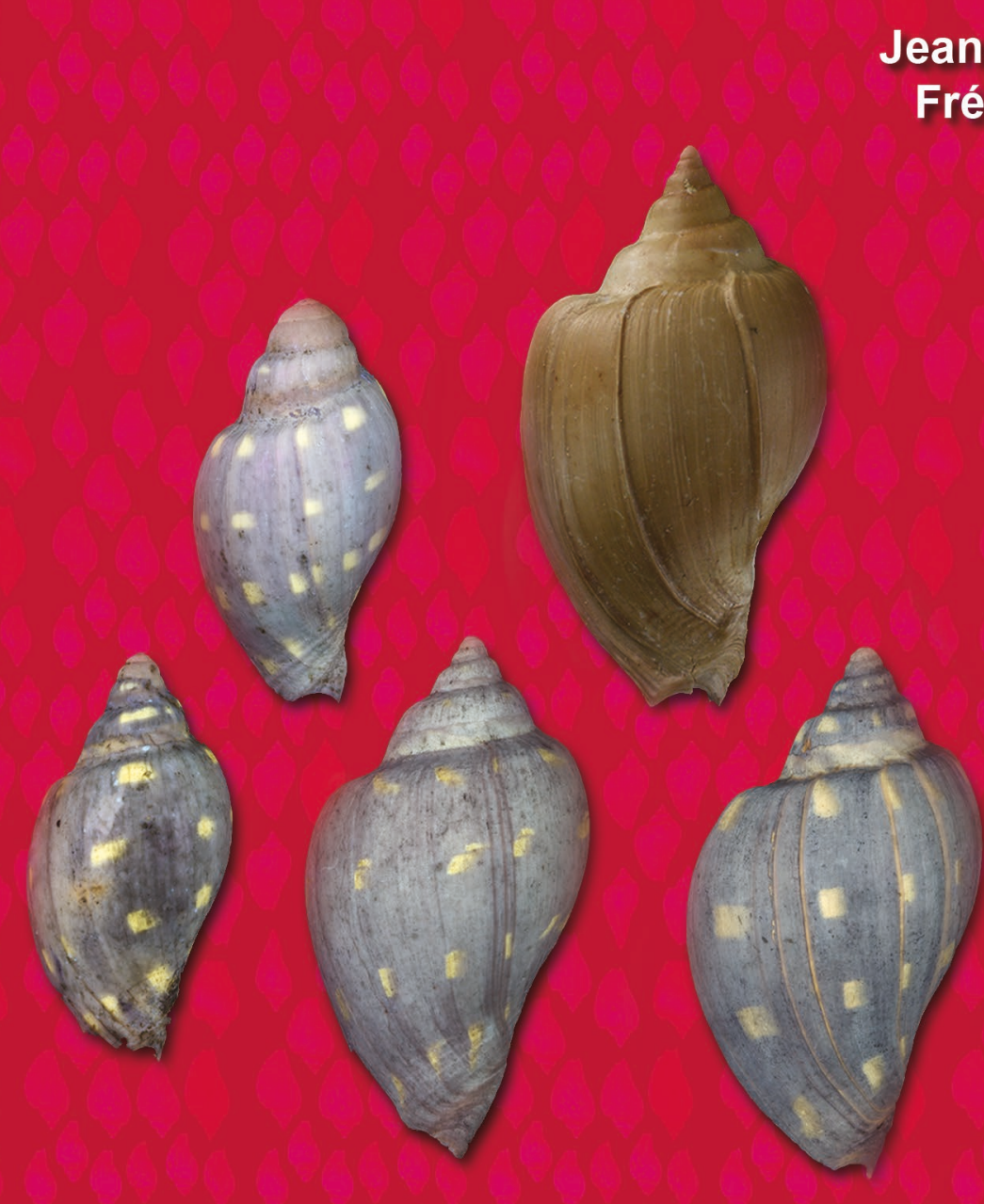


DIRECTEUR DE LA PUBLICATION: Bruno David,

Président du Muséum national d'Histoire naturelle

RÉdACTEUR EN CHEF / EDITOR-IN-CHIEF: Didier Merle

ASSISTANTS DE RÉDACTION / ASSISTANT EDITORS : Emmanuel Côtez (geodiv@mnhn.fr)

Mise en PAge / PAGE LAYOUt: Audrina Neveu, Emmanuel Côtez

COMITÉ SCIENTIFIQUE / SCIENTIFIC BOARD:

Christine Argot (Muséum national d'Histoire naturelle, Paris)

Beatrix Azanza (Museo Nacional de Ciencias Naturales, Madrid)

Raymond L. Bernor (Howard University, Washington DC)

Alain Blieck (chercheur CNRS retraité, Haubourdin)

Henning Blom (Uppsala University)

Jean Broutin (Sorbonne Université, Paris)

Gaël Clément (Muséum national d'Histoire naturelle, Paris)

Ted Daeschler (Academy of Natural Sciences, Philadelphie)

Bruno David (Muséum national d'Histoire naturelle, Paris)

Gregory D. Edgecombe (The Natural History Museum, Londres)

Ursula Göhlich (Natural History Museum Vienna)

Jin Meng (American Museum of Natural History, New York)

Brigitte Meyer-Berthaud (CIRAD, Montpellier)

Zhu Min (Chinese Academy of Sciences, Pékin)

Isabelle Rouget (Muséum national d'Histoire naturelle, Paris)

Sevket Sen (Muséum national d'Histoire naturelle, Paris)

Stanislav Štamberg (Museum of Eastern Bohemia, Hradec Králové)

Paul Taylor (The Natural History Museum, Londres)

COUVERTURE / COVER:

Made from the Figures of the article.

Geodiversitas est indexé dans / Geodiversitas is indexed in:

- Science Citation Index Expanded (SciSearch $\left.{ }^{\circledR}\right)$

- ISI Alerting Services ${ }^{\circledR}$

- Current Contents ${ }^{\circledR}$ / Physical, Chemical, and Earth Sciences ${ }^{\circledR}$

- Scopus ${ }^{\circledR}$

Geodiversitas est distribué en version électronique par / Geodiversitas is distributed electronically by:

- BioOne ${ }^{\circledR}$ (http://www.bioone.org)

Les articles ainsi que les nouveautés nomenclaturales publiés dans Geodiversitas sont référencés par / Articles and nomenclatural novelties published in Geodiversitas are referenced by:

- ZooBank ${ }^{\circledR}$ (http://zoobank.org)

Geodiversitas est une revue en flux continu publiée par les Publications scientifiques du Muséum, Paris Geodiversitas is a fast track journal published by the Museum Science Press, Paris

Les Publications scientifiques du Muséum publient aussi / The Museum Science Press also publish:

Adansonia, Zoosystema, Anthropozoologica, European Journal of Taxonomy, Naturae, Cryptogamie sous-sections Algologie, Bryologie, Mycologie.

Diffusion - Publications scientifiques Muséum national d'Histoire naturelle

CP $41-57$ rue Cuvier F-75231 Paris cedex 05 (France)

Tél.: 33 (0)1 40794805 / Fax: 33 (0)1 40793840

diff.pub@mnhn.fr / http://sciencepress.mnhn.fr

(C) Publications scientifiques du Muséum national d'Histoire naturelle, Paris, 2020

ISSN (imprimé / print): 1280-9659/ ISSN (électronique / electronic): 1638-9395 


\title{
Contribution des motifs colorés résiduels dans la discrimination d'espèces nouvelles de Cryptochorda Mörch, 1858 (Mollusca: Gastropoda: Harpidae) de l'Éocène du bassin de Paris et du Cotentin
}

\author{
Jean-Michel PACAUD \\ Centre de Recherche en Paléontologie - Paris (CR2P, UMR 7207), \\ (CNRS, MNHN, Sorbonne Université), Muséum national d'Histoire naturelle, \\ case postale 38, 57 rue Cuvier, F-75231 Paris cedex 05 (France) \\ pacaud@mnhn.fr \\ Frédéric SAUTEREAU \\ 3 clos Saint-Antoine, F-78550 Richebourg (France) \\ fredericsauterau78@gmail.com
}

Submitted on 19 September 2019 | Accepted on 4 November 2019 | Published on 17 December 2020

MOTS CLÉS

Harpidae,

Cryptochordinae, motifs colorés résiduels, caractères conchyliologiques,

Cénozoíque,

Éocene,

France,
Italie,

néotypification, espèces nouvelles.

KEY WORDS

Harpidae,

Cryptochordinae,

residual colour patterns,

shell character,

Caenozoic,

Eocene,

France,

Italy,

neotypification

new species.
Submitted on 19 September 2019 | Accepted on 4 November 2019 | Published on 17 December 2020

urn:Isid:zoobank.org:pub:ED4FE36E-3E11-4766-AEE6-35A4B45B7855

Pacaud J.-M. \& Sautereau F. 2020. - Contribution des motifs colorés résiduels dans la discrimination d'espèces nouvelles de Cryptochorda Mörch, 1858 (Mollusca: Gastropoda: Harpidae) de l'Éocène du bassin de Paris et du Cotentin Geodiversitas 42 (29): 559-595. https://doi.org/10.5252/geodiversitas2020v42a29. http://geodiversitas.com/42/29

\section{RÉSUMÉ}

L’observation sous éclairage ultraviolet de motifs colorés résiduels sur les coquilles de très nombreux gastéropodes cénozoïques fournit des caractères permettant d'affiner la description des espèces fossiles. Nous appliquons ici cette méthode à la discrimination de trois espèces de Cryptochorda de l'Éocène du bassin de Paris et du Cotentin confondues jusqu'ici avec l'espèce classique Cryptochorda (s.str.) stromboides (Hermann, 1781). Ces espèces sont nommées: Cryptochorda (s.str.) altavesna n. sp., C. (s. str.) neptis n. sp. et $C$. (s.str.) cosediensis $\mathrm{n}$. sp. La répartition stratigraphique et géographique de l'espèce type C. (s.str.) stromboides est ainsi redéfinie. Cryptochorda (s.str.) teae n. sp. est également décrite de l'Yprésien (Éocène inférieur) d'Italie. Une revue historique de la littérature portant sur la taxonomie du genre est donnée et une notice biographique est fournie pour Jean Hermann, descripteur de l'espèce type du genre Cryptochorda.

\section{ABSTRACT}

Contribution of residual colour patterns in the discrimination of new species of Cryptochorda Mörch, 1858 (Mollusca: Gastropoda: Harpidae) from the Eocene of the Paris Basin and the Cotentin peninsula. Ultraviolet light observation of residual color patterns on the shells of many Cenozoic gastropods provides characters to refine the description of fossil species. Here we apply this method to the discrimination of three species of Eocene Cryptochorda from the Paris Basin and Cotentin so far confused with the classical species Cryptochorda (s.str.) stromboides (Hermann, 1781). These species are named: Cryptochorda (s.str.) altavesna n. sp., C. (s.str.) neptis n. sp. and C. (s.str.) cosediensis n. sp. The stratigraphic and geographical distribution of type species $C$. (s.str.) stromboides is thus redefined. Cryptochorda (s.str.) teae n. sp. is also described from the Ypresian (Lower Eocene) of Italy. A histori$\mathrm{cal}$ review of the literature on the genus taxonomy is carried out and a biographical note is provided for Jean Hermann, descriptor of the type species Cryptochorda. 


\section{EXTENDED ABSTRACT}

Cryptochorda (s.str.) is a relatively uncommon genus described from the Paleocene and Eocene. Three species are known in Europe. Cryptochorda (s.str.) catharinae (Makarenko, 1976), described from the Danian (Lower Paleocene) of Luzanovka (Ukraine), and previously placed in the genus Harpa (Makarenko 1976: 143-144, pl. 13, fig. 21-24), displays a strongly ribbed shell. During the Eocene, two species are known, Cryptochorda (s.str.) stromboides (Hermann, 1781) from the Paris Basin, the type species, and Cryptochorda (s.str.) pyrenaicensis Cossmann, 1923, from the Bearn region. Cryptochorda dudariensis, was described by Strausz (1966: 134-135, pl. 18, fig. 1 and 5; text-fig. 17-18) from the middle Eocene of Dudar (Hungary), and which we believe actually belongs to the genus Pseudoliva, as shown by its well-defined spiral sulcus, typical of Pseudolividae. Cryptochorda (s.str.) stromboides (Hermann, 1781) is also reported from the Bracklesham beds (Lutetian, middle Eocene), in England (Sowerby J. de C. in Dixon 1850: 106, pl. 7, fig. 33; Morris 1854: 236; Newton 1891: 104) and the Aalter and Wemmel sands (Bruxellian, Lutetian, Middle Eocene, and Wemmelian, Bartonian, Middle Eocene, respectively) in Belgium (Nyst 1853: 382; Dewalque 1868: 201; Glibert 1933: 97, 98; 1938: 114; 1985: 336, 337). Four species occur in North America Cryptochorda (s.str.) mohri (Aldrich, 1886), Cryptochorda (s.str.) californica (Cooper, 1894), Cryptochorda (s.str.) eureia Stenzel \& Turner, 1940 and Cryptochorda (s.str.) stenostoma Stenzel \& Turner, 1940; they are described in the Claibornian (Bartonian, Middle Eocene) of Alabama (De Gregorio 1890: 106; Cossmann 1893: 38; Palmer 1937: 399), Texas, Oregon (Turner 1938: 72; Stenzel \& Turner 1940: 795; Garvie 1996: 86) and of California (Vokes 1939: 139; Weaver 1942: 499, pl. 95, fig. 19 and 23; Keen \& Bentson 1944: 129). Moreover, Garvie (1996: 86, 87, pl. 18, fig. 16) reports on the presence of Cryptochorda sp. in the Texan Claibornian (Bartonian, Middle Eocene). Traub (1981: 52, 53, pl. 10, fig. 8a, b) introduced the taxon Cryptochorda (s.str.) ventricosa in the Ilerdian (Ypresian, lower Eocene) of Austria, but it may rather be attributed to the genus Strepsidura Swainson, 1840 [type species: Murex ficulneus Holten, 1802 by monotypy, synonym of Murex turgidus Solander in Brander, 1766]. Cuvillier (1933: 54, 55, pl. 6, fig. 24 and 30) has discussed and figured a species from the Eocene of Egypt, which he labelled Cryptochorda sp. This specimen (MNHN.F.B44001) is a very badly preserved internal mould does not even support a familial attribution; this specimen is not considered in this contribution. Cryptochorda (Pseudoscapha) ogormani Cossmann, 1923 from the Cuisian (Ypresian, lower Eocene) of Gan (Pyrenees-Atlantiques), type species of the subgenus Pseudoscapha Cossmann, 1923, is characterized by its strongly bended columella and by the absence of decurrent ridges at the neck. Cryptochorda (Neocryptochorda) vientoensis Clark, 1946 from the Eocene of the area of Bolivar in Columbia (Clark \& Durham 1946: 43-44, pl. 21, fig. 17-18) type species of the subgenus Neocryptochorda Clark, 1946, is characterized by ribs on the posterior part of the whorls [and not anterior part, as mentioned by authors]. We consider that this basic feature, which we found as well in one of the new species described herein and in the species of the Paleocene of Ukraine, does not justify the creation of this subgenus. For this reason, we list this subgenus as a junior subjective synonym of Cryptochorda (s.str.). On this last point, we also report on the occurrence of Cryptochorda (Lyrianella) lyrata Nelson, 1925, type species of the subgenus Lyrianella Nelson, 1925 by original designation, characterized by a strong costulation (Nelson 1925: 432, pl. 59, figs 1-3), in the Eocene of California (Martinez Formation). The sculpture and the general shape of this subgenus are quite similar to those of the Volutidae Lyria Gray, 1847 (type species: Voluta nucleus Lamarck, 1811 by original designation [synonym of Voluta pattersonia Perry, 1811]); however it is excluded from this genus by the lack of columellar folds.

The small variations of the shells features resulted in a biased taxonomic inventory and to an under estimation of the species number in the genus Cryptochorda Mörch, 1858 from the Eocene of France. Cossmann indded wrote in 1899 (Cossmann 1899: 78), about the type species Buccinum stromboides Hermann, 1781: "[Cette espèce se trouve] aux trois niveaux du Bassin de Paris, dans le Bartonien d'Angleterre, dans le Bassin de Nantes", in 1913 (Cossmann 1913: 96) "Cette espèce, signalée aux trois niveaux éocèniques du Bassin de Paris", and in 1923 (Cossmann 1923: 25) “[C'est] une coquille très répandue dans le Bassin de Paris, aux trois niveaux de l'Éocène [...]”. More recently, Glibert (1985: 336) wrote “Cryptochorda stromboides 
a été trouvé [...] dans le bassin de Paris depuis les Sables de Cuise jusqu'aux Sables du Ruel". The results of the present study show that these observations must be reviewed. Although the similarities of the shells are flagrant, we have noticed features that can be used to distinguish the specimens. Shells are known to display only a uniformly coloured shell, from light brown, amber, to more or less intense reddish brown, and it is exceptional in the fossil record to be able to see so many specimens with residual patterns of colours under UV lighting. This discovery confirms the occurrence, in French Eocene, of five species of the Cryptochorda (s.str.) genus. The colour pattern observed by Cossmann (1923: 126) on shells of Cryptochorda (s.str.) pyrenaicensis Cossmann, 1923: "[...] porte la trace de flammules brunes, obliquement disseminées sur la région antérieure et vernissée de l'avant-dernier tour" is an artefact of fossilisation; the actual residual colour pattern of this species is very different, as will be described later on.

The species of the genus Cryptochorda (s.str.) from the Eocene of the Paris Basin are here reconsidered based on a study of over 1800 specimens, from the Museum's and private collections. Some ancient reports of Cryptochorda (s.str.) stromboides from the Cuisian (Ypresian, lower Eocene) and the Lutetian (middle Eocene) actually correspond to the new species we describe herein and which are only similar to this species by their external aspect. The Bartonian specimens from the Paris Basin and from the Loire-Atlantique region (Bois-Gouët, Saffré) are indistinguishable from Cryptochorda (s.str.) stromboides. We finally focused on the callous bulge developed on the ventral face of specimen from the Lutetian of Authevernes (Eure), observed by the second author of this work. A survey of our collections yielded numerous specimens of this type, which were sorted out searching for this distinctive character. Furthermore these specimens display residual patterns of colours under UV lighting that are lacking in Cryptochorda (s.str.) stromboides (Hermann, 1781).

Jean Hermann's collections are stored in the zoological Museum of Strasbourg (France). Until the 1870 and the annexation of Alsace-Lorraine that were considered as "Germanic" territories by Germans, the University and the Museum worked on those natural history collections. These collections were then sent to the Zoological Museum (stuffed animals) and the University of Strasbourg (botanic and geology). Only a small amount of fossils is still in the museum, but it corresponds to a collection was acquired at the end of the $19^{\text {th }}$ and beginning of the $20^{\text {th }}$. Moreover, the University's paleontological collections have no specimens from the Hermann's collection (comm. Marie-Dominique Wandhammer \& Kevin Janneau). Buccinum stromboides Hermann, 1781 is the type species of the genus Cryptochorda Mörch, 1858. We did not locate the type material of Buccinum stromboides Hermann, 1781 from the Lutetian (middle Eocene) of Courtagnon (Marne), neither at the zoological Museum nor at the University of Strasbourg, which may be irremediably lost. We provide here a description of Cryptochorda (s.str.) stromboides and designate a neotype to settle its features and clarify its taxonomic status.

\section{MATERIAL AND METHOD}

More than 1800 specimens of Cryptochorda shells have been observed and 520 specimens (260 of each species, C. (s.str.) stromboides and C. (s.str.) altavesna n. sp.) have been measured. Except for the type species Cryptochorda (s.str.) stromboides, all species studied herein display preserved residual pattern of colours. The original patterns of colours of those Cenozoic molluscs can be revealed with a sodium hypochloride bath followed by exposure to UV light. Following the pioneering works of Olsson (1967), Vokes \& Vokes (1968) and Cate (1972), numerous authors have illustrated molluscs under UV light, with more or less success. Recently (Pacaud \& Caze 2011; Pacaud \& Pons 2013; Pacaud \& Ledon 2014; Pacaud \& Cazes 2014; Pacaud \& Laporte 2015; Pacaud et al. 2015; Pacaud 2016; 2017a, b, c; 2018; Pacaud \& Vicián 2019),

KEY WORDS

Harpidae,

Cryptochordinae, residual colour patterns,

shell character,

Caenozoic

Eocene,

France,

Italy,

neotypification new species. we formalized this technique that was used for over ten years (Pacaud 2003), by redefining the experimental protocol to optimize this method. In the first step, the specimens are placed into a sodium hypochlorite concentrated aqueous solution for 24 hours. They are then placed under a long wave UV lighting (wavelength $3600 \AA$ ). The oxidation of the residual pigments provoked by the sodium hypochlorite reveals, by fluorescence under UV lighting, the pattern of colours that are invisible under natural light. The specimens are then carefully water-washed and completely dried out, to prevent the formation of sodium salts deposits on their surface. These residual patterns are light and fluorescent on a dark background. 


\section{INTRODUCTION}

Cryptochorda (s.str.) est un genre relativement rare, décrit du Paléocène et de l'Éocène. En Europe, trois espèces sont connues. Au Paléocène inférieur, Cryptochorda (s.str.) catharinae (Makarenko, 1976), décrite du Danien de Luzanovka (Ukraine) et assignée à l'origine au genre Harpa (Makarenko 1976: 143144, pl. 13, fig. 21-24), montre une coquille ornée de fortes côtes. A l'Éocène, deux espèces sont connues, Cryptochorda (s.str.) stromboides (Hermann, 1781) du bassin de Paris, l'espèce type et Cryptochorda (s.str.) pyrenaicensis Cossmann, 1923 du Béarn. Cryptochorda (s.str.) stromboides (Hermann, 1781) est également signalé des Brackleshams Beds (Lutétien, Éocène moyen) en Angleterre (Sowerby J. de C. in Dixon 1850: 106, pl. 7, fig. 33; Morris 1854: 236; Newton 1891: 104; Morley Davies 1935: 305; Tracey et al. 1996: 121, 123) [voir http://www.dmap.co.uk/fossils/bracklesham/ gast/brackgast.htm\#crystro.jpg] et dans les Sables d'Aalter (Bruxellien, Lutétien, Éocène moyen) et de Wemmel (Wemmelien, Bartonien, Éocène moyen) en Belgique (Nyst 1853: 382; Dewalque 1868: 201; Glibert 1933: 97-98; 1938: 114; 1985: 336-337; Geys \& Marquet 1983: 118). Quatre espèces sont également connues d'Amérique du Nord, Cryptochorda (s.str.) mohri (Aldrich, 1886), C. (s.str.) californica (Cooper, 1894), C. (s.str.) eureia Stenzel \& Turner, 1940 et C. (s.str.) stenostoma Stenzel \& Turner, 1940, sont décrites du Claibornien (Bartonien, Éocène moyen) d'Alabama (De Gregorio 1890: 106; Cossmann 1893: 38; Palmer 1937: 399), du Texas et de l'Oregon (Turner 1938: 72; Stenzel \& Turner 1940: 795; Garvie 1996: 86) et enfin de Californie (Vokes 1939: 139; Weaver 1942: 499, pl. 95, fig. 19 et 23; Keen \& Bentson 1944: 129). Par ailleurs, Garvie (1996: 86-87, pl. 18, fig. 16) signale également Cryptochorda sp. du Claibornien (Bartonien, Éocène moyen) du Texas. Cryptochorda dudariensis, décrit par Strausz (1966: 134-135, pl. 18, fig. 1 et 5; text-fig. 17-18) de l'Éocène moyen de Dudar (Hongrie) est en fait une Pseudoliva, comme le montre son sulcus spiral, caractéristique des Pseudolividae [voir https://www.fossilshells. nl/taxon_columbellidae.html]. Traub (1981: 52-53, pl. 10, fig. 8a, b) a introduit le taxon Cryptochorda (s.str.) ventricosa de l'Ilerdien (Yprésien, Éocène inférieur) d'Autriche, mais il s'agit plus vraisemblablement d'une espèce appartenant au genre Strepsidura Swainson, 1840 (espèce type: Murex ficulneus Holten, 1802 par monotypie, synonyme de Murex turgidus Solander in Brander, 1766). Cuvillier (1933: 54-55, pl. 6, fig. 24 et 30) a discuté et figuré une espèce de l'Éocène d'Égypte sous le nom Cryptochorda sp., mais le spécimen que nous avons examiné (MNHN.F.B44001), est représenté par un moule interne si mal conservé que ce volume calcaire ne permet même pas une assignation familiale; nous n'en tenons évidemment pas compte. Cryptochorda (Pseudoscapha) ogormani Cossmann, 1923 du Cuisien (Yprésien, Éocène inférieur) de Gan (Pyrénées-Atlantiques), espèce type du sous-genre Pseudoscapha Cossmann, 1923, est caractérisée (Cossmann 1923: 126-127, pl. 8, fig. 11-13) par sa columelle fortement coudée et par l'absence, au niveau du cou, de stries décurrentes (MNHN.F.J10900, coll. O'Gorman). Cryptochorda (Neocryp- tochorda) vientoensis Clark, 1946 de l'Éocène des environs de Bolivar en Colombie (Clark 1946: 43, 44, pl. 21, fig. 17-18), espèce type du sous-genre Neocryptochorda Clark, 1946, est caractérisée par la présence de côtes sur la partie postérieure des tours [et non antérieure comme l'écrivent les auteurs de ce sous-genre]. Selon nous, cette simple particularité, que nous retrouvons chez l'une des espèces nouvelles que nous décrivons ici et sur l'espèce du Paléocène d'Ukraine, ne justifie pas l'utilité de ce sous-genre que nous considérons comme un synonyme subjectif plus récent de Cryptochorda (s.str.). Sur ce dernier point, nous relevons également la présence à l'Éocène de Californie (Martinez Formation) de l'espèce Cryptochorda (Lyrianella) lyrata Nelson, 1925, espèce type du sous-genre Lyrianella Nelson, 1925 par désignation originale, caractérisée par une forte costulation (Nelson 1925: 432, pl. 59, fig. 1-3). La sculpture et la forme générale de ce sous-genre sont assez similaires à celles observées chez le Volutidae Lyria Gray, 1847 (espèce type: Voluta nucleus Lamarck, 1811 par désignation originale [synonyme de Voluta pattersonia Perry, 1811]); l'absence de plis columellaires l'exclue cependant de ce genre.

La faible variation des caractères de ces coquilles a donné lieu à un inventaire taxonomique biaisé et à une sous-estimation spécifique du genre Cryptochorda Mörch, 1858 à l'Éocène en France. En effet, à propos de l'espèce type Buccinum stromboides Hermann, 1781, Cossmann écrit en 1899 (1899: 78): "[Cette espèce se trouve] aux trois niveaux du Bassin de Paris, dans le Bartonien d'Angleterre, dans le Bassin de Nantes", et en 1913 (1913: 196): "Cette espèce, signalée aux trois niveaux éocèniques du Bassin de Paris [...]», puis encore en 1923 (1923: 125): «[C’est] une coquille très répandue dans le Bassin de Paris, aux trois niveaux de l'Éocène [...]». Plus récemment Glibert (1985: 336) écrit également: «Cryptochorda stromboides a été trouvé [...] dans le bassin de Paris depuis les Sables de Cuise jusqu'aux Sables du Ruel». Au regard de l'étude que nous venons d'effectuer, ces commentaires méritent d'être largement revus. Bien que ces exemplaires présentent une évidente similitude conchyliologique, nous avons identifié des caractères qui peuvent être utilisés pour séparer ces spécimens. Par ailleurs, sur des coquilles réputées pour n'avoir seulement qu'une teinte uniforme allant d'un brun clair, ambré, à une teinte brun-rougeâtre plus ou moins intense et, fait exceptionnel dans le registre fossile, très souvent préservée, des motifs colorés résiduels observés sous éclairage UV ont été mis en évidence. Cette découverte nous permet maintenant de signaler la présence à l'Éocène en France de cinq espèces appartenant au genre Cryptochorda (s.str.). Le motif de couleur qu'a cru discerner Cossmann (1923: 126) sur des coquilles de C. (s.str.) pyrenaicensis Cossmann, 1923: «[...] porte la trace de flammules brunes, obliquement disséminées sur la région antérieure et vernissée de l'avant-dernier tour " n'est qu'un artefact de fossilisation; le véritable motif résiduel de couleur de cette espèce est en fait très différent comme nous le verrons ci-après.

Le contenu spécifique du genre Cryptochorda (s.str.) à l'Éocène du bassin de Paris est ici redéfini après l'étude de plus de 1800 exemplaires des collections du Muséum et de collections privées. Certaines citations au Cuisien (Yprésien, 
C. (s.str.) altavesna n. sp.

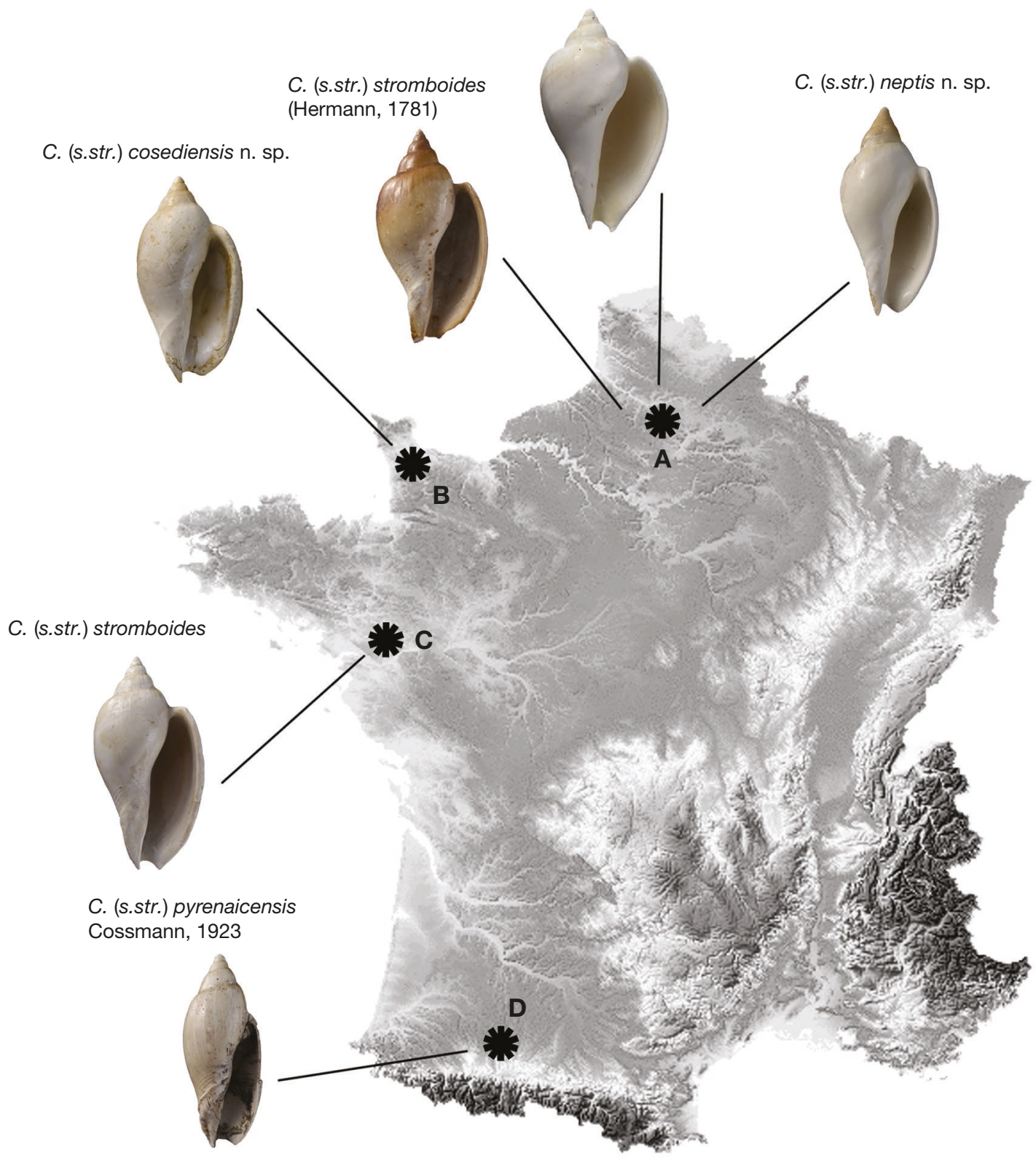

FIG. 1. - Répartition géographique des taxa étudiés : A, bassin de Paris; B, Cotentin; C, Loire-Atlantique; D, bassin d’Aquitaine (voir Cadre géographique).

Éocène inférieur) et au Lutétien (Éocène moyen), par les anciens auteurs, sous le nom spécifique de Cryptochorda (s.str.) stromboides, concernent en fait des espèces nouvelles que nous décrivons ici et qui ne partagent avec le précédent taxon que son aspect extérieur. Les spécimens du Bartonien du bassin de Paris et de Loire-Atlantique (Bois-Gouët à Saffré, PierreAiguë à Saint-Aignan-Grandlieu et le couloir «Le Maupas - les Étangs" à la Limouzinière) sont quant à eux indiscernables de C. (s.str.) stromboides. Notre attention aura finalement été attirée par l'observation du second auteur d'un important bourrelet calleux sur la face ventrale de spécimens du Lutétien de Authevernes (Eure). Nous avons alors pu retrouver de très nombreux autres spécimens dans nos collections, puis trier de nouveaux matériaux avec un oil averti. Par ailleurs, ces spécimens présentent, sous éclairage UV, des motifs colorés résiduels particuliers, totalement absents chez l'espèce Cryptochorda (s.str.) stromboides (Hermann, 1781).

Les collections Jean Hermann sont conservées au Musée zoologique de la ville de Strasbourg, qui, voulant profiter de la loi du 11 floréal an X votée le 1er mai 1802 sous le consulat de Napoléon, portant qu'il serait établi trois écoles d'histoire naturelle, sollicita et obtint l'autorisation d'acheter le cabinet Hermann pour la somme de 40000 francs. Celui-ci, considérablement enrichi par les soins de son gendre, le professeur Hammer, de Colmar, qui en fut nommé le conservateur. Cette acquisition était, pour les héritiers, une faible indemnité des quarante années passées et de l'argent dépensé par leur père à augmenter ses collections. Les collections d'histoire naturelle étaient utilisées par le Musée et l'université jusqu'en 1870. Lors de l'annexion en 1870 de l'Alsace-Lorraine, territoires 
considérés par les Allemands comme "germaniques", ces collections ont été réparties entre le Musée zoologique [spécimens naturalisés] et l'université de Strasbourg [botanique et géologie au sens large]. Il reste une petite quantité de fossiles au Musée, mais il s'agit d'une collection acquise fin XIX ${ }^{\mathrm{e}}$ début XXe. Par ailleurs, les collections de paléontologie de l'Université ne contiennent aucun spécimen provenant de la collection Hermann (comm.Marie-Dominique Wandhammer \& Kévin Janneau). Buccinum stromboides Hermann, 1781 est l'espèce type du genre Cryptochorda Mörch, 1858. Nous n'avons malheureusement pas retrouvé, ni au Musée zoologique ni à l'université de Strasbourg, le matériel type du taxon Buccinum stromboides Hermann, 1781 provenant du Lutétien (Éocène moyen) de Courtagnon (Marne) qui semble irrémédiablement perdu. Nous redonnons une description de Cryptochorda (s.str.) stromboides et nous désignons un néotype pour en fixer les caractères et clarifier son statut taxonomique.

\section{MATÉRIEL ET MÉTHODE}

Nous avons examiné plus de 1800 exemplaires de coquilles de Cryptochorda et avons procédé à des mesures sur 520 spécimens (260 de chacune des espèces $C$. (s.str.) stromboides et C. (s.str.) altavesna n. sp.). Hormis l'espèce type Cryptochorda (s.str.) stromboides, les espèces étudiées ici montrent toutes des motifs de couleur résiduel préservés. Les motifs colorés disparus de ces mollusques cénozoïques peuvent être révélés par un bain d'hypochlorite de sodium suivi d'une exposition sous éclairage ultraviolet. A la suite des travaux initiés par Olsson (1967), Vokes \& Vokes (1968) et Cate (1972), de nombreux auteurs ont publié des illustrations de mollusques pris sous éclairage ultraviolet, avec plus ou moins de réussite. Nous avons redéfini le protocole expérimental permettant d'optimiser cette méthode depuis près de dix-sept ans (Pacaud 2003), et nous avons récemment (Caze et al. 2010, 2011a, b; Pacaud \& Caze 2011; Pacaud \& Pons 2013; Pacaud \& Ledon 2014; Pacaud \& Cazes 2014; Pacaud \& Laporte 2015; Pacaud et al. 2015; Pacaud 2016; 2017a, b, c; 2018; Pacaud \& Vicián 2019) formalisé cette approche. Dans un premier temps, les échantillons sont placés dans une solution aqueuse concentrée d'hypochlorite de sodium pendant une durée de 24 heures. Ils sont ensuite, dans un second temps, disposés sous une lampe émettant une lumière UV à ondes longues (longueur d'onde de 3600 Å). L'hypochlorite de sodium entraîne une oxydation des résidus de pigments qui permet, sous éclairage UV, de rendre fluorescents les motifs colorés invisibles en lumière naturelle. Les échantillons doivent être soigneusement lavés à l'eau avant d'être séchés pour éviter la formation de dépôts de sels de sodium à la surface de la coquille. Ces motifs résiduels apparaissent clairs et fluorescents sur un fond sombre.

\section{CADre GÉOGRAPHIQUe (Fig. 1).}

Le matériel étudié, des spécimens des collections du MNHN ainsi que des exemplaires appartenant à plusieurs collections privées, représente plus de 1800 spécimens provenant de 62 gisements fossilifères de l'Éocène du bassin de Paris, du
Cotentin et de la Loire-Atlantique. Les sites mentionnés se répartissent ainsi :

- dans le département de l'Oise (Cuise-la-Motte [La Buttedes-Usages], Cinqueux, Parnes, Chaumont-en-Vexin [Carrière de la Laiterie, Carrière Darcy], Mouchy-le-Châtel, Cauvigny [Fercourt, Châteaurouge], Ully-Saint-Georges [Laluet], Ponchon, Monts, Saint-Félix, Boury-en-Vexin, Saint-Waast-lèsMello [Barisseuse], Baron [Sablière Heudebert]);

- dans le département du Val d'Oise, au sud-ouest de Beauvais (Chaussy [Les Garennes], Saint-Witz [Le Guépelle], Haravilliers [Le Quoniam]);

- dans le département des Yvelines (Thiverval-Grignon, Villiers-Saint-Frédéric [La Butte-Saint-Léonard], Beynes [La Remise à Boucher], Saulx-Marchais, Montainville, Maulettte);

- dans le département de l'Eure (Authevernes, Cahaignes [Requiécourt], Vexin-sur-Epte [Guitry], Fontenay-en-Vexin [Le Bois-du-But], Gisors [Mont-de-Magny]);

- dans le département d'Eure-et-Loir (Berchères-sur-Vesgre [La Ville-l'Evêque]);

- dans le département de l'Aisne (Saint-Gobain, Mons-enLaonnois, Laon, Monampteuil, Laversine, Mercin-et-Vaux, Lizy, Bruyères-et-Montbérault, Crouy, Bézu-le-Guéry);

- dans le département de la Seine-et-Marne (Dhuizy, Vendrest, La Ferté-sous-Jouarre, Mary-sur-Marne, Le Limon, Sainte-Aulde [Caumont]);

- dans le département de la Marne (Courtagnon, Damery, Fleury-la-Rivière, Chamery, Ventheuil [Arty], Montmirail, Boursault, Mécringes, Nantheuil-la-Fôret);

- dans le département de la Manche (Fresville, HauttevilleBocage);

- dans le département de la Loire-Atlantique (Saffré [BoisGouët], Saint-Aignan-Grandlieu [Pierre-Aiguë], le couloir «Le Maupas - les Étangs» [La Limouzinière]);

- et dans le département des Pyrénées-Atlantiques (Gan [la Tuilerie]).

Les spécimens décrits ici sont conservés dans les collections de Paléontologie du Muséum national d'Histoire naturelle (Paris), de l'Istituto e Museo di Geologia dell'Universita de Padova (Italie) et du Museo di Archeologia e Scienze Naturali «G. Zannato» de Montecchio Maggiore (Italie).

\section{ABRÉVIATIONS}

MNHN.F Muséum national d'Histoire naturelle, collection de paléontologie (Paris, France).

MGP-PD Istituto e Museo di Geologia dell'Universita (Padova, Italie).

MCZ Museo di Archeologia e Scienze Naturali «G. Zannato » (Montecchio Maggiore, Italie).

UBT Université Bordeaux-1 (Talence, France).

RETOUR AUX RACINES...

De Férussac (1822: 555) écrit dans le Dictionnaire classique d'Histoire naturelle: "[...] le Buccinum stromboides, que l'on trouve en Champagne avec ses couleurs, comme s'il sortait de la mer [...]». La richesse en fossiles du Lutétien du bassin de Paris et sa qualité de préservation attire très tôt l'intérêt 
des naturalistes pour cette coquille élégante, à test porcelané, extrêmement bien préservée et conservant très souvent, comme l'écrit de Férussac, sa couleur d'origine; une teinte uniforme allant d'un brun clair à une teinte brun-rougeâtre plus ou moins intense.

Nous devons la description de l'espèce Buccinum stromboides à Jean Hermann, professeur à Strasbourg, mais de nombreux auteurs se trompent sur l'auteur de cette espèce et l'attribue soit à Gmelin (de Roissy 1805: 29; Dillwyn 1817: 581; Lamarck 1822: 279; von Schmidt 1846: 163; Fritel 1886: pl. 3, fig. 31; Palmer 1977: 14), soit à Lamarck (Parkinson 1811: 58 ; 1833: 58; Isensee 1833: 40 ; Swainson 1840: 123 avec, par ailleurs, l'orthographe subséquente incorrecte Buccinum strombioïdes; d'Orbigny 1850: 320; Watelet 1855: 58; Pictet 1857: 44; Winkler 1868: 49; Fischer 1883: 605), soit à Chemnitz (Cossmann 1913: 196), voire à Hermannsen (Palmer 1937: 399; Weaver 1942: 499; Clark 1946: 43; Squires 1984: 35) [pour ce dernier il s'agit probablement d'une méprise due à l'abréviation «Herm. » utilisée par certains auteurs pour Hermann et non pour Hermannsen]. Des réseaux internationaux tels que le GBIF: Global Biodiversity Information Facility (système mondial d'information sur la biodiversité) [https:/www.gbif.org/en/species/9315179], le WMSDB: Worldwide Mollusc Species Data Base [http:// www.bagniliggia.it/WMSD/HtmSpecies/5350000810.htm], ou encore the Fossilworks PaleoDB portal [http://fossilworks. org/bridge.pl?a=taxonInfo\&taxon_no=158390] s'en font malheureusement encore l'écho...

Jean Hermann (Fig. 2) est né à Barr en Alsace, le 31 décembre 1738 , où son père est pasteur luthérien. Il entre à 15 ans à l'université de Strasbourg et y étudie brillamment les lettres et les sciences. Mais sa préférence se tourne vers la botanique et l'histoire naturelle enseignée en médecine. Il soutient deux thèses, en 1762 sur la cardamine et en 1763 sur la rose. Ensuite, il visite à Paris les cabinets d'histoire naturelle (Lauth 1801), notamment ceux du Jardin du roi, de Jean de Jullienne (16861766), de Henry-Louis Duhamel du Monceau (1700-1782), de Marie-Félicité de Boisjourdain, née Marie-Félicité de Belloy (? -1765), de la bibliothèque de Sainte-Geneviève et du séminaire de Saint-Sulpice. Hermann rend également visite à Dezallier d'Argenville en 1763 (Fig. 3A), un des grands intellectuels et collectionneurs du XVIII siècle, qui «habitait rue du Temple, la première porte cochère après la rue Pastourelle» et dans ses notes il écrit: «M. d'Argenville est un homme âgé, roux, il a l'ouïe grave, est fort affable et montre volontiers ses curiosités, mais il en fait grand cas et dit à chaque pièce combien il lui a coûté d'argent et de peine pour l'avoir » (Wickersheimer 1921). Il acquiert des spécimens pour son propre cabinet d'histoire naturelle qu'il ouvre dès 1764 , à son domicile place SaintThomas, en y donnant un enseignement d'histoire naturelle. Grâce à Jacques Reinbold Spielmann (1722-1783), professeur de botanique, de chimie et matière médicale ainsi que directeur du Jardin botanique de la ville de Strasbourg, Jean Hermann est nommé professeur extraordinaire de médecine à l'université de Strasbourg le 19 novembre 1769. Il obtient la chaire de philosophie le 12 septembre 1778 et en 1782 , la chaire de pathologie. Après le décès de Spielmann, il lui

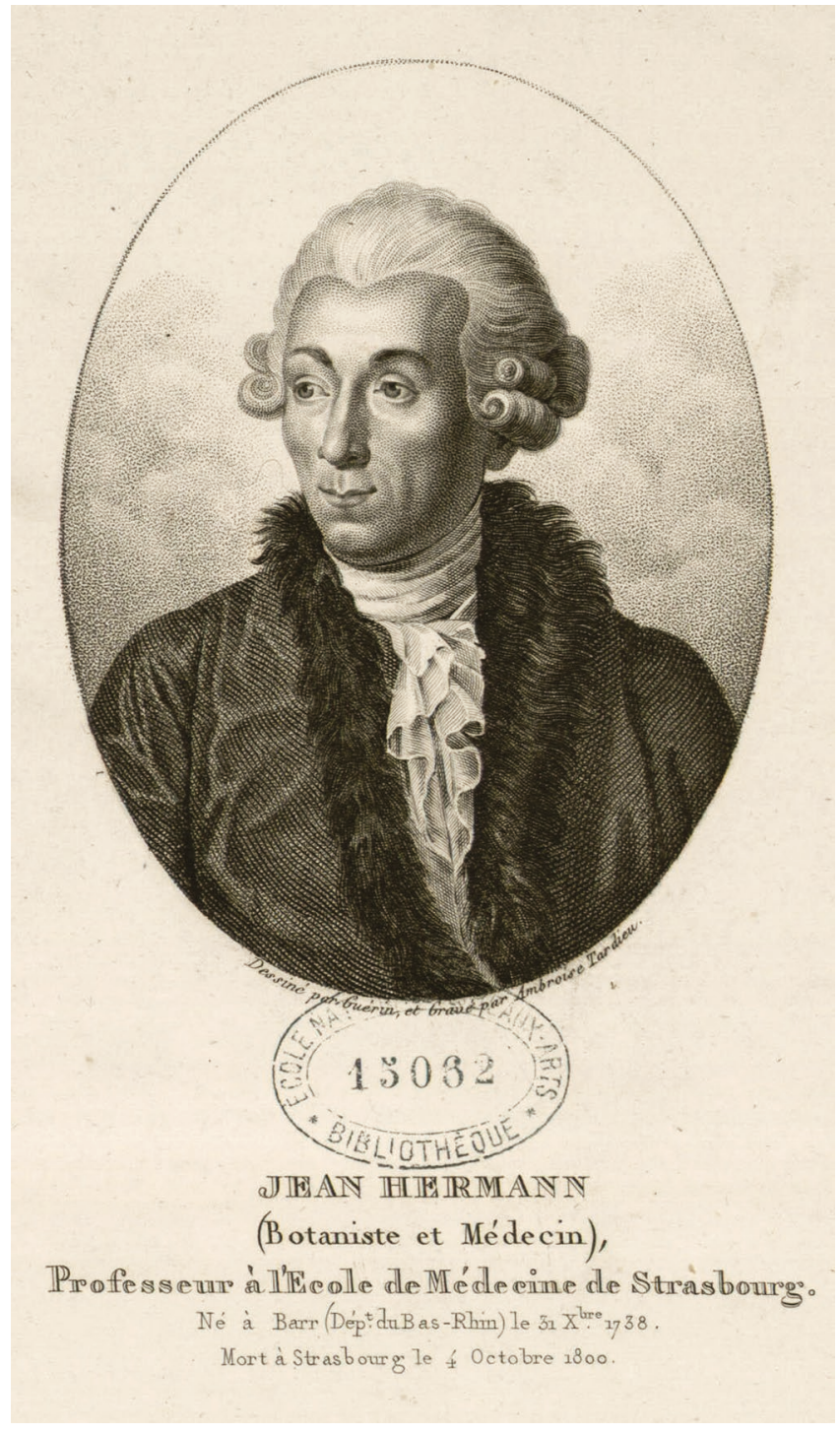

FIG. 2. - Portrait de Jean Hermann (1738-1800), dessiné par Charles Guerrin et gravé par Ambroise Tardieu (frontispice des Observationes zoologicae publié par Hermann en 1804).

succède le 24 janvier 1784 . C'est un botaniste réputé à tel point que ses recherches le font surnommer «le Linné du Rhin ». Il développe l'enseignement de la zoologie et utilise les spécimens de ses propres collections pour illustrer ses cours à l'université et puise la matière de son enseignement dans sa bibliothèque privée (Lescure et al. 2009). Ses collections et sa bibliothèque, riche de 20000 volumes, sont à l'origine du Muséum d'histoire naturelle de Strasbourg, où son cabinet d'histoire naturelle a été recréé. Barthélémy Faujas de SaintFond, professeur et administrateur au Muséum national d'Histoire naturelle de Paris et premier titulaire de la chaire de géologie, nota ce qu'il vit en traversant l'Est de la France dans une lettre (Paris, Archives nationales, série AJ 15-581) écrite de Strasbourg le 14 floréal (avril 1798) au cours d'un «voyage d'observation » qu'il avait entrepris, à la demande du directoire, dans l'est de la France et en Allemagne. Elle fut lue à ses collègues administrateurs du Muséum dans la séance du 24 floréal (Boyer 1976), il y écrivait notamment: «[...] Me 
voici à Strasbourg. J'ai déjà passé trois heures dans le riche cabinet d'Herman [sic], où il y a de tout, et des choses rares, une tortue que je crois neuve et qui sera dessinée pour le Citoyen Lacépède, ainsi qu'un poisson et deux serpens [sic]. Beaucoup de coquilles pétrifiées rares, des minéraux précieux, des pierres de toute espèce composent ce savant cabinet, où tous les échantillons sont en général très bien étiquetés [...]». Le médecin Thomas Lauth (1801) publia une notice sur la vie de son ami Hermann, et écrira qu'il mourut le 4 octobre 1800 des suites d'un refroidissement, après une excursion botanique dans les environs d'Haguenau.

Jean Hermann $(1781: 53,54)$ ne donnera la description que d'une seule espèce de mollusque fossile. Il décrit et figure (Fig. 3C) l'espèce Buccinum stromboides, dans un petit essai sur la Conchyliologie publié dans la revue allemande Der Naturforscher, dont l'éditeur est un ami, en ces termes (traduit de l'allemand) : «Un Buccinum, qui a encore gardé l'éclat considérable et la couleur, se trouvait parmi les coquilles d'escargot calcifiés de Courtagnon. Elle ressemble au genre Strombus. En vue extérieure elle se rapproche de Strombus gibberulus. Mais la coupure de la lèvre manque dans sa totalité et elle porte le caractère absolu du genre Buccinum. Il y a cinq à six tours de spire qui sont légèrement bombés mais lisses. Le ventre et deux fois plus long que la partie spirale, lisse aussi, à l'exception de quelques côtes, qui se prolongent en longueur derrière la lèvre, en formant la délimitation de la lèvre. Il y a également six lignes étroites qui tournent obliquement à la base de l'escargot. Là où la lèvre se rapproche du deuxième tour, elle est légèrement séparée par une concavité. La couleur est d'un marronjaune, là où la peau superficielle s'est décollée, la couleur est plus claire, d'un blanc cassé. Mr. Le Professeur Wolph Murran l'a reconnu comme une espèce non encore décrite par Ritter. On peut la classer avec Buccinum glabratum, en ajoutant la description suivante: testa glabra, anfractibus distinctis, ventre retro labrum superne solutum porcato, ad basin oblique striato".

En premières pages blanches de son exemplaire de L'histoire naturelle éclaircie dans deux de ses parties principales. La lithologie et la conchyliologie [...] de Dezallier d'Argenville, publié en 1742, Jean Hermann écrit: «La troisième édition par Mr. Favanne de Moncervelle, Père \& fils, Paris, 1780. Chez de Bure. Avec 83 planches mais petites $\&$ n'égalant pas les présentes pour la beauté, de beaucoup près". (Fig. 3B). Il ne remarquera pas cependant, lors de la description en 1781 de son Buccinum stromboides, que cette espèce était figurée dans cet ouvrage de 1780 (pl. 66, fig. I10). Favanne de Moncervelle (1784: 490, $\mathrm{n}^{\circ}$ 2158) l'identifie comme «Testacites fossiles de Courtagnon, dont [...] Buccinites appellé le Buccin d'Émail».

\section{LES PREMIĖRES OBSERVATIONS}

\section{ET FIGURATIONS DE L'ESPÈCE}

Cryptochorda (s.str.) stromboides (Hermann, 1781), a été observée très tôt; nous en donnons ici quelques exemples marquants, dont certains publiés bien avant sa description en 1781 :
- Dès le XVIe siècle, dans les écrits de Bernard Palissy (1510-1590), les fossiles cénozoïques du bassin de Paris sont cités dans ses Discours admirables de la nature publiés en 1580 à Paris. Esprit curieux et innovant, figure notable de la Renaissance française, Palissy est surtout connu pour ses travaux de céramiste et produit ses premières pièces de vaisselle décorative, non culinaire, à décor animalier, les « rustiques figulines». Cependant, ce sont sur les céramiques post-palisséennes, attribuées à de proches successeurs ou à des « suiveurs » du XVIII siècle (Plaziat 2011, 2019), que l'on peut observer de nombreux coquillages actuels et fossiles qui constituent des reliefs estampés dans la surface du plat, à partir de moulages extrêmement fidèles. Brongniart (1844) a été le premier à identifier les fossiles de ces décors comme des coquilles de l'Éocène du Bassin parisien. Certains de ces plats comportent des moulages de coquilles fossiles du bassin de Paris parmi lesquels il nous semble reconnaitre quatre exemplaires de Cryptochorda (s.str.) stromboides (Fig. 4A).

- En 1765, dans son Locupletissimi rerum naturalium thesauri accurata descriptio, Albertus Seba (1665-1736), zoologiste et pharmacien hollandais, célèbre pour son cabinet d'histoire naturelles et de curiosités, donne une figure (Seba 1765: 126, pl. 106, fig. 22) d'un exemplaire (Fig. 4B) qu'il classe dans les «Buccinites".

- En 1767 (Romé de l'Isle 1767: 97, 98), Romé de l'Isle publie un Catalogue systématique et raisonné des curiosités de la nature et de l'art, un catalogue de vente du cabinet d'histoire naturelle de Pedro Francisco Dávila (1713-1785), un naturaliste équatorien, illustrateur spécialisé dans les invertébrés marins, les minéraux et les fossiles et fondateur du Real Gabinete de Historia Natural de Madrid (actuellement le Musée national des Sciences naturelles d'Espagne). Il s'agit de l'un des cabinets de curiosités les plus exceptionnels et les plus célèbres du Xviıre siècle. Romé de l'Isle, écrit dans le chapitre consacré aux Buccinites: "Soixante-huit Buccinites, du genre des Buccins à bouche échancrée, formant trente autres variétés, savoir [...] Deux de Grignon, lisses et fossiles, ainsi que tous les suivants [...] Quatre Idem de Courtagnon, conservant leur couleur fauve [...]». On reconnaitra ici sans ambiguïté des coquilles de Cryptochorda (s.str.) stromboides.

- En 1768 (Knorr 1768a, b: 30, pl. 13, fig. 3), George Wolfgang Knorr (1705-1761), graveur, illustrateur et naturaliste allemand, en donne une figure (Fig. 4C) dans ses Délices des yeux et de l'esprit, ou collection générale des différentes espèces de Coquillages que la mer renferme. Il écrit: "Cette figure représente une Voile d'artimon rougeatre. [...] La présente voile n'est ni si haute, ni si raboteuse que l'autre [figure précédente, illustrant une espèce appartenant au genre Lambis, un Strombidae], et qu'elle a aussi, proportion gardée, une coquille moins épaisse. Sa grosse babine et l'embouchure sont d'une couleur d'argent brillante». L'auteur explique dans la première partie de cet ouvrage, publiée en 1764 (Knorr 1764: 34), à propos d'une autre espèce, pourquoi il donne ce nom de "Voile d'artimon" à plusieurs de ces coquilles : "Comme cet escargot, par sa babine avancée a de la ressemblance avec cette petite voile, qui est attachée au Gouvernail des vaisseaux, ou aux mats 


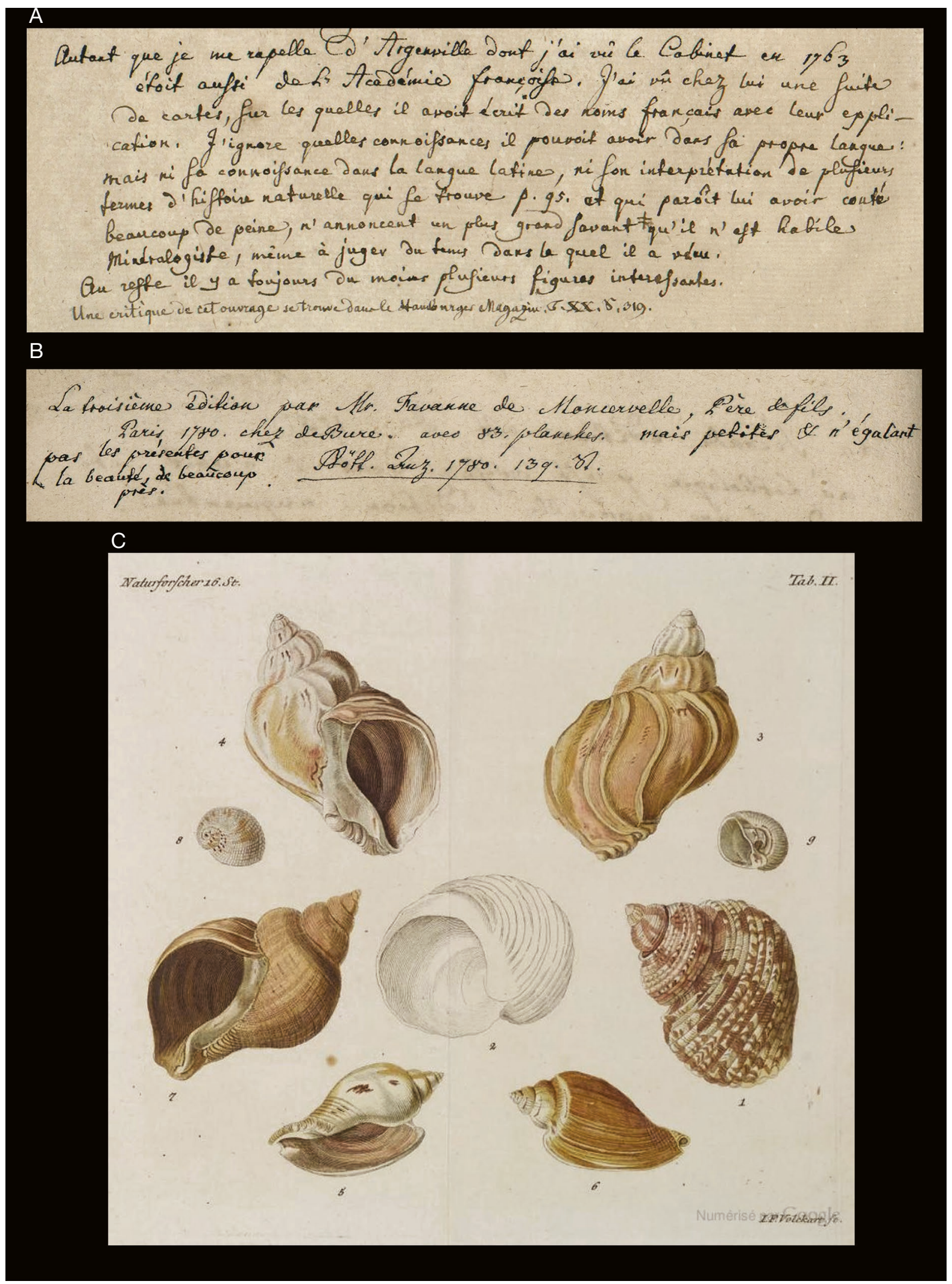

FIG. 3. - A, Notes manuscrites de Jean Hermann sur les premières pages blanches de son exemplaire de L'histoire naturelle éclaircie dans deux de ses parties principales. L'Oryctologie, qui traite des terres, des pierres, des métaux, des minéraux et autres fossiles de Dezallier d'Argenville, publié en 1755 (Bibliothèque de l'Université de Strasbourg); B, notes manuscrites de Jean Hermann sur les premières pages blanches de son exemplaire de L'histoire naturelle éclaircie dans deux de ses parties principales. La lithologie et la conchyliologie [...] de Dezallier d'Argenville, publié en 1742 (Bibliothèque de I'Université de Strasbourg); C, figure originale du Buccinum stromboides (figs 5,6) publiée par Jean Hermann en 1781 dans la revue allemande Der Naturforscher. 


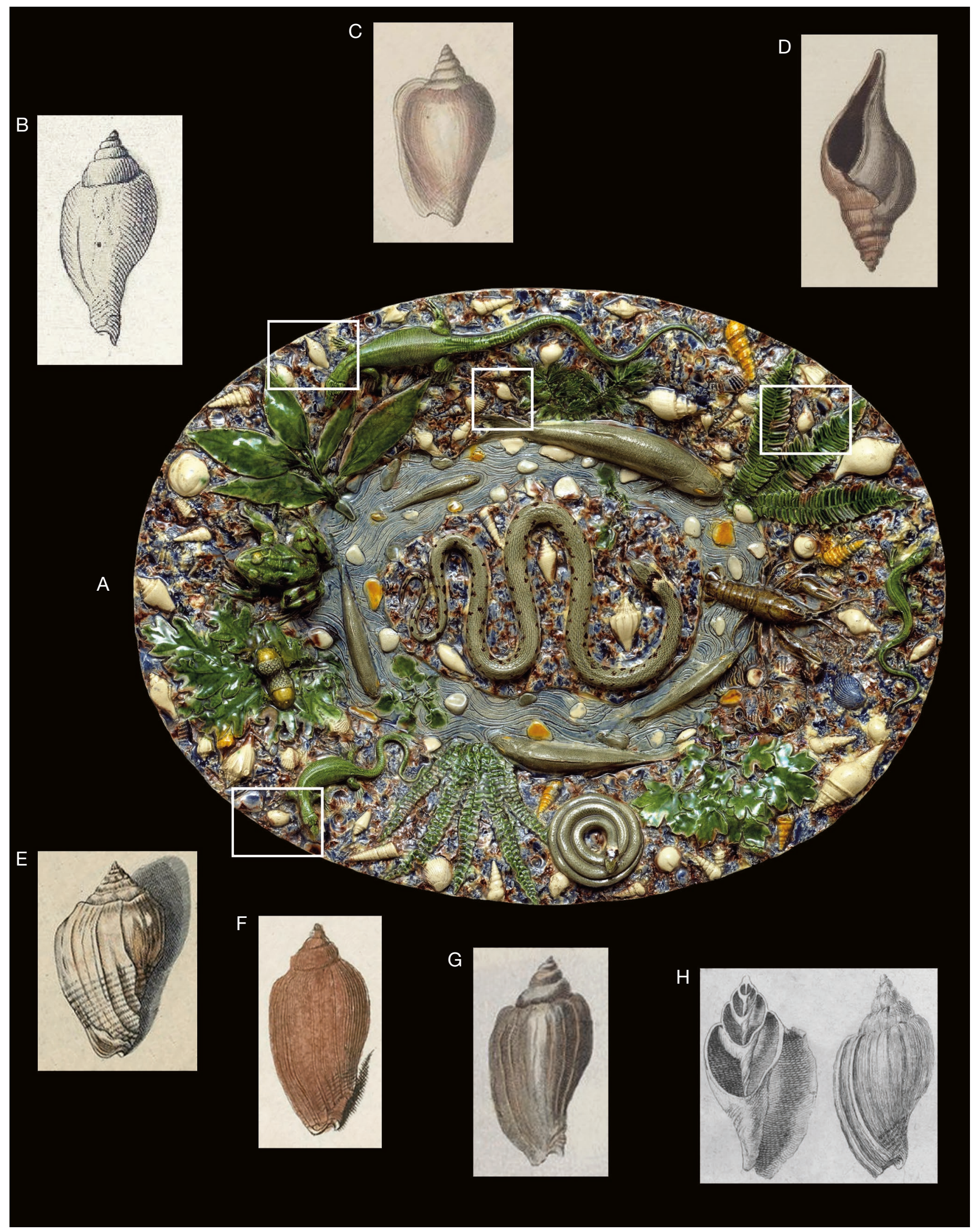

FIG. 4. - Quelques figurations anciennes de Cryptochorda (s.str.) stromboides (Hermann, 1781): A, «Rustiques figulines» post-palisséenne, pièce de vaisselle décorative, non culinaire à décor animalier comportant des moulages de coquilles dont quatre Cryptochorda (s str) stromboides (The Metropolitan Museum of Art/n $\left.{ }^{\circ} 53.225 .52\right)$. Dimensions : 52,1 x 39,7 × 7,1 cm; B, «Buccinites» in Seba (1765); C, «Voile d'artimon rougeatre » in Knorr (1768); D, «Buccinites» in Walch (1768); E, «Pyrum albidum arescens vel rugosum » in Martini (1777); F, in Favanne de Montcervelle (1780); G, in Buc'hoz (1778); H, in Schröter (1785). 


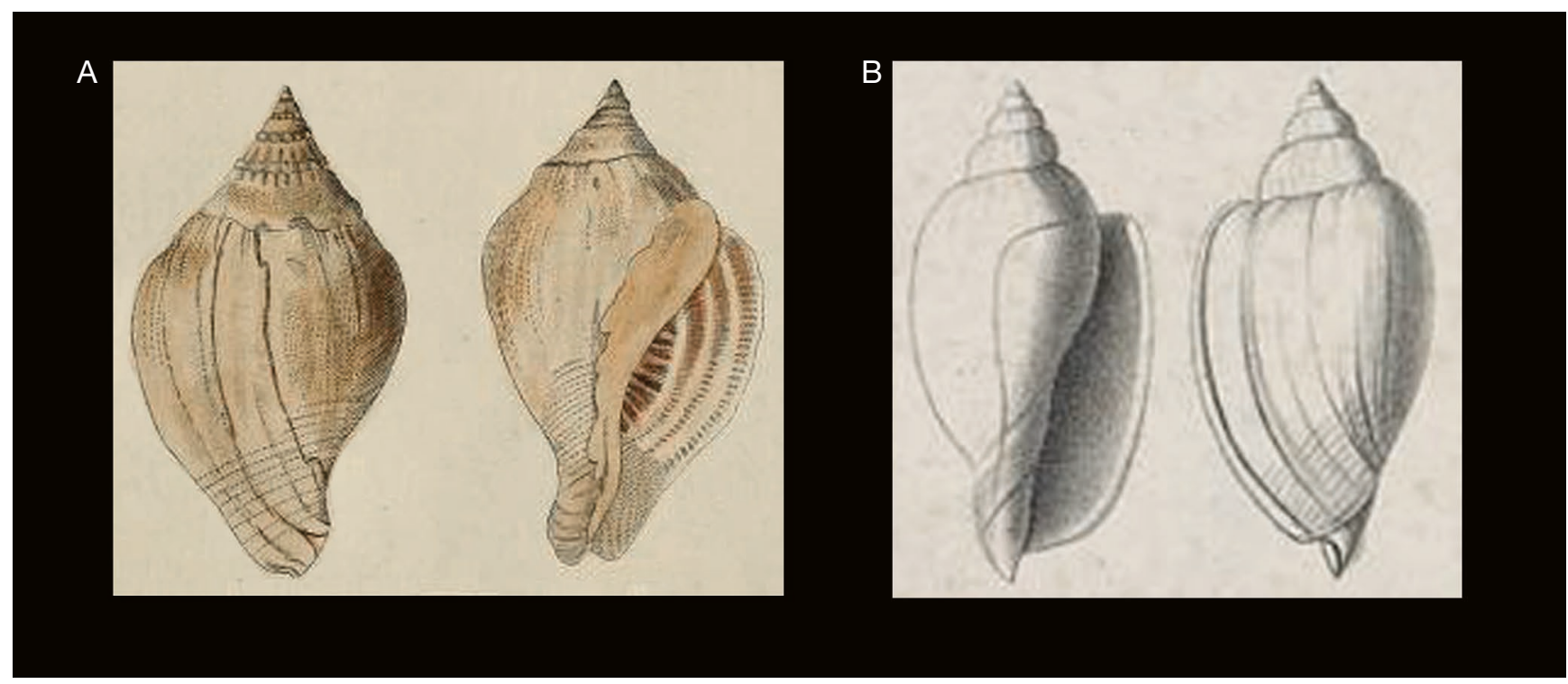

FIG. 5. - A, Sycostoma bulbus (Solander in Brander, 1766) [synonyme de Fusus bulbiformis Lamarck, 1803]. Figure originale du Pyrum arescens vel rugosum publiée par Martini (1777) dans son Neues systematisches Conchylien-Cabinet ; B, Cryptochorda (s.str.) stromboides (Hermann, 1781). Figure originale de Strombus arescens publiée par Pusch (1837) dans son Polens Paläontologie.

d'Artimon, on l'appelle Voile d'artimon, en latin Epidromis, en allemand Bezans-Segel».

- La même année, en 1768 (Walch 1768: 109, pl. C. IV, fig. 3), Jean Ernest Emmanuel Walch (1725-1778), théologien et naturaliste allemand, en donne une figure (Fig. 4D) dans son Recueil des Monumens des Catastrophes que le Globe de la Terre à éssuiées; il écrit à propos de cette espèce: «Un Buccinite lisse, dont la coquille ne semble avoir souffert qu'un changement très léger, ayant conservé même beaucoup de sa couleur naturelle. Il vient d'Angleterre» [plus probablement du bassin de Paris].

- Friedrich Wilhelm Martini (1729-1778), médecin et naturaliste allemand, en donne en 1777 (Martini 1777: 198) dans le volume 3 de son Neues systematisches Conchylien-Cabinet, un ouvrage richement illustré en couleur sur les coquillages, une figure (Fig. 4E) avec l'indication (Martini 1777: 199, fig. 32.1) : «Pyrum albidum arescens vel rugosum». Bien que l'auteur n'utilise pas le système binominal, constituant le "système linnéen", cette figure représente sans aucun doute un exemplaire de Cryptochorda (s.str.) stromboides.

- En 1778, Pierre-Joseph Buc'hoz (1731-1807), avocat, médecin botaniste de Stanislas Leszczynski (ancien roi de Pologne et duc nominal de Lorraine et de Bar), puis démonstrateur au collège royal des médecins de Nancy, publie sa première centurie de planches «représentant au naturel ce qui se trouve de plus intéressant et de plus curieux parmi les animaux [...]", imprimée sur vélin et dans laquelle nous trouvons une illustration (Buc'hoz 1778: sixième décade, pl. 6) d'une coquille de Cryptochorda (s.str.) stromboides à la figure 10 (Fig. 4G).

- Antoine-Joseph Dezallier d'Argenville (1680-1765), naturaliste, collectionneur et historien de l'art, en donne (Favanne de Montcervelle 1780: pl. 66, fig. I10) dans sa Conchyliologie, ou Histoire naturelle des coquilles de mer, d'ean donce, terrestres et fossiles, œuvre posthume publiée par Favanne de Montcervelle (1780), une bonne illustration (Fig. 4F) qui n'a pas été déterminée par Willmann \& Willmann (Carpita et al. 2009) lors de la réédition de cet ouvrage. Lamarck (1811: 76, n 7 ), Lamarck (1822: 350$\left.351, \mathrm{n}^{\circ} 7\right)$, Deshayes (1832: $\left.1145, \mathrm{n}^{\circ} 26\right)$, Deshayes (1835: 690-691, n $\left.{ }^{\circ} 12\right)$, Deshayes (1844: 417, $\left.\mathrm{n}^{\circ} 7\right)$ et Nyst (1845: $\left.590, n^{\circ} 507\right)$ identifient par erreur cette figure comme Voluta lyra Lamarck, 1803.

- Favanne de Moncervelle (1784: 490, $n^{\circ}$ 2158) dans son Catalogue systématique et raisonné, ou Description [d'un] magnifique cabinet, identifie comme "[...] Testacites fossiles de Courtagnon, dont dix Buccinites appellé le Buccin d'Émail» le spécimen de la figure I10 de la planche 66 de la troisième édition de La Conchyliologie, ou Histoire naturelle des coquilles de mer, d'eau douce, terrestres et fossiles de Dezallier d'Argenville. On reconnaitra sur cette figure une coquille de Cryptochorda (s.str.) stromboides.

- Johann Samuel Schröter (1735-1808), pasteur protestant et conchyliologiste allemand, en donne deux figures (Fig. $4 \mathrm{H}$ ) très reconnaissables (1785: 115, pl. 3, fig. 8a, b) et renvoie à l'ouvrage de Knorr publié en 1768 qui avait déjà figuré cette espèce sous le nom de "Voile d'artimon rougeatre».

- Dès 1800 Lamarck confia aux peintres Nicolas-Charles Oudinot et Nicolas Maréchal la réalisation d'une série de planches, les fameux Vélins du Muséum, figurant des mollusques fossiles du bassin de Paris récoltés par JacquesLouis-Marin Defrance, conservateur des hypothèques à Sceaux et amateur éclairé qui avait constitué une collection remarquable de fossiles provenant de Grignon [actuellement Thiverval-Grignon] (Tréguier et al. 2017). Ces vélins, un des plus précieux trésors de la Bibliothèque centrale du Muséum national d'Histoire naturelle de Paris, serviront à l'élaboration des planches du Mémoire sur les fossiles des environs de Paris. La planche 3 des vélins [non publiés à 
l'époque de Lamarck; ils ne le seront qu'en 1977 par Katherine V. W. Palmer], illustre (fig. 17a, b) un spécimen du Cryptochorda (s.str.) stromboides provenant probablement de Thiverval-Grignon (Yvelines).

Au XVIIIe siècle, les collections de curiosités se transforment en cabinets d'histoire naturelle et certains sont à l'origine de musées publics (tel que celui de la collection Jean Hermann à Strasbourg). Les coquilles circulent entre collectionneurs par le biais de ventes publiques et de catalogues de curiosités naturelles. C'est ainsi que des mollusques fossiles du bassin de Paris enrichissent les cabinets de collectionneurs allemand ou hollandais. Les inventaires se font plus raisonnés, plus structurés et les coquilles se regroupent en se référant à une systématique qui en est à ses premiers balbutiements. Cependant, certaines des figures que nous venons de citer ci-avant ne sont accompagnées que de noms vernaculaires et ne sont pas disponibles (ICZN 1999: art. 12.3). Elles montrent néanmoins que l'espèce Cryptochorda (s.str.) stromboides (Hermann, 1781), au même titre que l'Athleta (Volutospina) spinosus (Linnaeus, 1758) connue également de longue date (Pacaud \& Pons 2013), était prisée des collectionneurs de tous temps.

\section{SYSTÉMATIQUE}

Super-famille MuricoidEA Rafinesque, 1815 Famille HARPIDAE Bronn, 1849

Sous-famille CRYPTOCHORDINAE Korobkov, 1955 Genre Cryptochorda Mörch, 1858

\section{Sous-genre Cryptochorda Mörch, 1858}

Harpopsis Mayer-Eymar, 1876: 59, 60, 94. - Espèce type: Buccinum stromboides Hermann, 1781 par monotypie (invalide: synonyme objectif plus récent de Cryptochorda Mörch, 1858).

Neocryptochorda Clark, 1946: 43. — Espèce type: Cryptochorda vientoensis Clark, 1946 par désignation originale.

ESPÈCE TYPE. - Buccinum stromboides Hermann, 1781 par monotypie.

OrIGINE. - France, Éocène.

\section{REMARQUES}

Mörch (1858) introduit pour Buccinum stromboides Hermann, 1781 le genre Cryptochorda qui lui semble «avoir les plus grands rapports avec le genre Harpa (Cythara Klein), mais ses côtes sont oblitérées; comme nous avons des Scalaires sans côtes. Elle doit néanmoins constituer un sous-genre [...]". Quelques années plus tard, Mayer-Eymar (1876: 59, 60, 94) crée inutilement pour cette espèce le genre Harpopsis, pour corriger l'homonymie primaire Buccinopsis "Bayle» [nom manuscrit, porté sur une étiquette dans la collection de l'École Nationale des Mines de Paris], genre pré-employé par Conrad (1857), par Deshayes (1865) et par Jeffreys (1867). Fischer (1883: 604) a rectifié la dénomination de ce genre et a rétabli le nom introduit par Mörch.

\section{Cryptochorda (s.str.) stromboides (Hermann, 1781)}

(Figs 6A-L; 9P; 14E)

Buccinum stromboides Hermann, 1781: 53, 54, pl. 2, figs 5, 6.

«Buccinite»-Seba 1765: 126, pl. 106, fig. 22 [non disponible].

«Rothliches Bezans-segel»-Knorr 1768a: 29, pl. 13, fig. 3 [non disponible].

«Voile d'artimon rougeatre» - Knorr 1768b: 30, pl. 13, fig. 3 [non disponible].

«Buccinite lisse» - Walch 1768: 109, n 3, pl. C. IV, fig. 3 [non disponible].

«Bezaantjes of Duyfjes»-Knorr 1772: 102, pl. 13, fig. 3 [non disponible].

Pyrula arescens Martini, 1777: 199, 205 (partim), fig. 32.1 (non pl. 94, fig. 915-915a) [non disponible].

«Buccin d'Émail»-Favanne de Moncervelle 1784: 490, n 2158 [non disponible].

Voluta lyra - Lamarck 1811: 76, $\mathrm{n}^{\circ} 7 ; 1822: 350-351, \mathrm{n}^{\circ} 7$. Deshayes 1832: 1145, n⿳0 26; 1835: 690-691, n 12; 1844: 417, $\mathrm{n}^{\circ}$ 7. - Nyst 1845: 590, n 507 (non Lamarck, 1803).

Strombus arescens Pusch, 1837: 126-127, pl. 11, fig. 13a, b.

Buccinum stromboides - Schröter 1785: 115-117, pl. 3, fig. 8a, b. - Gmelin 1791: 3489, $\mathrm{n}^{\circ} 82$. - de Roissy 1805: 29-30, $\mathrm{n}^{\circ}$ 4. - Parkinson 1811: 58, pl. 5, fig. 20; 1833: 58, pl. 5, fig. 20. Defrance 1817: 111, $\mathrm{n}^{\circ}$ 1. - Dillwyn 1817: 581. - Lamarck 1822: 279, ${ }^{\circ}$ 1. - de Férussac 1822: 555. - Huot 1824: 7. - Sowerby G. B. (I) 1825: fig. 8. — Eaton 1826: 159. — Isensee 1833: 40. - Deshayes 1835: 647, ${ }^{\circ}$ 2, pl. 86, figs 9, 10 (non 8); 1844: 207-208, $\mathrm{n}^{\circ}$ 1; 1865: 495, $\mathrm{n}^{\circ}$ 1. - Anton 1838: 93, $\mathrm{n}^{\circ} 3016$. Bronn 1838: 1096, $\mathrm{n}^{\circ} 1$ (partim), pl. 41, fig. 31. - Ansted 1844: 26, fig. v. - von Geinitz 1845: 376. — Graves 1847: 629, $\mathrm{n}^{\circ} 713$ (partim). — Sowerby J. de C. in Dixon 1850: 106, pl. 7, fig. 33. d'Orbigny 1850: 320, n 420 et 369, n 635. - Lyell 1852: 329, 343, 355. - Nyst 1853: 382. — Edwards 1854: 450. - Morris 1854: 236. — Prestwich 1856: 93. - Carpenter 1861: 179. — Cornet \& Briart 1866: 174. - Winkler 1868: 49. - Dewalque 1868: 201. — von Cotta 1871: 211, fig. f. - Briart \& Cornet 1871: 28-30, pl. 2, fig. 10a-c. — Nyst in Briart \& Cornet 1874: 537. - Crocq 1880: 496. - Vasseur 1881: 244, n 41. - Delvaux 1884: 74. — Palmer 1977: 14, pl. 3, fig. 17a, b.

Buccinum strumboïdes - Lamarck 1803: 164, $\mathrm{n}^{\circ} 1$ [orthographe subséquente incorrecte].

Buccinum stromboide - Murchison et al. 1845: 286. — Pictet 1857: 44, pl. 66, fig. 30 [orthographe subséquente incorrecte].

Scaphella stromboïdes - Swainson 1840: 318, fig. 12a, b. - Sowerby G. B. (II) 1842: 253.

Harpa (Cryptochorda) stromboides - Mörch 1858: 43-44.

Harpa (Harpopsis) stromboides - Mayer-Eymar 1876: 60.

Harpopsis stromboides - Briart \& Cornet 1877: 24. — Tryon 1883: 178, pl. 54, fig. 34. - Zittel 1885: 282-283, fig. 400. - MayerEymar 1886: 255. — Newton 1891: 104. — Koken 1896: 144, fig. 132.1. - Fritel 1903: 213-214, n 427, pl. 19, fig. 9.

Cryptochorda stromboides - Fischer 1883: 604-605. — Cossmann 1889: 192-193 (partim); 1899: 76-78, pl. 4, figs 1; 4; 1896: 233- 


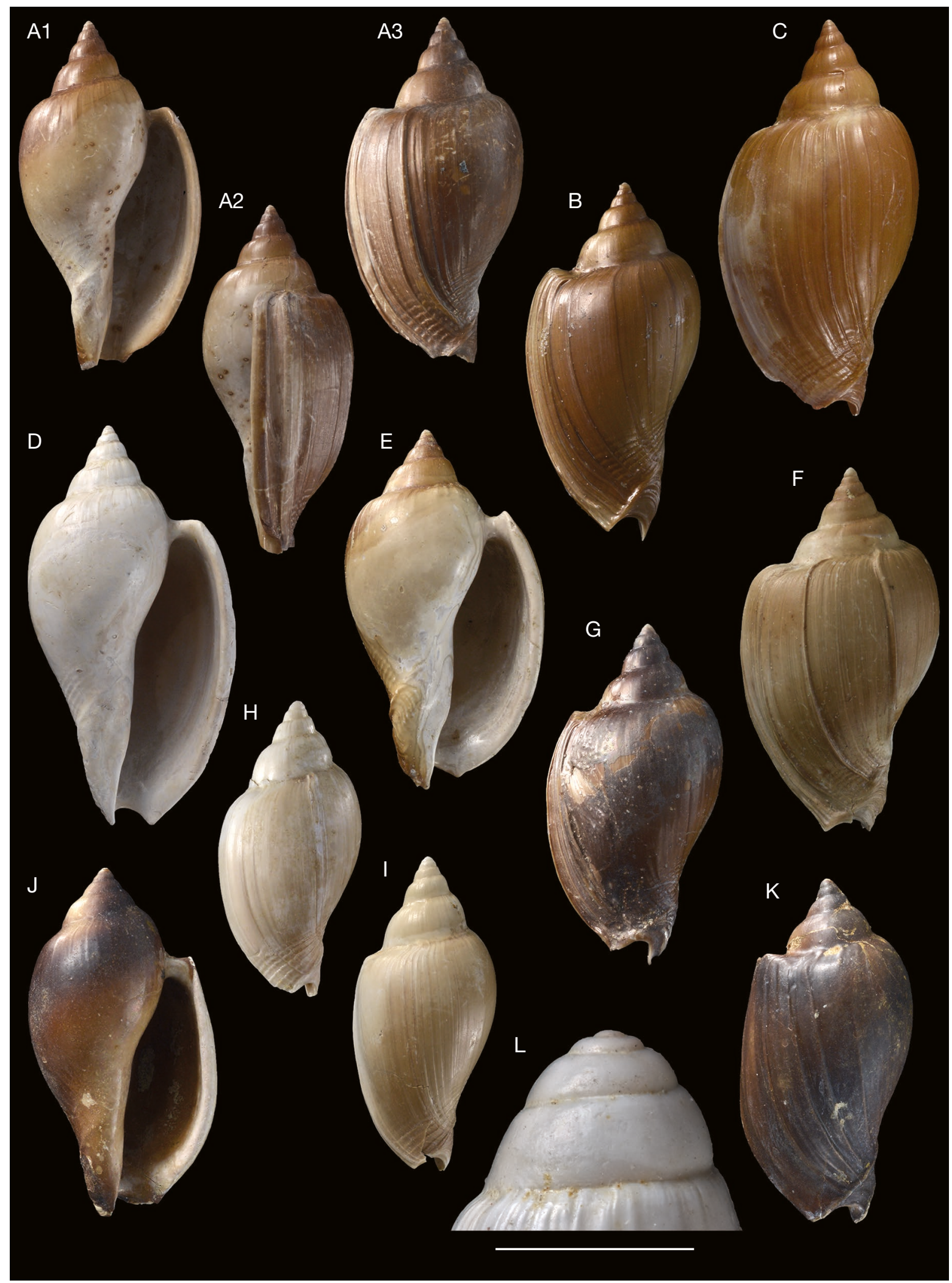

FiG. 6. - A-L, Cryptochorda (s.str.) stromboides (Hermann, 1781). A, Néotype MNHN.F.A57573 (coll. de Roissy), Lutétien de Courtagnon (Marne), H. : 55,7 mm; B, MNHN.FA71408 (coll. Pacaud), Lutétien de Damery (Marne), H. : 46,7 mm; C, MNHN.F.A71409 (coll. Staadt), Lutétien de Damery (Marne), H. : 51,6 mm; D, MNHN.F.A71410 (coll. d'Orbigny), Lutétien de Thiverval-Grignon (Yvelines), H. : 61,2 mm; E, MNHN.F.A71411 (coll. Pacaud), Lutétien de Damery (Marne), H. : 48,0 mm; F, MNHN.F.A71412 (coll. Pacaud), Lutétien de Damery (Marne), H. : 55,5 mm; G, MNHN.F.A71413 (coll. Faullummel), Auversien (Bartonien) de Mécringes (Marne), H. : 48,0 mm; H, spécimen juvénile, MNHN.F.A71414 (coll. Staadt), Lutétien de Damery (Marne), H. : 27,7 mm; I, spécimen juvénile, MNHN.F.A71415 (coll. Staadt), Lutétien de Damery (Marne), H. : 34,8 mm; J, MNHN.F.A71416 (coll. Faullummel), Auversien (Bartonien) de Dhuisy (Seine-et-Marne), H. : 36,9 mm; K, MNHN.F.A71417 (coll. Faullummel), Auversien (Bartonien) de Dhuisy (Seine-et-Marne), H. : 39,5 mm; L, MNHN.F.A71418 (coll. Pacaud), Lutétien de Fontenayen-Vexin, Bois du But (Eure), protoconque. Échelle: L, $2 \mathrm{~mm}$. 
234, pl. 9, fig. 3; 1913: 196 - Bernard 1895: 502, fig. 258B. Bureau 1900: 386. - Chédeville 1904: 419. - Pezant 1910: 196. — Cossmann \& Pissarro 1911: pl. 42, fig. 203-1. — Hedley \& Pilsbry 1912: 47. — Glibert 1933: 97-98, pl. 6, fig. 3; 1938: 114, pl. 4, fig. 5; 1960: 48 (partim); 1985: 336-337. — Wrigley 1934: 13. - Morley Davies 1935: 305, fig. 463. — Palmer 1937: 400. Furon \& Soyer 1947: 122, 165, pl. 16, fig. 203-1. - Korobkov 1955: 337, fig. 167 , pl. 84, figs 19, 20. - Termier \& Termier 1960: 366, fig. 2694. - Strausz 1966: 135. - Chavan \& Montocchio 1968: 141, fig. 234. - Pomerol \& Feugueur 1974: pl. 12, fig. 11. - Fischer 1980: 320 (partim), pl. 155, fig. 5; 2000: 346 (partim), pl. 171, fig. 5. — Brébion 1982: 132. — Geys \& Marquet 1983: 118, pl. 50, fig. 2. — Bone \& Bone 1985: pl. 4, fig. 17. Merle 1989: 97. — Le Renard \& Pacaud 1995: 118. — Pacaud \& Le Renard 1995: 167. — Tracey et al. 1996: 121, 123. — Merle \& Pacaud 2003: 74, fig. 7a. — Merle et al. 2008: 208. — Pacaud 2008: 64. - Courville et al. 2012: pl. 8, figs 14-16 et 18, 19. - Dulai et al. 2017: 158, figs 64-65.

Buccinum (Harpopsis, Buccinopsis) stromboides - Fritel 1886: pl. 3, fig. 31 .

Buccinum (Cryptochorda) stromboides-Meunier 1912: 298, fig. 111.

Cryptochorda (s.str.) stromboides - Wenz 1943: 1323, fig. 3761.

MATÉRIEl TYPE. - Néotype MNHN.F.A57573 (coll. de Roissy) désigné ici. Malgré nos recherches, le matériel type du Lutétien (Éocène moyen) de Courtagnon (Marne) n’a pas été retrouvé (voir ci-avant). La désignation d'un néotype s'impose pour clarifier le statut taxonomique de cette espèce, syntopique dans certains gisements de Cryptochorda (s.str.) altavesna n. sp. décrite ci-après et confondue jusqu'ici avec C. (s.str.) stromboides. Nous avons choisi un spécimen, cohérent avec le type porte-nom perdu, provenant de la localité type originale de Courtagnon (Marne). Notre démarche respecte les règles requises par l'ICZN (1999: art. 75) en ce qui concerne la désignation d'un néotype.

LOCALITÉ TYPE. - Courtagnon (Marne), Lutétien (Éocène moyen).

Dimensions. - (Néotype) hauteur: 55,7 mm; diamètre: 29,0 mm.

Autre matériel eXaminé. - Voir Annexe 1.

\section{DESCRIPTION}

La coquille est grande, épaisse, ovale-oblongue et un peu ventrue, ayant un angle apical de $65^{\circ}$. La spire est courte, conique et présente une protoconque de 3 tours $1 / 2$ et une téléoconque de 5 tours peu convexes, relativement plans, lisses et séparés par une suture superficielle presque entièrement recouverte par le vernis. La protoconque est petite, légèrement globuleuse, à petit nucléus et aux tours convexes et lisses. La transition protoconque/téléoconque est indistincte, mais nettement visible chez les spécimens montrant une coloration brune préservée; la protoconque restant blanche. Le dernier tour est très grand et occupe $80 \%$ de la hauteur totale; il se termine par un cou relativement court et muni d'une fasciole siphonale, large, circonscrite par deux fortes crêtes. Le dernier tour est ventru dans sa partie adapicale, atténué au niveau du cou et orné dans cette région d'environ 8 stries décurrentes très serrées. L'ensemble de la téléoconque est lisse, porcelané et dépourvu de sculpture, on observe seulement de fortes stries d'accroissement prosocyrtes, au contour parasigmoidal, surtout visibles sur les premiers tours. Le dernier tour est également marqué, près du labre, par de forts arrêts de croissance irréguliers. L'ouverture est oblongue, large dans sa partie médiane, étroite en avant et profondément échancrée, présentant une large gouttière, entaillant l'angle adapical. Le canal siphonal est court et étroit. L'encoche siphonale est large et profonde. La columelle est légèrement convexe dans la zone pariétale, à peine excavée dans sa partie médiane et légèrement infléchie et rectiligne vers le cou. Elle est lisse et ne montre aucune trace de plis. Le bord de l'ouverture présente une callosité columellaire, d'une couleur blanchâtre, très largement étalée latéralement, envahissant la base du pénultième tour et, se pliant alors brusquement en arrière, se dirige parallèlement à la suture et produit une bande spirale étroite sur la partie abapicale des tours. Cette callosité columellaire longe également la columelle jusqu'au canal siphonal et la fasciole. Ce callus, bien appliqué, est peu épais et recouvre à peine les stries décurrentes et les crêtes postérieure et antérieure de la fasciole siphonale. Le labre est épais, de direction orthocline, au contour régulièrement convexe en vue aperturale sur les $3 / 4$ de sa longueur, puis montre une légère inflexion dans la zone abapicale. Il est fortement sinueux en son extrémité, au niveau de la gouttière entaillant l'angle adapical, il se termine près de l'échancrure siphonale, descendant moins bas que la pointe aiguë de la columelle. L'intérieur de l'ouverture et le bord interne du labre sont lisses et vernissés. La coquille conserve très souvent sa couleur d'origine, une teinte uniforme allant d'un brun clair, ambrée à une teinte brun-rougeâtre plus ou moins intense. L'exposition sous lumière UV ne montre aucune trace de motif coloré résiduel.

\section{REMARQUES}

Les coquilles récoltées dans les sables auversiens (Bartonien, Éocène moyen) présentent souvent une teinte uniforme brunrougeâtre plus intense que celle des exemplaires de $C$. (s.str.) stromboides récoltés au Lutétien (Fig. 6G, J, K). Ces spécimens ne présentent néanmoins aucun caractère permettant de les en séparer. Les rares spécimens (MNHN.F.A57655) provenant du Bartonien de Loire-Atlantique sont également indiscernables de C. (s.str.) stromboides. L'espèce est signalée à l'Éocène de l'Oural en Russie (Murchison et al. 1845: 286; Eichwald 1853: 418) mais nous n'avons pu examiner aucun spécimen provenant de cette région et l'espèce n'est d'ailleurs plus citée dans le travail d'Alexeev (1963). Quant aux assertions de Cornet \& Briart (1866: 174), de Dewalque (1865: $721 ; 1866: 71)$, de Ortlieb \& Chellonneix (1870: 121) et de Briart \& Cornet (1871: 28-30, pl. 2, fig. 10a-c; 1877: 24), sur la présence de cette espèce dans les sables du Danien (Paléocène inférieur) de Mons (Belgique), Glibert (1985: 336), au cours de sa révision des gastéropodes du Calcaire de Mons, insiste bien sur le fait qu'il n'a jamais vu aucun spécimen décrit sous le nom de Buccinum stromboides et pense qu'il s'agit probablement d'une erreur de récolte ou d'étiquetage parce que la coquille est décrite comme brillante, tandis que tous les fossiles du Danien de Mons sont toujours mats.

Le taxon Buccinum stromboides Hermann, 1781 a été discuté par Pusch (1837: 126-127, pl. 11, fig. 13a, b) sous le nom de Strombus arescens, en l'identifiant à une espèce décrite antérieurement par Martini (1777), qui n'en précisait pas cependant l'origine mais qui provenait probablement de Courtagnon 


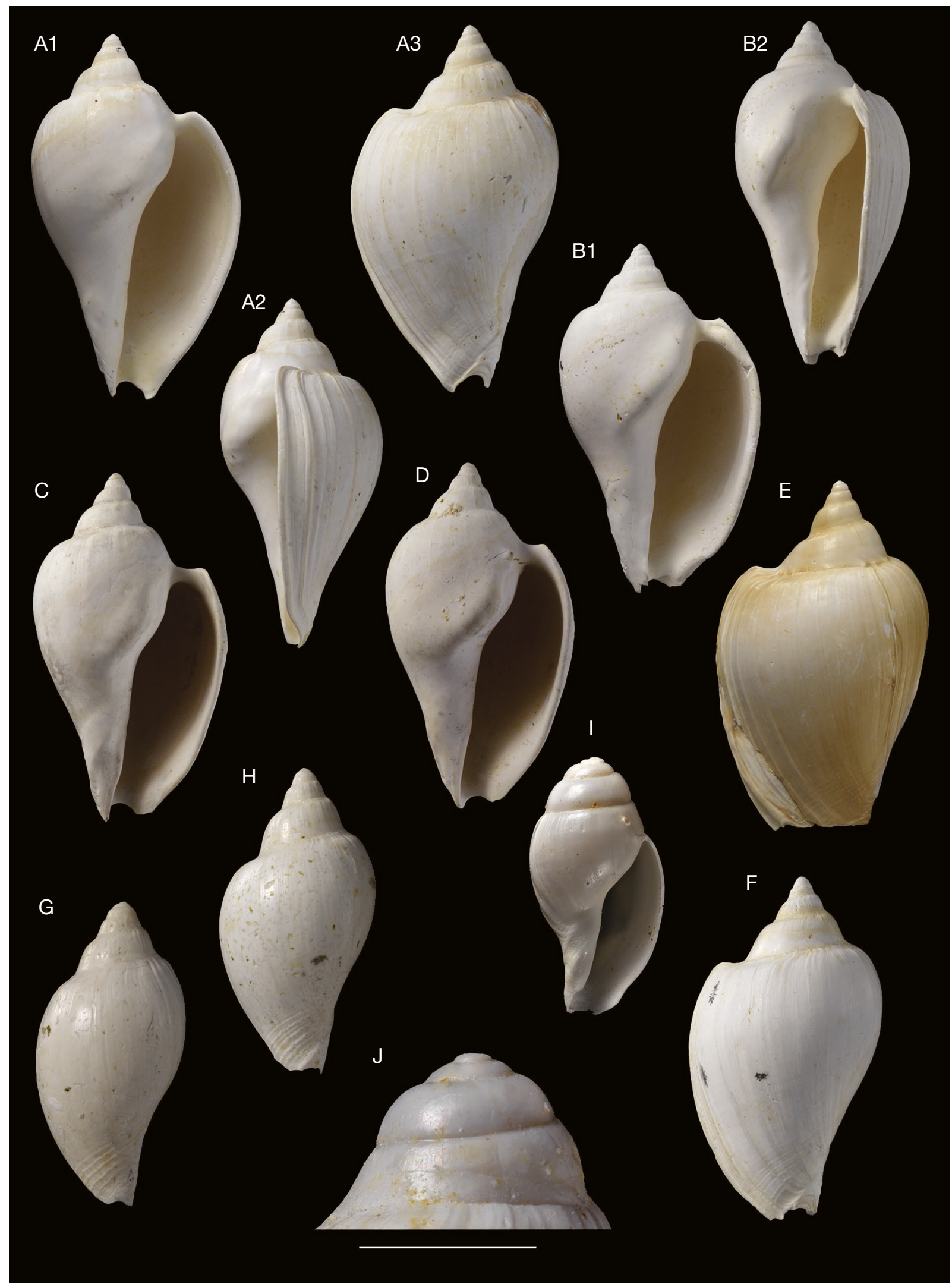

Fig. 7. - A-J, Cryptochorda (s.str.) altavesna n. sp. A, B, F-H, Lutétien de Authevernes (Eure); C, D, Lutétien de Parnes (Oise); E, Lutétien de Maulette (Yvelines); I, J, Lutétien de Fontenay-en-Vexin, Bois du But (Eure). A, Holotype MNHN.F.A71419 (coll. Sautereau), H. : 44.8 mm; B, paratype MNHN.F.A71420 (coll. Sautereau), H. : 42,8 mm; C, MNHN.F.A71421 (coll. de Roissy), H. : 43,1 mm; D, MNHN.F.A71422 (coll. de Roissy), H. : 40,2 mm; E, MNHN.F.A71428 (coll. Sautereau), H. : 40,8 mm; F, paratype MNHN.F.A71423 (coll. Sautereau), H. : 46,0 mm; G, spécimen juvénile, paratype MNHN.F.A71424 (coll. Sautereau), H. : 22,2 mm; H, spécimen juvénile, paratype MNHN.F.A71425 (coll. Sautereau), H. : 26,4 mm; I, MNHN.F.A71440 (coll. Pacaud), H. : 7,1 mm; J, MNHN.F.A74441 (coll. Pacaud), protoconque. Échelle: J, $2 \mathrm{~mm}$. 


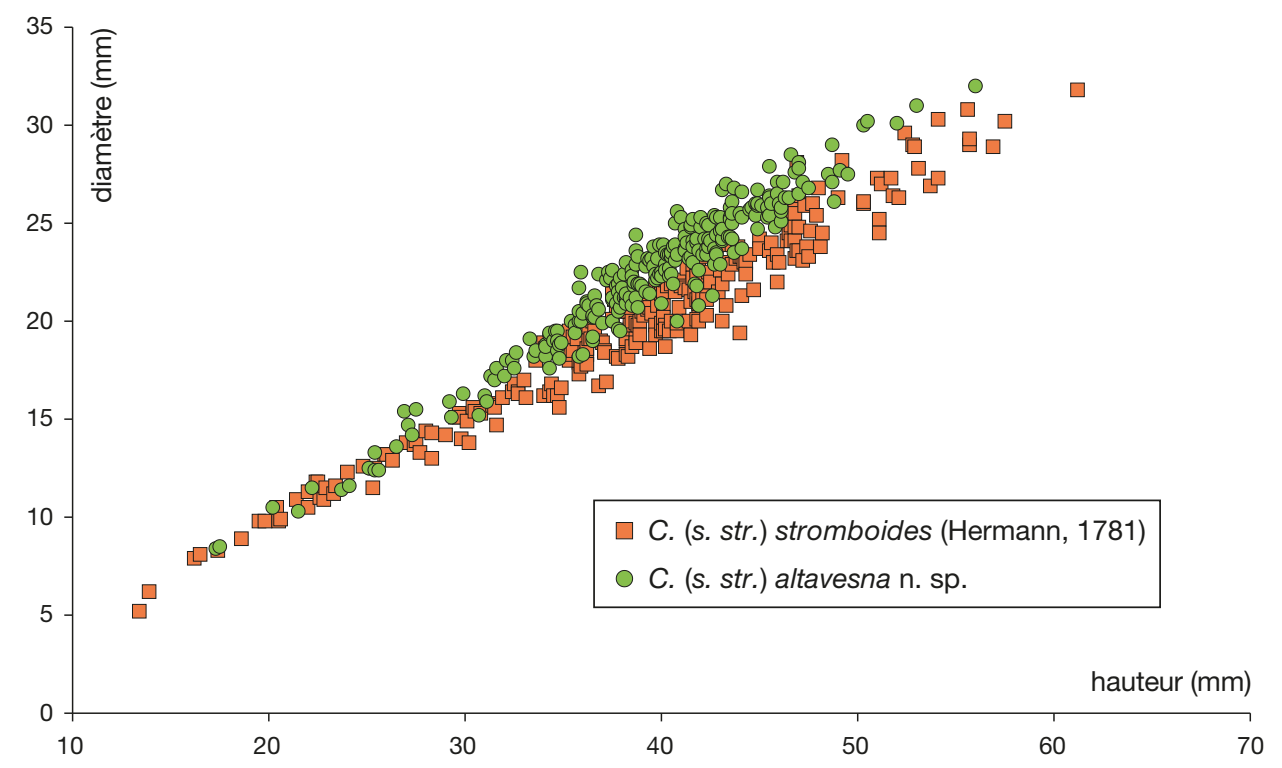

FIG. 8. - Diagramme de dispersion du rapport entre hauteur et diamètre maximal des espèces Cryptochorda (s.str.) stromboides (Hermann, 1781) ( $\square$ ) et Cryptochorda (s.str.) altavesna n. sp. (0).

(Marne), comme la plupart des coquilles fossiles lutétiennes récoltées dans le bassin de Paris à cette époque. Martini (1777: 205, fig. 32.1, pl. 94, fig. 915-915a) a en effet décrit une espèce sous le nom de Pyrum arescens ou "Poire ridée à clavicule un peu noueuse» et a traité son nom comme valide, mais dans son travail le principe de la nomenclature binominale n'est pas appliqué de façon cohérente, de sorte que ce nom n'est pas disponible (Opinion 184 [1944]). Pusch a employé pour un taxon le nom de Strombus arescens, tout en citant dans la synonymie de cette espèce le nom Harpa [en fait Pyrum] arescens, qu'il a attribué (p. 127) à Martini en l'accompagnant d'une référence bibliographique et en renvoyant à la figure 915 de la planche 94. Par cet acte, Pusch a rendu disponible le nom Strombus arescens Pusch, 1837 en accord avec l'article 11.5.2 de l'ICZN (1999). Cependant, Martini donnait deux interprétations différentes de son Pyrum arescens ainsi qu'en témoigne ces illustrations. La coquille illustrée à la figure 32.1 (Fig. 4E) (Martini 1777: 198) du Neues systematisches Conchylien-Cabinet, avec l'indication (Martini 1777: 199) : "Pyrum albidum arescens vel rugosum» représente sans aucun doute un exemplaire adulte de Cryptochorda (s.str.) stromboides. On y distingue, sur le dernier tour, près du labre, les forts arrêts de croissance irréguliers caractéristiques de l'espèce. Pour autant, la coquille illustrée planche 94 (fig. 915-915a) (Fig. 5A) du même ouvrage, avec l'indication (Martini 1777: 205) : «Pyrum arescens vel rugosum» ne représente pas du tout la même espèce mais un morphe à spire allongée du Sycostoma bulbus (Solander in Brander, 1766) [synonyme de Fusus bulbiformis Lamarck, 1803] de l'Éocène du Bassin Anglo-Parisien (Solander in Brander 1766: 27, pl. 4, fig. 54 ; Courville et al. 2012: pl. 6, fig. 35). Par ailleurs, Gmelin (1791: 3485, $\mathrm{n}^{\circ}$ 59) introduit pour la coquille de la planche 94 de Martini le nom Buccinum umbilicatum (Schreibers 1793: 150, n 42; Dillwyn 1817: 617, n 71; Pfeiffer 1840: 25; Anton 1838: 79). Ce nom est toutefois un synonyme subjectif plus récent de Murex bulbus
Solander in Brander, 1766. Il convient enfin de remarquer que Pusch (1837: pl. 11, fig. 13a, b) figure bien (Fig. 5B) sous le nom de Strombus arescens une coquille de Cryptochorda (s.str.) stromboides. Il cite même le nom Buccinum stromboides Hermann, 1781 en synonymie, constatant qu'il s'agit d'un nom plus récent que celui introduit par Martini en 1777; considération au demeurant tout-à-fait justifiée [voir discussion ci-avant sur l'indisponibilté des noms proposés par Martini \& Chemnitz]. Cependant il renvoie non pas à la figure 32.1 de Martini mais à la figure 915 de la planche 94, qui représente un Sycostoma.

Cryptochorda (s.str.) altavesna n. sp. (Figs 7A-J ; 9A-O; 14A, F)

urn:Isid:zoobank.org:act:030FCBAB-8568-4EDA-87E1-33BC0F5A8AF9

Buccinum stromboides variété a - Deshayes 1835: 647, pl. 86, fig. 8 (non 9-10).

Buccinum stromboides - Deshayes 1853 (non Hermann, 1781): 71 (partim), pl. 118, fig. 11.

Buccinum stromboides 2e variété - Deshayes 1865: 495.

MATÉRIEL TYPE. — Holotype: MNHN.F.A71419 (coll. Sautereau); paratypes : 11 ex., MNHN.F.A71420, A71423, A71424, A71425, A71426, A71427, A71432 et A71433 (coll. Sautereau).

LOCALITÉ TYPE. - Authevernes (Eure, France), Lutétien (Éocène moyen).

ÉTYMologie. - De sa localité type dont la forme en Altavesna, «Haute avesne» (Haute pâture), est documenté en 1051-1056 (de Beaurepaire 1981). Nom donné en apposition.

DiMENSIONS. - (Holotype) hauteur: 44,8 mm; diamètre: 26,0 mm.

Autre matériel examiné. - Voir Annexe 2. 


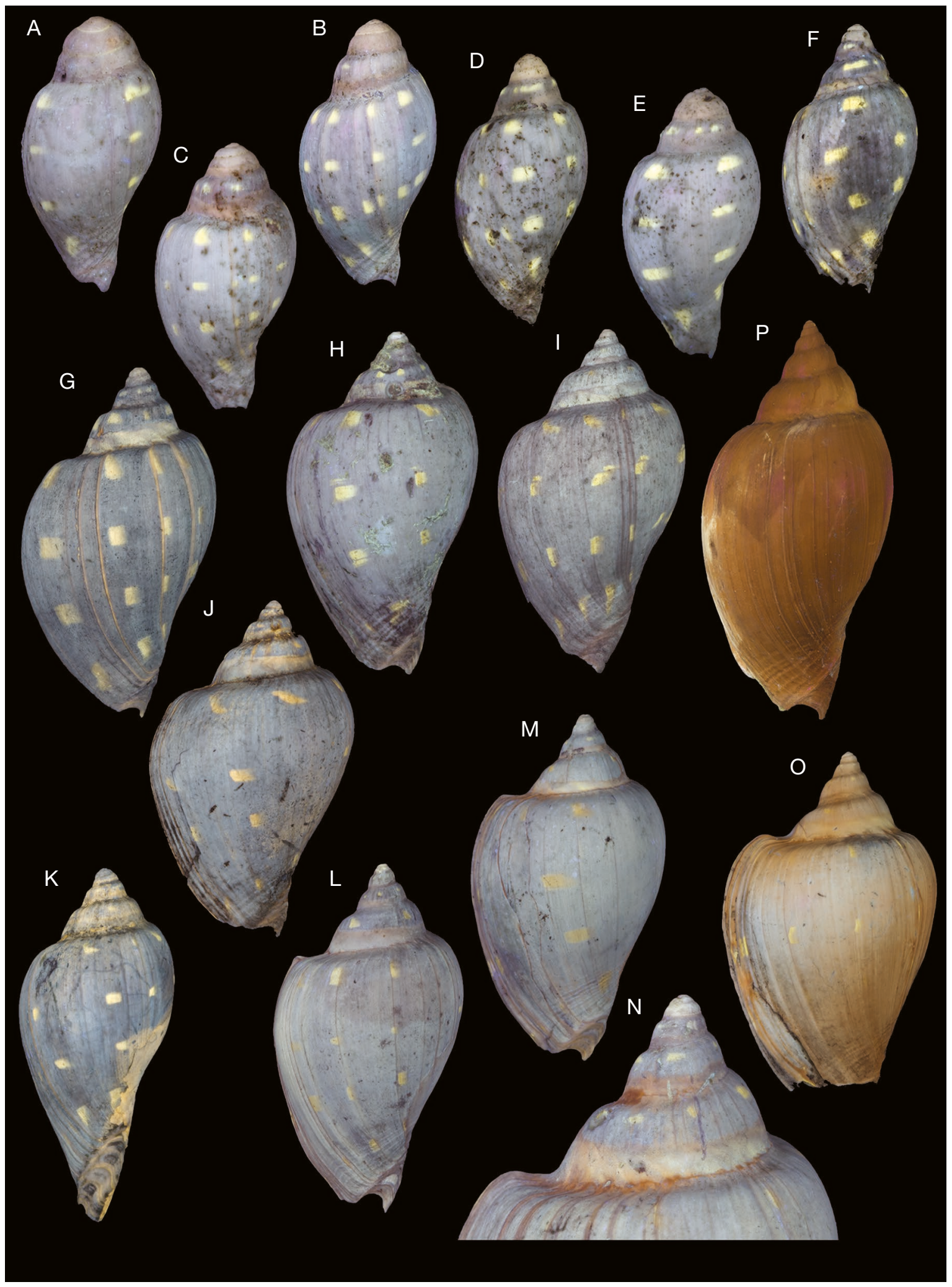

FiG. 9. - Motifs colorés résiduels révélés sous lumière UV sur des spécimens juvéniles et adultes. A-O, Cryptochorda (s.str.) altavesna n. sp. A-F, Lutétien de Fontenay-en-Vexin, Bois du But (Eure); G, I, K-M, Lutétien de Parnes (Oise); H, J, N, Lutétien de Authevernes (Eure); O, Lutétien de Maulette (Yvelines); P, Cryptochorda (s.str.) stromboides (Hermann, 1781), Lutétien de Damery (Marne). A, MNHN.F.A71434 (coll. Pacaud), H. : 6,8 mm; B, MNHN.F.A71435 (coll. Pacaud), H. : 9,4 mm; C, MNHN.F.A71436 (coll. Pacaud), H. : 10,7 mm; D, MNHN.F.A71437 (coll. Pacaud), H. : 16,2 mm; E, MNHN.F.A71438 (coll. Pacaud), H. : 10,3 mm; F, MNHN.F.A71439 (coll. Pacaud), H. : 18,5 mm; G, MNHN.F.A71429 (coll. de Roissy), H. : 32,7 mm; H, Paratype MNHN.F.A71426 (coll. Sautereau), H. : 32,3 mm; I, MNHN.F.A71430 (coll. de Roissy), H. : 36,6 mm; J, Paratype MNHN.F.A71427 (coll. Sautereau), H. : 42,5 mm; K, MNHN.F.A71431 (coll. de Roissy), H. : 28,9 mm; L, MNHN.F.A71421 (coll. de Roissy), H. : 43,1 mm; M, MNHN.F.A71422 (coll. de Roissy), H. : 40,2 mm; N, Paratype MNHN.F.A71432 (coll. Sautereau), H. : 42,1 mm; O, MNHN.F.A71428 (coll. Sautereau), H. : 40,8 mm; P, MNHN.F.A71409 (coll. Staadt), H. : 51,6 mm. 


\section{DESCRIPTION}

La coquille est grande, épaisse, pyriforme, ventrue en arrière, ayant un angle apical de $75^{\circ}$. La spire est courte, conique et présente une protoconque de 3 tours $1 / 2$ et une téléoconque de 4 tours peu convexes, lisses, relativement plans, pourvus d'une large rampe suturale, légèrement canaliculée et séparés par une suture superficielle presque entièrement recouverte par le vernis. La protoconque est large, globuleuse, à petit nucléus et aux tours convexes et lisses. La transition protoconque/téléoconque est indistincte. Le dernier tour est très grand et occupe $84 \%$ de la hauteur totale; il se termine par un cou relativement court et muni d'une fasciole siphonale, large, circonscrite par deux fortes crêtes. Le dernier tour est ventru dans sa partie adapicale, atténué au niveau du cou et orné dans cette région d'environ 12 stries décurrentes, très serrées. L'ensemble de la téléoconque est lisse, porcelanée et dépourvue de sculpture, on observe seulement de fortes stries d'accroissement prosocyrtes, au contour parasigmoidal, surtout visibles près de la suture, sur la rampe suturale. Le dernier tour est également marqué, près du labre, par de forts arrêts de croissance irréguliers. L'ouverture est oblongue, ample, large dans sa partie médiane, étroite en avant et profondément échancrée, présentant une large gouttière entaillant l'angle adapical, au niveau de la rampe suturale. Le canal siphonal est court et étroit. L'encoche siphonale est large et profonde. La columelle est très convexe dans la zone pariétale, en arrière du bourrelet calleux, fortement excavée dans sa partie médiane et légèrement infléchi et rectiligne vers le cou où elle est comprimée dorso-ventralement. Elle est lisse et ne montre aucune trace de plis. Le bord de l'ouverture présente une callosité columellaire, d'une couleur blanchâtre, très largement étalée latéralement, envahissant la base du pénultième tour et, se pliant alors brusquement en arrière, se dirige parallèlement à la suture et produit une bande spirale étroite sur la partie abapicale des tours. Cette callosité columellaire longe également la columelle jusqu'au canal siphonal et la fasciole. Ce callus, bien appliqué, est épais et recouvre les stries décurrentes et le bourrelet postérieur de la fasciole siphonale. La face ventrale présente, dans sa partie adapicale et un peu en retrait de la partie postérieure de la columelle, un bourrelet calleux, très épais, oblique et sinueux, incliné vers l'ouverture. Le labre est épais, de direction orthocline, au contour régulièrement convexe en vue aperturale, fortement sinueux en son extrémité, au niveau de la gouttière entaillant l'angle adapical. Il se termine près de l'échancrure siphonale, descendant plus bas que la pointe aiguë de la columelle. L'intérieur de l'ouverture et le bord interne du labre sont lisses et vernissés. Ordinairement blanche, la coquille conserve très rarement sa couleur d'origine, une teinte uniforme d'un brun très clair. Par ailleurs, elle présente également un motif résiduel de couleur, observé seulement sous éclairage UV. Il est constitué de quatre alignements spiraux de taches claires et fluorescentes sur un fond sombre. Ces taches sont larges et de forme quadrangulaire et elles sont alignées axialement. Ces alignements sont régulièrement espacés: un premier est situé près de la rampe adapicale; deux autres sont situés sur la partie médiane du dernier tour et, enfin, la dernière se trouve sur la partie abapicale, sur le cou, au niveau des stries décurrentes. Le bourrelet calleux, incliné vers l'ouverture, est clair et lumineux. L'intérieur de l'ouverture est très clair et fluorescent.

\section{Discussion}

Cette espèce est nettement différente de Cryptochorda (s.str.) stromboides. Cependant, même s'il s'agit d'une forme de morphologie générale comparable, aucun auteur n'a séparé les deux espèces et seul Deshayes (1835: 647, pl. 86, fig. 8) en a donné les caractères : "Notre première variété est particulièrement remarquable par une grosse callosité oblique, inclinée vers l'ouverture et située sur la partie supérieure de la columelle» et en a fait une variété (non dénommée). C'est elle qui est illustrée dans son Traité élémentaire de Conchyliologie (Deshayes 1853: pl. 118, fig. 11) (Fig. 14F) et non l'espèce décrite par Hermann. Par ailleurs, et à titre anecdotique, on soulignera également que dans sa collection [lot $\mathrm{n}^{\circ}$ 15029], Alexandre Brongniart avait étiqueté "Buccinum stromboides?"(Fig. 14A-C), un spécimen (MNHN.F.A57647) de cette espèce provenant du Lutétien (Éocène moyen) de Parnes (Oise), relevant ainsi un doute bien légitime; ses autres spécimens (MNHN.F.A57590 et A57591) [lot $\mathrm{n}^{\circ} 15028$ ] sont déterminés sans ambiguïté "Buccinum stromboides» (Fig. 14D-E) et comparés au Buccinum laevissimum [synonyme de Bullia laevissima (Gmelin in Linnaeus, 1790)]. Cryptochorda (s.str.) altavesna n. sp. se caractérise évidement par la présence sur la face ventrale, même sur les spécimens juvéniles, d'un bourrelet calleux, très épais, sinueux, incliné vers l'ouverture, totalement absent chez $C$. (s.str.) stromboides et par son galbe pyriforme, très élargi en arrière. On soulignera également que la coquille présente 4 tours et non 5, munis d'une large rampe suturale, que la protoconque est plus large, plus globuleuse, aux tours moins hauts et que la columelle est comprimée dorso-ventralement dans la zone abapicale de l'ouverture. L'ensemble de la téléoconque est lisse mais est ornée d'une douzaine de stries décurrentes, très serrées et non 8. La columelle est nettement plus convexe dans la zone pariétale et plus fortement excavée dans sa partie médiane et rectiligne vers le cou. Le labre montre un contour régulièrement convexe en vue aperturale, sans la légère inflexion dans la zone abapicale qui caractérise $C$. (s.str.) stromboides; il se termine près de l'échancrure siphonale, descendant au-delà de la pointe aiguë de la columelle et non moins bas que celle-ci. Par ailleurs, les résultats de l'analyse biomètrique justifient la distinction de ces deux espèces (Fig. 8). Enfin, au lieu d'avoir seulement une teinte brune plus ou moins intense, la coquille montre, non seulement une teinte uniforme d'un brun très clair (sur de rares exemplaires: Figs 7E; 9O) mais aussi, sous éclairage UV, un motif résiduel de couleur constitué de quatre alignements spiraux de taches claires et fluorescentes sur un fond sombre (Fig. 9A-O). Ce motif est déjà présent et observable, sur quatre alignements spiraux, sur des exemplaires juvéniles d'à peine $4 \mathrm{~mm}$ (Fig. 9A-F). 


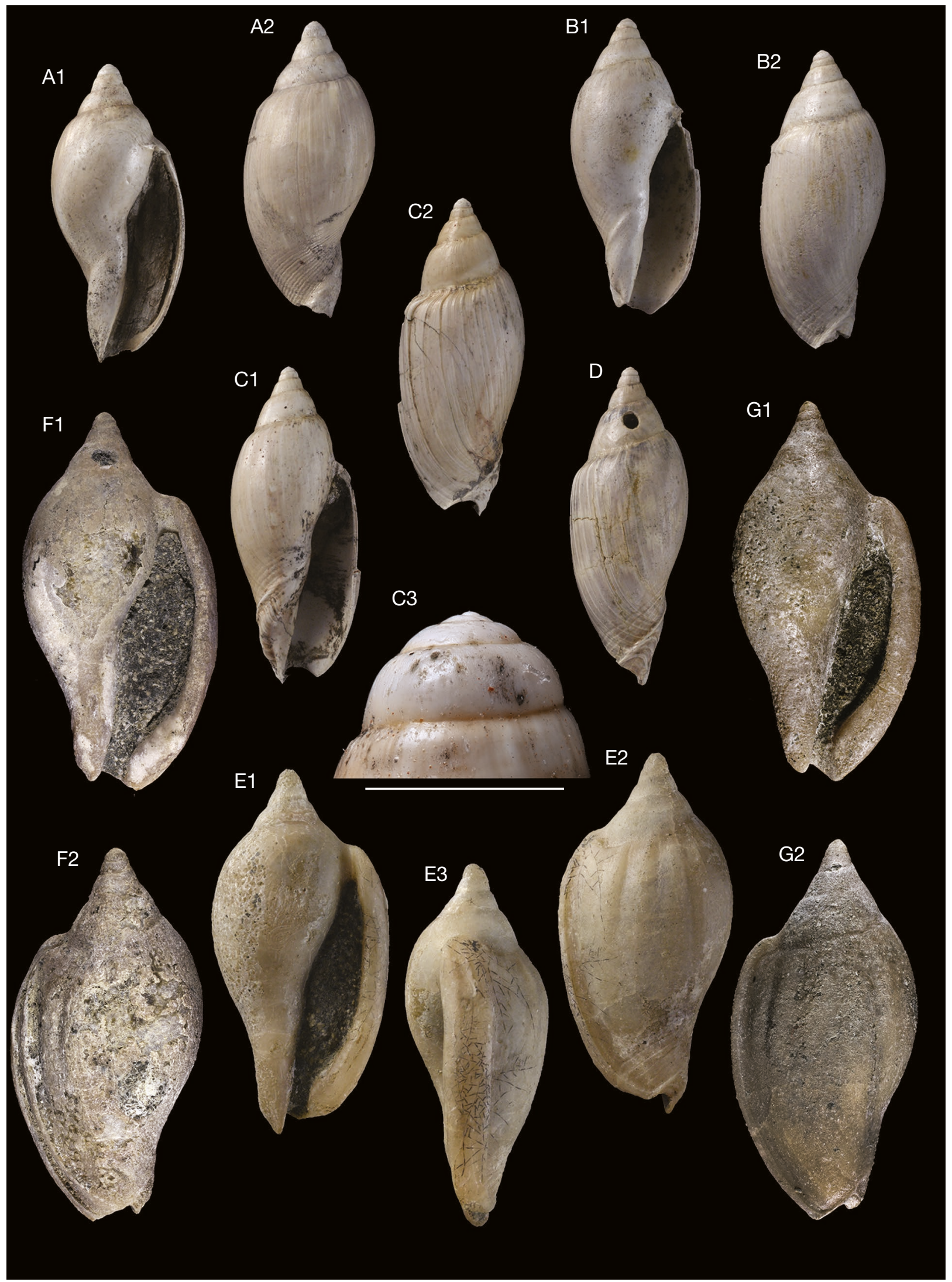

FIG. 10. - A-D, Cryptochorda (s.str.) pyrenaicensis Cossmann, 1923. Cuisien (Yprésien) de la Tuilerie, Gan (Pyrénées-Atlantiques). A, MNHN.F.J10882 (coll. O'Gorman), H. : 24,9 mm; B, MNHN.F.A71481 (coll. O'Gorman), H. : 25,7 mm; C, MNHN.F.A57359 (coll. Pacaud), H. : 34,2 mm - Protoconque; D, MNHN.F.J14638 (coll. Cossmann), H. : 37,2 mm. E-G, Cryptochorda (s.str.) teae n. sp. Cuisien (Yprésien) de Cava Rossi, Monte di Malo (Vicenza, Italie). E, Holotype MGP-PD 31931 (coll. Quaggiotto), H. : 40,2 mm; F, Paratypes MCZ 5761 (coll. Checchi), H. : 37,6 mm; G, MCZ 5762 (coll. Checchi), H. : 38,4 mm. Échelle: C3, 2 mm. 
Cryptochorda (s.str.) pyrenaicensis Cossmann, 1923 (Fig. 10A-D;Fig. 12A-D)

Cryptochorda pyrenaicensis Cossmann, 1923: 125-126, pl. 7, fig. 32, 39 et pl. 8, fig. 18.

Cryptochorda pyrenaicensis — Strausz 1966: 63, 135.

Cryptochorda (s.str.) stromboides pyrenaicensis — Merle 1986: 37.

MAtÉRIEL TYPe. - Syntypes, 2 ex., UBT 29-2-125 (coll. O’Gorman).

LOCALITÉ TYPE. — La Tuilerie, Gan (Pyrénées-Atlantiques), Cuisien (Yprésien, Éocène inférieur).

Dimensions. - Exemplaires figurés par Cossmann (1923). Pl. 7 , fig. 39 et pl. 8 fig. 18, hauteur: $29,0 \mathrm{~mm}$; diamètre: 13,0 mm (pl. 7, fig. 32), hauteur: 35,0 mm; diamètre: 13,0 mm.

Autre matériel eXaminé. - Voir Annexe 3.

\section{DESCRIPTION}

La coquille est petite, peu épaisse, étroite, ovale-oblongue, cylindrique, ayant un angle apical de $60^{\circ}$. La spire est courte, étroite, conique et présente une protoconque de 3 tours $1 / 2$ et une téléoconque de 4 tours peu convexes, hauts, lisses, relativement plans, pourvus d'une rampe suturale étroite, déclive et séparés par une suture superficielle presque entièrement recouverte par le vernis. La protoconque est grosse, légèrement globuleuse, à petit nucléus et aux tours convexes et lisses. La transition protoconque/téléoconque est indistincte. Le dernier tour est très grand et occupe $85 \%$ de la hauteur totale; il se termine par un cou relativement court et muni d'une fasciole siphonale, large, circonscrite par une forte crête postérieure et par un bourrelet antérieur à peine marqué. Le dernier tour est ovale, cylindrique, à peine convexe dans sa partie adapicale, atténué au niveau du cou et orné dans cette région de 5 stries décurrentes très serrées. L'ensemble de la téléoconque est lisse, porcelané et dépourvu de sculpture, on observe seulement de fortes stries d'accroissement, surtout visibles sur les premiers tours, près de la suture. Le dernier tour est également marqué, près du labre, par de nombreux et faibles arrêts de croissance irréguliers. L'ouverture est longue et étroite, rétrécie en arrière et à peine échancrée, présentant une faible gouttière, entaillant l'angle adapical. Le canal siphonal est court et étroit. L'encoche siphonale est étroite et profonde. La columelle est à peine convexe dans la zone pariétale, à peine excavée dans sa partie médiane et légèrement infléchie et rectiligne vers le cou. Elle est lisse et ne montre aucune trace de plis. Le bord de l'ouverture présente une callosité columellaire, d'une couleur blanchâtre, peu étalée latéralement, envahissant la base du pénultième tour et, se pliant alors brusquement en arrière, se dirige parallèlement à la suture et produit une bande spirale étroite sur la partie abapicale des tours. Cette callosité columellaire longe également la columelle jusqu’au canal siphonal et la fasciole. Ce callus, bien appliqué, est peu épais et recouvre à peine les stries décurrentes et les bourrelets postérieur et antérieur de la fasciole siphonale. Le labre est fin et de direction orthocline. Il est fortement sinueux en son extrémité, au niveau de la gouttière entaillant l'angle adapical, il se termine près de l'échancrure siphonale, descendant moins bas que la pointe aiguë de la columelle. L'intérieur de l'ouverture et le bord interne du labre sont lisses et vernissés. Le motif coloré résiduel révélé sous éclairage UV est constitué de quatre alignements spiraux de taches claires et fluorescentes sur un fond sombre. Ces taches sont alignées axialement, petites, étroites et allongées axialement. Ces alignements sont régulièrement espacés: un premier est situé près de la suture; deux autres sont situés sur la partie médiane du dernier tour et enfin la dernière se trouve sur la partie abapicale, sur le cou, au niveau des stries décurrentes. Lintérieur de l'ouverture est très clair et lumineux.

\section{DisCUSSION}

Cette rare espèce se distingue radicalement de Cryptochorda (s.str.) stromboides (Hermann, 1781) par des dimensions plus modestes, par son galbe cylindrique, plus étroit, par sa spire, plus haute, plus étroite, par ses tours moins nombreux, par sa protoconque plus grosse, globuleuse, aux tours plus convexes, par sa rampe suturale étroite et par ses stries décurrentes peu nombreuses. Par ailleurs, son ouverture est longue et étroite, la columelle est à peine convexe dans la zone pariétale, la callosité columellaire est peu étalée latéralement, elle est peu épaisse et recouvre à peine les stries décurrentes et les bourrelets postérieur et antérieur d'une fasciole siphonale, par ailleurs plus large. Enfin, au lieu d'une teinte brune uniforme plus ou moins intense, la coquille montre sous éclairage UV, un motif résiduel de couleur constitué de quatre alignements spiraux de taches claires et fluorescentes sur un fond sombre (Fig. 12A-D) et non «des flammules brunes, obliquement disséminées sur la région antérieure et vernissée de l'avant dernier tour» comme Cossmann (1923: 126) a cru l'observer.

Cryptochorda (s.str.) neptis n. sp. (Figs 11A-J; 12E-J)

urn:Isid:zoobank.org:act:A72BB0F0-1110-4FCE-A5DC-DE4EA4C3B162

Buccinum stromboides variété b - Deshayes 1835: 648.

Buccinum stromboides - Melleville 1837: 248; 1843: 17, 23; 1857: 97. — Bronn 1838: 1096, n 1 (partim); 1856: 532 (partim). d'Archiac 1843: 273. — Graves 1847: 629, n 713 (partim). Watelet 1855: 58; 1870: 14. - Bonissent 1868: 24. - Meunier 1875: 151. — Lhomme 1904: 105 (non Hermann, 1781).

Buccinum stromboides 1e variété - Deshayes 1865: 495.

Cryptochorda stromboides - Cossmann 1889: 192-193 (partim); 1899: 78 (partim). — Chédeville 1904: 419 (partim). — Furon \& Soyer 1947: 70. - Feugueur 1963: 36, 42, 71. — Fischer 1980: 320 (partim); 2000: 346 (partim). — Glibert 1985: 336 (partim). — Hessel 2016: 85 (non Buccinum stromboides Hermann, 1781).

Cryptochorda aff. stromboides - Van Nieulande 2002: 33.

MATÉRIEL TYPE. - Holotype: MNHN.F.A71442 (coll. Pacaud); paratypes: 4 ex., MNHN.F.A71443, A71444, A71446, A71449 (coll. Pacaud), 1 ex., MNHN.F.A71465 (coll. Pons), 5 ex., MNHN.F.A71445, A71447, A71448, A71450, A71451 (coll. Romanek).

LOCALITÉ TYPE. - Saint-Gobain (Aisne, France), Cuisien (Yprésien, Éocène inférieur).

Etymologie. - Du latin «nièce». Allusion à la similarité de la coquille avec celle de $C$. stromboides. 


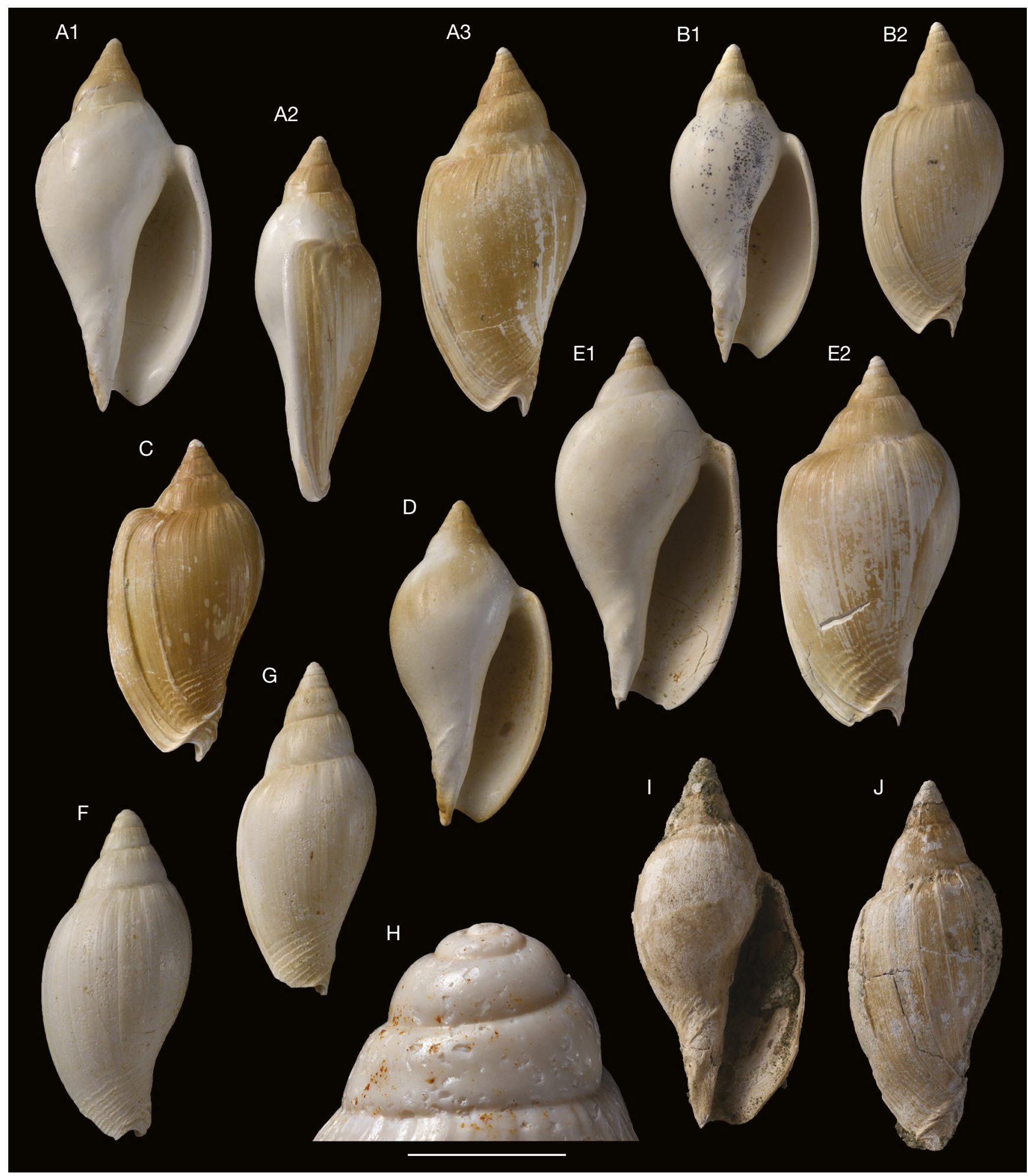

FiG. 11. - A-J, Cryptochorda (s.str.) neptis n. sp. A-H, Cuisien (Yprésien) de Saint-Gobain (Aisne); I-J, Panisélien (Yprésien) d’Egem (Belgique). A, Holotype MNHN.F.A71442 (coll. Pacaud), H. : 37,1 mm; B, Paratype MNHN.F.A71443 (coll. Pacaud), H. : 37,5 mm; C, Paratype MNHN.F.A71444 (coll. Pacaud), H. : 34,0 mm; D, Paratype MNHN.F.A71445 (coll. Romanek), H. : 30,1 mm; E, Paratype MNHN.F.A71446 (coll. Pacaud), H. : 43,3 mm; F, spécimen juvénile, Paratype MNHN.F.A71447 (coll. Romanek), H. : 18,7 mm; G, spécimen juvénile, Paratype MNHN.F.A71448 (coll. Romanek), H. : 29,2 mm; H, Paratype MNHN.F.A71449 (coll. Pacaud), protoconque; I, MNHN.F.A71462 (coll. Merle), H. : 33,6 mm; J, MNHN.F.A71463 (coll. Merle), H. : 35,4 mm. Échelle: H, 2 mm.

Dimensions. - (Holotype) hauteur: 37,1 mm; diamètre: 17,3 mm.

Autre matériel eXAminé. - Voir Annexe 4.
DESCRIPTION

La coquille est petite, peu épaisse, étroite, ovale-oblongue, ayant un angle apical de $55^{\circ}$. La spire est courte, étroite, 
conique et présente une protoconque de 3 tours $1 / 2$ et une téléoconque de 5 tours peu convexes, relativement plans, hauts, lisses et séparés par une suture superficielle presque entièrement recouverte par le vernis. La protoconque est petite, légèrement globuleuse, à petit nucléus et aux tours convexes et lisses. La transition protoconque/téléoconque est indistincte, Le dernier tour est très grand et occupe $81 \%$ de la hauteur totale; il se termine par un cou relativement court et muni d'une fasciole siphonale, étroite, circonscrite une forte crête postérieure et par un bourrelet antérieur à peine marqué. Le dernier tour est ovale, étroit, à peine convexe dans sa partie adapicale, atténué au niveau du cou et orné dans cette région d'environ 8 stries décurrentes très serrées. L’ensemble de la téléoconque est lisse, porcelané et dépourvu de sculpture, on observe seulement de fortes stries d'accroissement, surtout visibles sur les premiers tours, près de la suture. Le dernier tour est également marqué, près du labre, par de faibles arrêts de croissance irréguliers. L'ouverture est longue et étroite, rétrécie en arrière et à peine échancrée, présentant une faible gouttière, entaillant l'angle adapical. Le canal siphonal est court et étroit. L'encoche siphonale est étroite et profonde. La columelle est légèrement convexe dans la zone pariétale, à peine excavée dans sa partie médiane et légèrement infléchie et rectiligne vers le cou. Elle est lisse et ne montre aucune trace de plis. Le bord de l'ouverture présente une callosité columellaire, d'une couleur blanchâtre, très largement étalée latéralement, envahissant la base du pénultième tour et, se pliant alors brusquement en arrière, se dirige parallèlement à la suture et produit une bande spirale étroite sur la partie abapicale des tours. Cette callosité columellaire longe également la columelle jusqu'au canal siphonal et la fasciole. Ce callus, bien appliqué, est peu épais au niveau de l'ouverture et recouvre à peine les stries décurrentes et les bourrelets postérieur et antérieur de la fasciole siphonale. Le labre, de direction orthocline, est bordé par une très forte varice. Il présente un contour légèrement convexe en vue aperturale sur les $3 / 4$ de sa longueur, puis montre une légère inflexion dans la zone abapicale. Il est fortement sinueux en son extrémité, au niveau de la gouttière entaillant l'angle adapical, il se termine près de l'échancrure siphonale, descendant plus bas que la pointe aiguë de la columelle. L'intérieur de l'ouverture et le bord interne du labre sont lisses et vernissés. La coquille conserve très rarement sa couleur d'origine, une teinte uniforme d'un brun très clair. Par ailleurs, elle présente également un motif résiduel de couleur, observé sous éclairage UV. Il est constitué de quatre alignements spiraux de taches claires et fluorescentes sur un fond sombre. Ces taches sont allongées axialement, subquadrangulaires et sont alignées axialement. Ces alignements sont régulièrement espacés : un premier est situé près de suture; deux autres sont situés sur la partie médiane du dernier tour et enfin la dernière se trouve sur la partie abapicale, sur le cou, au niveau des stries décurrentes. L'intérieur de l'ouverture est très clair et lumineux.

\section{DisCUSSION}

Deshayes (1835: 647) en a donné les caractères : «La seconde variété vient des sables inférieurs du Soissonnais: elle est toujours plus petite et plus étroite» et en a fait une variété (non dénommée). Cossmann (1923: 125) notait aussi que la «mutation cuisienne» de Cryptochorda (s.str.) stromboides « est déjà plus étroite que le type lutétien, et son ouverture moins ample se rétrécit proportionnellement moins aux abords de l'échancrure». En fait, bien plus qu'une "mutation ancestrale», comme Cossmann l'écrit également, il s'agit d'une espèce très différente par bien des aspects. Elle s'en distingue par sa taille plus petite, par son galbe plus étroit, par sa spire plus haute et plus étroite. Son ouverture est longue et étroite, la columelle est à peine convexe dans la zone pariétale, la callosité columellaire est peu épaisse et recouvre à peine les stries décurrentes et les bourrelets de la fasciole siphonale. Par ailleurs, le labre, de direction orthocline, est bordé par une très forte varice; épaississement totalement absent chez $C$. (s.str.) stromboides. Enfin, au lieu d'avoir seulement une teinte brune plus ou moins intense, la coquille montre non seulement une teinte uniforme d'un brun très clair mais aussi, sous éclairage UV, un motif résiduel de couleur constitué de quatre alignements spiraux de taches claires et fluorescentes sur un fond sombre (Fig. 12E-J). Ce motif diffère nettement de celui de Cryptochorda (s.str.) altavesna n. sp. (Fig. 9A-O) par ses taches allongées axialement, subquadrangulaires et étroites. Nous avons également observé (Fig. 11I-J) cette espèce nouvelle dans les sables paniséliens (Yprésien, Éocène inférieur) d'Egem (Belgique) où l'espèce $C$. (s.str.) stromboides avait déjà été signalée, avec doute, par Van Nieulande (2002: 33).

Cryptochorda (s.str.) cosediensis n. sp. (Figs 12K-M; 13A-G)

urn:Isid:zoobank.org:act:FE578A73-A342-4D5E-A806-CC1BBE2944A5

Buccinum stromboides - de Gerville 1817: 199 (non Hermann, 1781).

Buccinus [sic] tromboides - Bonissent 1868: 18 (non Hermann, 1781) (orthographe subséquente incorrecte).

Buccinum tromboides - Bonissent 1870: 333 (non Hermann, 1781) (orthographe subséquente incorrecte).

Cryptochorda stromboides-Cossmann \& Pissarro 1901:33-34, pl. 10, fig. 5. - Stenzel \& Turner 1940: 795, 802, pl. 43, fig. 1. — Glibert 1960: 48 (partim); 1985: 336 (partim) (non Buccinum stromboides Hermann, 1781)

MATÉRIEL TYPE. - Holotype: MNHN.F.B65189 (coll. de Morgan); paratypes: 4 ex., MNHN.F.A71456, A71457, A71461 et A71483 (coll. de Morgan), 1 ex., MNHN.F.A05108 (coll. Le Marchand), 1 ex., MNHN.F.J14642 (coll. Cossmann).

LOCALITÉ TYPE. — Lutétien (Éocène moyen) de Fresville (Manche, France).

ÉTYMologie. - De Cosedia, cité des Venelles, tribu gauloise du groupe des Armoricains qui occupaient le Cotentin et le nord du département de la Manche. La cité prends le nom de Constantia en 298 sous l'empereur romain Constance Chlore (Gaius Flavius 


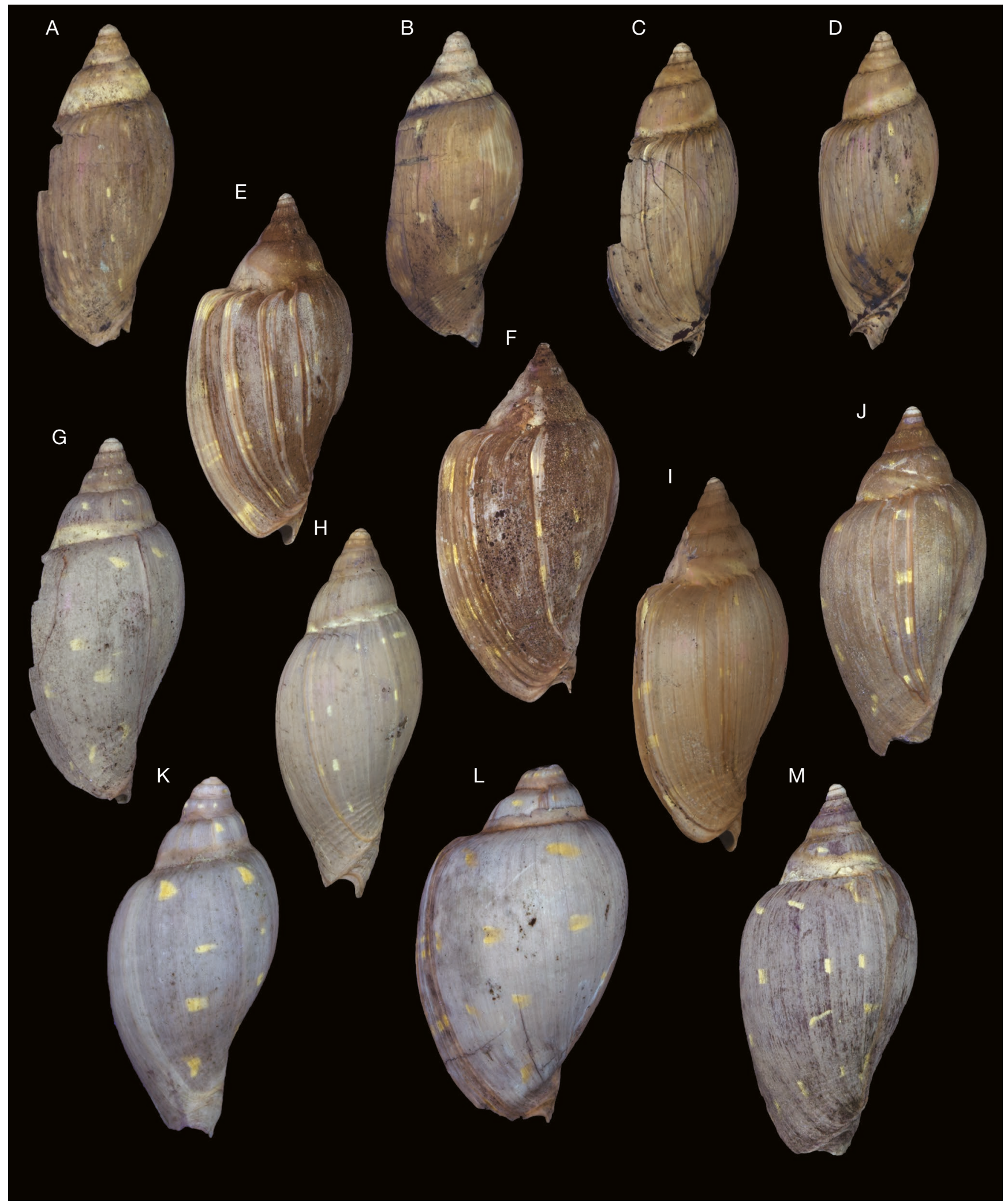

FIG. 12. - Motifs colorés résiduels révélés sous lumière UV sur des spécimens juvéniles et adultes. A-D, Cryptochorda (s.str.) pyrenaicensis Cossmann, 1923. Cuisien (Yprésien) de la Tuilerie, Gan (Pyrénées-Atlantiques). E-J. Cryptochorda (s.str.) neptis n. sp. E-G, Cuisien (Yprésien) de Mons-en-Laonnois (Aisne); H-J, Cuisien (Yprésien) de Saint-Gobain (Aisne). K-M, Cryptochorda (s.str.) cosediensis n. sp. K, M, Lutétien de Fresville (Manche) ; L, Lutétien de HauttevilleBocage (Manche). A, MNHN.F.A71481 (coll. O'Gorman), H. : 24,9 mm; B, MNHN.F.J10882 (coll. O'Gorman), H. : 25,7 mm; C, MNHN.F.A57359 (coll. Pacaud), H. : 34,2 mm; D, MNHN.F.J14638 (coll. Cossmann), H. : 37,2 mm; E, MNHN.F.A71452 (coll. Lhomme), H. : 37,3 mm; F, MNHN.F.A71453 (coll. Lhomme), H. : 40,7 mm; G, MNHN.F.A71454 (coll. Lhomme), H. : 26,4 mm; H, Paratype MNHN.F.A71450 (coll. Romanek), H. : 29,0 mm; I, Paratype MNHN.F.A71451 (coll. Romanek), H. : 34,9 mm; J, MNHN.F.A71455 (coll. Pacaud), H. : 30,0 mm; K, MNHN.F.A71458, H. : 19,1 mm; L, MNHN.F.A71460, H. : 42,5 mm; M, Paratype MNHN.F.A71461 (coll. de Morgan), H. : 32,9 mm. 
Valerius Constantius). La région connue sous le nom de Pagus Constantinus deviendra le Cotentin.

DimENSIONS. - (Holotype) hauteur: 49,8 mm; diamètre: 25,0 mm.

Autre matériel examiné. - Voir Annexe 5.

\section{DESCRIPTION}

La coquille est grande, très épaisse, étroite, ovale-oblongue, ayant un angle apical de $65^{\circ}$. La spire est courte, étroite, conique et présente une protoconque de 3 tours $1 / 2$ et une téléoconque de 4 tours peu convexes, lisses, parcouru de lignes d'accroissements excessivement fines, relativement plans et séparés par une suture superficielle presque entièrement recouverte par le vernis. La protoconque est petite, légèrement globuleuse, à petit nucléus et aux tours convexes et lisses. La transition protoconque/téléoconque est indistincte, Le dernier tour est très grand et occupe $85 \%$ de la hauteur totale; il se termine par un cou relativement court et muni d'une fasciole siphonale, large, circonscrite une forte crête postérieure et par un bourrelet antérieur à peine marqué. Le dernier tour est ovale, à peine convexe dans sa partie adapicale, atténué au niveau du cou et orné dans cette région de 2 à 3 stries décurrentes très serrées et à peine visibles. L'ensemble de la téléoconque est lisse, porcelané et dépourvu de sculpture, on note seulement de fines stries d'accroissement prosocyrtes, surtout visibles sur les premiers tours, près de la suture. L'ouverture est longue et étroite, rétrécie en arrière et à peine échancrée, présentant une faible gouttière, entaillant l'angle adapical. Le canal siphonal est court et étroit. L'encoche siphonale est large et profonde. La columelle est à peine convexe dans la zone pariétale, fortement excavée dans sa partie médiane et légèrement infléchie et rectiligne vers le cou où elle est comprimée dorso-ventralement. Elle est lisse et ne montre aucune trace de plis. Le bord de l'ouverture présente une callosité columellaire, d'une couleur blanchâtre, très largement étalée latéralement, envahissant la base du pénultième tour et, se pliant alors brusquement en arrière, se dirige parallèlement à la suture et produit une bande spirale étroite sur la partie abapicale des tours. Cette callosité columellaire longe également la columelle jusqu'au canal siphonal et la fasciole. Ce callus, bien appliqué, est peu épais et recouvre les stries décurrentes et les bourrelets postérieur et antérieur de la fasciole siphonale. La face ventrale présente, dans sa partie adapicale et un peu en retrait de la partie postérieure de la columelle, un bourrelet calleux, épais, oblique, incliné vers l'ouverture. Le labre, de direction orthocline, est bordé par une très forte et large varice. Il présente un contour légèrement convexe en vue aperturale sur les $3 / 4$ de sa longueur, puis montre une légère inflexion dans la zone abapicale. Il est fortement sinueux en son extrémité, au niveau de la gouttière entaillant l'angle adapical, il se termine près de l'échancrure siphonale, descendant moins bas que la pointe aiguë de la columelle. L'intérieur de l'ouverture et le bord interne du labre sont lisses et vernissés. La coquille conserve très rarement sa couleur d'origine, une teinte uniforme d'un brun très clair. Par ailleurs, elle présente également un motif résiduel de couleur, observé sous éclairage UV. Il est constitué de quatre alignements spiraux de taches claires et fluorescentes sur un fond sombre. Ces taches sont allongées axialement, subquadrangulaires et elles sont alignées axialement. Ces alignements sont régulièrement espacés: un premier est situé près de suture; deux autres sont situés sur la partie médiane du dernier tour et enfin la dernière se trouve sur la partie abapicale, sur le cou. Les taches de l'alignement adapical, sous la suture, sont étroites et plus ou moins déformées. Lintérieur de l'ouverture est très clair et lumineux.

\section{DisCUSSION}

Par sa forme générale cette espèce rappelle surtout l'espèce du Cuisien (Yprésien, Éocène inférieur) du bassin de Paris que nous venons de décrire. Sur les spécimens adultes et parfaitement conservés de Cryptochorda (s.str.) neptis n. sp., le labre est bordé par une très forte et large varice; ce caractère est partagé avec C. (s.str.) cosediensis n. sp. Par ailleurs, les coquilles présentent un galbe analogue, étroit, ovale-oblongue, la spire est cependant moins étroite et moins longue sur l'espèce du Cotentin. Les tours sont peu convexes, lisses et parcouru de lignes d'accroissements excessivement fines. Le motif résiduel de couleur est toutefois consititué chez $C$. (s.str.) cosediensis $\mathrm{n}$. sp. de taches allongées subquadrangulaires plus larges (Fig. 12K$\mathrm{M})$. Elle diffère de C. (s.str.) stromboides par une coquille au test bien plus épais, par un galbe étroit, ovale-oblongue, par une ouverture au labre bordé par une très forte et large varice et par la présence de motif résiduel de couleur.

\section{Cryptochorda (s.str.) teae n. sp.} (Fig. 10E-G)

urn:Isid:zoobank.org:act:B0954CAC-79FD-4524-AF5E-6201D685D560

MatéRIel tYPe. - Holotype: MGP-PD 31931 (coll. Quaggiotto); paratypes: 3 ex., MCZ 5761, MCZ 5762 et MCZ 5763 (coll. Checchi).

LOCALITÉ TYPE. - Yprésien supérieur (Éocène inférieur) de Cava Rossi, Monte di Malo (Vicenza, Italie).

ÉTYMOLOGIE. - Espèce dédiée à Tea Quaggiotto qui a découvert l'holotype et, à travers sa fille, à Ermanno Quaggiotto, pour son travail sur la faune éocène d'Italie.

Dimensions. - (Holotype) Hauteur: 40,2 mm. Diamètre: 19,7 mm.

\section{DESCRIPTION}

La coquille est de taille moyenne, au test très épais et pesant, ovale-oblongue et un peu ventrue, ayant un angle apical de $65^{\circ}$. La spire est courte, conique et présente une protoconque d'au moins 3 tours et une téléoconque de 4 tours légèrement convexes, lisses et séparés par une suture superficielle presque entièrement recouverte par le vernis. La protoconque est large, globuleuse, à petit nucléus et aux tours convexes et lisses. La transition protoconque/ téléoconque est indistincte. Le dernier tour est très grand et occupe $86 \%$ de la hauteur totale; il se termine par un cou relativement court et muni d'une fasciole siphonale, étroite, circonscrite par deux fortes crêtes. Le dernier tour 


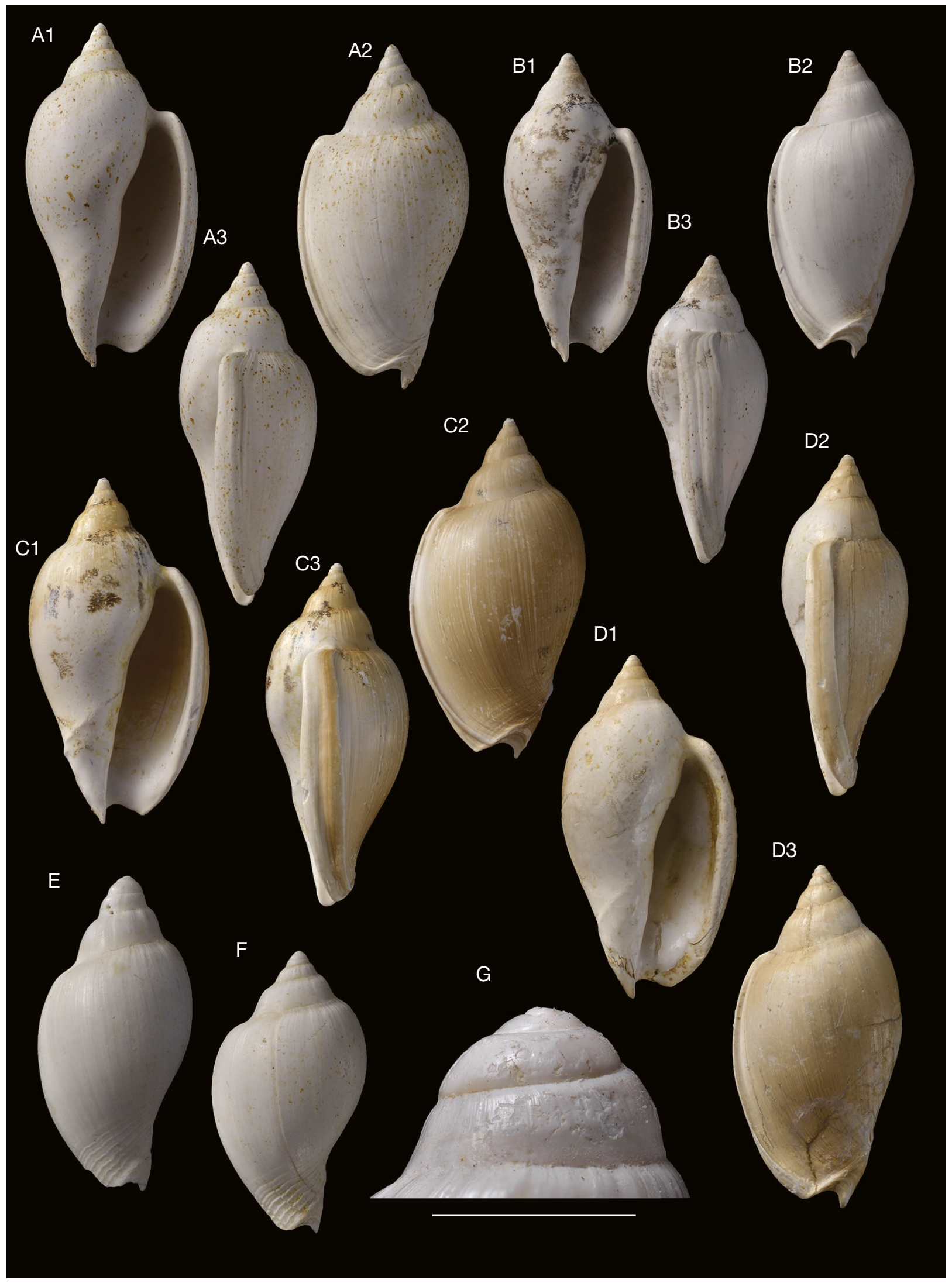

FIG. 13. - A-G, Cryptochorda (s.str.) cosediensis n. sp. A-D, Lutétien de Fresville (Manche); E-G, Lutétien de Hautteville-Bocage (Manche). A, Holotype MNHN.F.B65189 (coll. de Morgan), H. : 49,8 mm; B, Paratype MNHN.F.A71456 (coll. de Morgan), H. : 35,5 mm; C, Paratype MNHN.F.A71457 (coll. de Morgan), H. : 44,3 mm; D, Paratype MNHN.F.A05108 (coll. Le Marchand), H. : 39,2 mm; E, spécimen juvénile, MNHN.F.A71458, H. : 19,1 mm; F, spécimen juvénile, MNHN.F.A71459, H. : 30,7 mm; G, MNHN.F.A71458, protoconque. Échelle: G, 2 mm. 


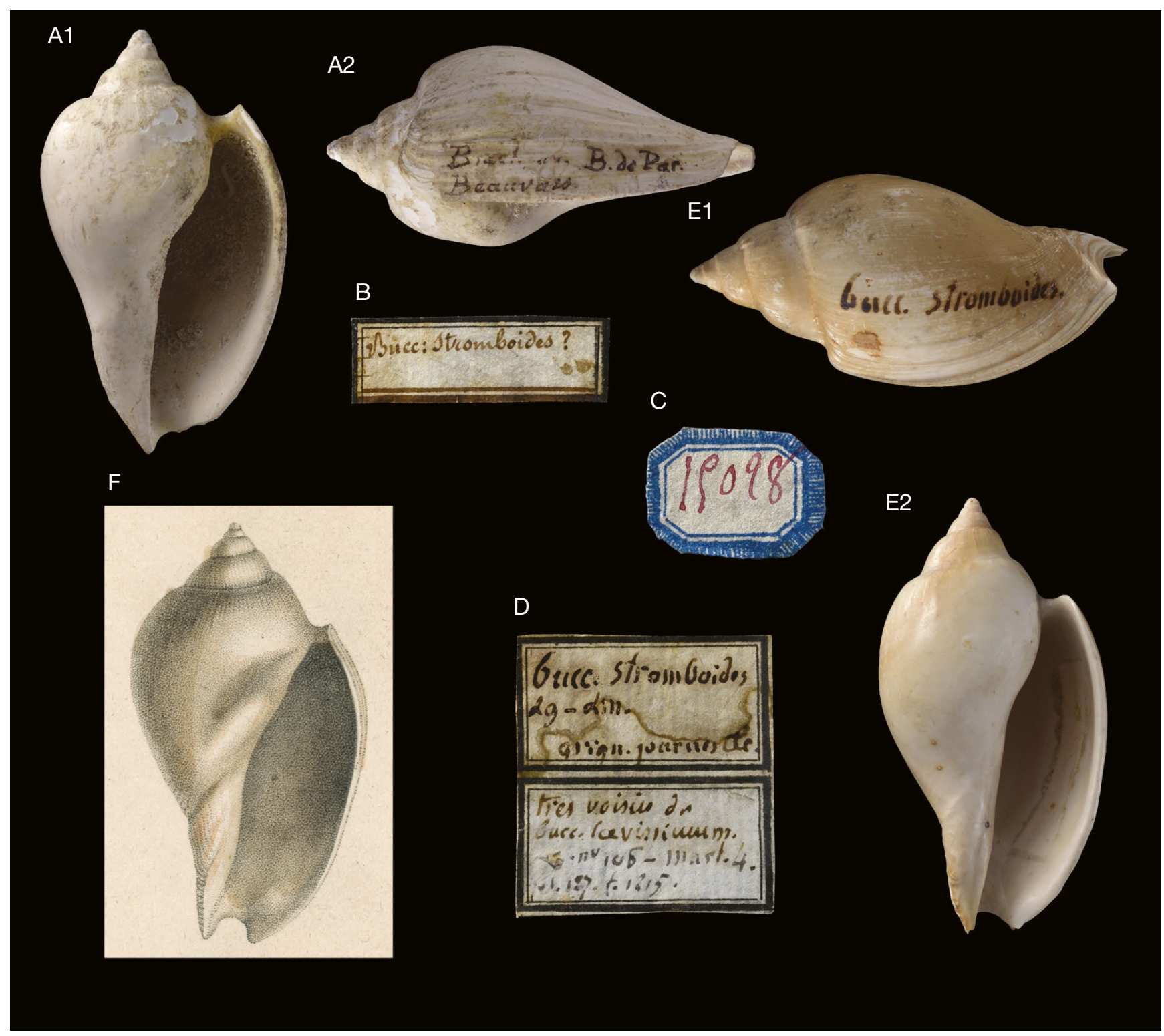

FIG. 14. - A, Cryptochorda (s.str.) altavesna n. sp. Lutétien de Parnes (Oise). MNHN.F.A57647 (coll. Brongniart), H. : 45,5 mm; B-D, étiquettes originales de la collection Brongniart; E, Cryptochorda (s.str.) stromboides (Hermann, 1781), Lutétien de Thiverval-Grignon (Yvelines). MNHN.F.A57591 (coll. Brongniart), H. : 49,4 mm; F, Cryptochorda (s.str.) altavesna n. sp. Figure originale de Buccinum stromboides " variété a " Deshayes, 1835. D'après Deshayes (1853).

est ventru dans sa partie adapicale, atténué au niveau du cou et orné dans cette région de 5 stries décurrentes très serrées. L'ensemble de la téléoconque est lisse et on observe seulement, sur la face dorsale du dernier tour, une ornementation axiale constituée de larges côtes qui disparaissent sur plus des deux tiers abapicaux. L'ouverture est oblongue, large dans sa partie médiane, étroite en avant et profondément échancrée, présentant un étroit canal, entaillant l'angle adapical. Le canal siphonal est court et étroit. L'encoche siphonale est étroite et profonde. La columelle est légèrement convexe dans la zone pariétale, à peine excavée dans sa partie médiane et légèrement infléchie et rectiligne vers le cou. Elle est lisse et ne montre aucune trace de plis. Le bord de l'ouverture présente une callosité columellaire, peu étalée latéralement, envahissant la base du pénultième tour et, se pliant alors brusquement en arrière, se dirige parallèlement à la suture et produit une bande spirale étroite sur la partie abapicale des tours. Cette callosité columellaire longe également la columelle jusqu'au canal siphonal et la fasciole. Ce callus, bien appliqué, est peu épais et recouvre les stries décurrentes et les crêtes postérieure et antérieure de la fasciole siphonale. Le labre, de direction orthocline, est bordé par une très forte et large varice. Il présente un contour régulièrement convexe en vue aperturale sur les $3 / 4$ de sa longueur, puis montre une légère inflexion dans la zone abapicale. Il est fortement sinueux en son extrémité, au niveau du canal entaillant l'angle adapical, il se termine près de l'échancrure siphonale, descendant moins bas que la pointe aiguë de la columelle. Le bord interne du labre porte plusieurs forts denticules. 


\section{DisCUSSION}

Par sa forme générale et par l'épaississement variqueux du labre, Cryptochorda (s.str.) teae n. sp. rappelle surtout l'espèce du Cuisien (Yprésien, Éocène inférieur) du bassin de Paris et celle du Lutétien (Éocène moyen) du Cotentin que nous venons de décrire. Elle en diffère cependant radicalement par la présence d'une ornementation axiale, par un étroit canal entaillant l'angle adapical et par un labre portant sur son bord interne des denticules. Elle diffère également de l'espèce type Cryptochorda (s.str.) stromboides (Hermann, 1781) par ces mêmes caractères ainsi que par une ouverture plus étroite, plus oblongue, au labre bordé par une très forte et large varice.

\section{CONCLUSION}

L'observation sous lumière UV des motifs colorés résiduels sur les coquilles de très nombreux exemplaires fournit un nouveau corpus de caractères permettant d'enrichir et d'affiner la description des espèces du Cénozoïque. La réévaluation de la paléobiodiversité des Cryptochorda (s.str.) de l'Éocène $\mathrm{du}$ bassin de Paris, de Loire-Atlantique, du Cotentin et du Béarn, permet ainsi l'identification de cinq espèces, et non plus d'une seule qui aurait vécu de l'Yprésien jusqu'au Bartonien. Pour caractériser les espèces de ce genre de mollusques, les paléontologues ne se sont appuyés que sur la morphologie de la coquille, ne percevant pas malgré tout les differents caractères de ces coquilles. Les informations apportées ici dans l'observation de coquilles fossiles du genre Cryptochoda (s.str.), même si les motifs résiduels appartiennent à un même type de motif (quatre alignements spiraux de taches claires et fluorescentes sur un fond sombre) permettent ainsi, en l'absence d'intermédiaires entre les motifs, de confirmer la séparation des taxa Cryptochorda (s.str.) altavesna n. sp., C. (s. str.) neptis $\mathrm{n}$. sp. et $C$. (s.str.) cosediensis $\mathrm{n}$. sp., que les caractères conchyliologiques nous permettaient déjà d'établir. L'espèce type C. (s.str.) stromboides ne présente quant à elle qu'une teinte uniforme allant d'un brun clair, ambrée, à une teinte brun-rougeâtre plus ou moins intense mais surtout aucun motif de couleur comme ses congénaires; l'observation sous lumière UV en montre une totale absence (Fig. 9P).

La répartition géographique de ces espèces est également à mettre en avant. En effet, C. (s.str.) stromboides est largement réparti dans les formations calcaires sableuses a Milioles des gisements du Lutétien moyen dans tout le bassin de Paris, jusqu'au fond du golfe de la mer lutétienne à l'est, où l'espèce est caractéristique de la région champenoise. Le taxon Cryptochorda (s.str.) altavesna n. sp. est, quant à lui, nettement plus limité dans sa répartition géographique. En effet, on rencontre cette espèce essentiellement au nord-ouest du Bassin parisien dans le Vexin (synclinal du Thérain), jusqu'au sud-ouest du Bassin parisien dans le Hurepoix (synclinal de l'Eure), ou elle est syntopique de C. (s.str.) stromboides dans les gisements de Authevernes, de Parnes et de Fontenay-en-Vexin. Les taxons C. (s.str.) neptis n. sp. et C. (s.str.) cosediensis n. sp. montrent une répartition géographique plus limitée encore. Sur le plan spatial, C. (s.str.) stromboides est présente dans la quasi-totalité des gisements, se rencontrant préférentiellement à l'est du bassin (gisements situés dans la Marne) et au centre du bassin (gisements situés dans les Yvelines). Cryptochorda (s.str.) altavesna n. sp. montre quant à elle une zone préférentielle, le Vexin.

\section{Remerciements}

Nous remercions Philippe Loubry et Lilan Cazes (MNHN/ CNRS) pour les photographies et l'infographie des planches, Marie-Dominique Wandhammer (conservatrice du Musée zoologique de Strasbourg) et Kévin Janneau (chargé des collections de paléontologie de l'université de Strasbourg), ainsi que Dorothée Rusque (Strasbourg), pour leur aide dans la recherche du matériel type et des notes scientifiques de Jean Hermann, Peter Massicard et Marie-Béatrice Forel (MNHN) pour le résumé en anglais et pour la réalisation du tableau sur l'étude biométrique, Ermanno Quaggiotto, Andrea Checchi, Daniel Ledon, Jacques Mouchart, Christian Romanek, Pascal Boucher, Jean-Claude Six, Didier Vyncke, Jean-Marie Canevet, et Luc Danvin pour les informations sur le matériel de leur collection. Nous remercions également Patrice Bail et Loïc Limpalaër pour leurs judiceuses critiques sur le manuscrit. Le MNHN donne accès aux collections dans le cadre de l'Infrastructure de Recherche nationale RECOLNAT.

\section{RÉFÉRENCES}

AleXeEv A. K. 1963. - Paleogenovaja Fauna Molljuskov severogo Priaraljja [Paleogene Molluscan Fauna of the Northern Urals Region]. Akademija Nauk Armanskoj SSR, Yerevan, 176 p., 24 pls.

Ansted D. T. 1844. - Geology, Introductory, Descriptive and Practical. Volume 2. J. van Voorst, London, 572 p.

ANTON H. E. 1838. - Verseichniss der Conchylien welche sich in der Sammlung von Hermann Eduard Anton befinden. Herausgegeben von dem Besitzer. Anton, Halle, xvi +110 p.

ArChiac A. Desmier DE SAINT-SimOn D' 1843. - Description géologique du département de l'Aisne. Mémoire de la Société géologique de France 5 (2): 129-425, pl. 21-31. https://www. biodiversitylibrary.org/page/42338291

DE Beaurepaire F. 1981. - Les noms des communes et anciennes paroisses de l'Eure. A. \& J. Picard, Paris, 221 p.

Bernard F. 1895. - Éléments de Paléontologie. Baillière, Paris, 1168 p., 606 figs.

Bone A. E. \& BONE D. A. 1985. - Fossils from Bracklesham to Selsey. Chichester District Museum, Chicherster, $32 \mathrm{p}$.

BONISSENT M. 1868. — Essai géologique sur le département de la Manche (suite). 10ème époque, Sol tertiaire, Terrain tertiaire. Mémoires de la Société impériale des Sciences naturelles de Cherbourg 2 ème série, 13 (3): 5-34. https://www.biodiversitylibrary. org/page/ 12207542

BONISSENT M. 1870. - Essai géologique sur le département de la Manche. Ch. Feuarden, Cherbourg, 427 p.

BOYER F. 1976. - Un voyage scientifique après Campo Formio: Trois lettres de Barthélémy Faujas de Saint-Fond à ses collègues du Muséum. Revue d'Histoire des Sciences 29 (4): 325-336.

BréBION P. 1982. - Gastéropodes, in BlondEAU A., BrÉBION P., Buge E., Chevalier J.-P., Damotte R., Lauriat-Rage A., Le Calvez Y., Roman J., Ters M. \& Viaud J.-M. (eds), Le Lutétien supérieur de Pierre-Aiguë près de Saint-Aignan-Grand-Lieu (Loire-Atlantique). Bulletin du Bureau de Recherches géologiques et Minières, 2ème série, 1 (1-2): 129-133, tabl. 6, pl. 2. 
BRIart A. \& CoRnet F.-L. 1871. - Description des fossiles du calcaire grossier de Mons. Première partie: Gastéropodes. Ordre I. - Prosobranches. Section A. - Siphonostomes. Mémoires couronnés et Mémoires des Savants étrangers, Académie royale des Sciences, des Lettres et des Beaux-Arts de Belgique 36: i-viii + 1-76, pl. 1-5.

Briart A. \& Cornet F.-L. 1874. - Aperçu sur la géologie des environs de Mons. Bulletin de la Société géologique de France 3 (2): 534-553.

Briart A. \& Cornet F.-L. 1877. - Description des fossiles du calcaire grossier de Mons. Troisième partie. Supplément aux deux premières parties. Mémoires couronnés et Mémoires des Savants Étrangers, Académie Royale des Sciences, des Lettres et des BeauxArts de Belgique 43 (3): i-viii + 1-73, pl. 13-18.

BRONGNIART A. 1844. - Traité des arts céramiques ou des poteries: considérée dans leur histoire, leur pratique et leur théorie. Volume 2. Béchet jeune, Paris, 706 p. https://gallica.bnf.fr/ ark:/12148/bpt6k67829t

BronN H. G. 1838. - Lethaea Geognostica, oder Abbildungen und Beschreibung der für Gebirgsformationen bezeichnendsten Versteinerungen. Zweiter Band. Schweizerbart, Stuttgart, 1346 p. https://doi.org/10.5962/bhl.title.59080

BronN H. G. 1856. - Lethaea Geognostica oder Abbildungen und Beschreibung der für Gebirgs-formationen bezeichnendsten Versteinerungen. Dritter Band. E. Schweizerbart, Stuttgart, 1130 p.

BUC'HOZ P.-J. 1778. - Première centurie de planches enluminées et non enluminées, représentant au naturel ce qui se trouve de plus intéressant et de plus curieux parmi les animaux, les végétaux et les minéraux, pour servir d'intelligence à l'Histoire générale des trois règnes de la nature, Décade première. Règne animal. Lacombe et l'Auteur, Paris, 100 pls. https://gallica.bnf.fr/ark:/12148/ bpt6k10665984

BUREAU L. 1900. - Notice sur la Géologie de la Loire-Inférieure. Grimaud \& Fils, Nantes, 522 p., 4 pls.

CARPENTER P. P. 1861. — Lectures on Mollusca; or "Shell-fish" on their allies. Annual report of the Board of Regents of the Smithsonian Institution showing the operations, expenditures, and condition of the institution for the year 1860: 151-283. https:// www.biodiversitylibrary.org/page/7920829

Carpita V., Willmann R. \& Willmann S. 2009. — Shells, Muscheln Coquillages. Conchology or the Natural History of Sea, Freshwater, Terrestrial and Fossil Shells 1780. Taschen, Hong Kong, Köln, London, Los Angeles, Madrid, Paris, Tokyo, 216 p., 80 pls.

CATE J. M. 1972. - Notes on the Fauna of the Chipola Formation - X. On the occurrence of the Volutid subgenus Enaeta. Tulane studies in Geology and Paleontology 10 (1): 47-50.

Caze B., Merle D., Pacaud J. M. \& Saint Martin J.-P. 2010. First systematic study using the variability of the residual colour patterns: the case of the Paleogene Seraphsidae (Gastropoda, Stromboidea). Geodiversitas 32 (3): 417-477. https://doi. org/10.5252/g2010n3a4

Caze B., Merle D., Le Meur M., Pacaud J.-M. \& Saint Martin J.-P. 2011a. - Taxonomic implications of the residual colour patterns in Ampullinid gastropod and their contribution to the discrimination from naticid. Acta Paleontologica Polonica 56 (2): 353-371. https://doi.org/10.4202/app.2009.0084

Caze B., Merle D., Saint Martin J.-P. \& Pacaud J.-M. 2011b. Contribution of residual colour patterns to the species characterization of Caenozoic molluscs (Gastropopoda, Bivalvia), in Néraudeau D. (ed.), The species concept in palaeontology: ontogeny, variability, evolution. Comptes Rendus Palevol 10 (2-3): 171-179. https://doi.org/10.1016/j.crpv.2010.10.005

Chavan A. \& Montocchio H. 1968. - Fossiles classiques. Enchaînements et détermination (350 espèces décrites avec illustrations en regard), 4ème édition revue et augmentée. Société d'Édition d'enseignement supérieur, Paris, 238 p., 362 figs.

Chédeville P.-J. 1904. - Liste générale et synonymique des fossiles tertiaires du bassin de Paris avec la nomenclature des localités et gisements où ils ont été trouvés jusqu'en 1900, d'après les ouvrages de Lamarck, d'Orbigny, Deshayes, Cossmann, etc. Bulletin de la Société des Sciences naturelles d'Elbeuf 22: 375-438. Clark B. L. 1946. - Part. 1 - The Molluscan Faunas, in Clark B. L. \& Durham J. W. (eds), Eocene Faunas from the Department of Bolivar, Colombia. Geological Society of America, Memoir 16: 4-126, pl. 1-28. https://doi.org/10.1130/MEM16-p1 CONRAD T. A. 1857. - Descriptions of Cretaceous and Tertiary Fossils. Report on the United States and Mexican Boundary Survey 1 (2): 141-174, pls 1-21. https://www.biodiversitylibrary. org/page/12243303

CORNET F.-L. \& BRIART A. 1866. - On the discovery, in Hainaut, of a Limestone with a Tertiary Fauna, beneath the Sands referred by Professor Dumont to the Landenien System. The Geological Magazine 3 (22): 174, 175. https://www.biodiversitylibrary. org/page/30552012

Cossmann M. 1889. - Catalogue illustré des coquilles fossiles de l'Éocène des environs de Paris faisant suite aux travaux paléontologiques de G.-P. Deshayes. 4 ème fascicule. Annales de la Société royale Malacologique de Belgique 24: 1-385, pls 1-12 [également publié par Cossmann M. 1889: Catalogue illustré des coquilles fossiles de l'Éocène des environs de Paris faisant suite aux travaux paléontologiques de G.-P. Deshayes. 4 ème fascicule. Société royale Malacologique de Belgique, Bruxelles, 385 p., 12 pls.] https://www.biodiversitylibrary.org/page/5502754

COSSMANN M. 1893. - Notes complémentaires sur la faune éocènique de l'Alabama. Annales de Géologie et de Paléontologie 12: 3-51, pls 1-2.

Cossmann M. 1896. - Mollusques éocèniques de la Loireinférieure, tome 1, fascicule 2. Bulletin de la Société des Sciences naturelles de l'Ouest de la France 6 (4): 179-246, pls 4-9 (31 décembre). [également publié par Cossmann M. 1897 : Mollusques éocèniques de la Loire-inférieure. Tome 1, fascicule 2. Muséum d'Histoire naturelle, Nantes: 43-110, 6 pls]. https:// gallica.bnf.fr/ark:/12148/bpt6k112505d/f239.item

Cossmann M. 1899. - Essais de Paléoconchologie comparée. 3ème livraison. l'Auteur \& Comptoir géologique, Paris, 201 p., 8 pls, 35 text-figs. https://www.biodiversitylibrary.org/ page/36853285

Cossmann M. 1913. - Appendice ${ }^{\circ} 5$ au Catalogue illustré des coquilles fossiles de l'Éocène des environs de Paris. Annales de la Société royale zoologique et malacologique de Belgique 49: 19-238, pl. 1-8, text-figs. 1-154 [également publié par CossMANN M. 1913: Catalogue illustré des coquilles fossiles de l'Éocène des environs de Paris faisant suite aux travaux paléontologiques de G.-P. Deshayes. Appendice n ${ }^{\circ}$. Société royale Malacologique de Belgique, Bruxelles, 224 p., 8 pls, 154 text-figs.] https:// www.biodiversitylibrary.org/page/5610647

Cossmann M. 1923. — Description des Mollusques, in O'Gorman G. (ed.), Le gisement cuisien de Gan (Basses-Pyrénées). L'auteur, Pau, 188 p., 14 pls.

Cossmann M. \& Pissarro G. 1901. - Faune éocènique du Cotentin. 2ème article. Bulletin de la Société géologique de Normandie 20: 11-90, pls 7-15. https://www.biodiversitylibrary. org/page/44767962

Cossmann M. \& Pissarro G. 1911. - Iconographie complète des coquilles fossiles de l'Éocène des environs de Paris. Tome 2. Hermann, Paris, pls 26-45. https://doi.org/10.5962/bhl. title. 52315

COTTA B. von 1871. - Geologische Bilder. Leipzig, Weber, 340 p. Courville P., Pacaud J.-M., Merle D. \& Lebrun P. 2012. Le Lutétien de Damery (Marne, France) géologie, environnements, associations de bivalves et gastéropodes. Fossiles. Revue française de Paléontologie hors-série n 3: 57-71, fig. 1-2, pl. 1-11. CROCQ J. 1880. - Liste des fossiles du Bruxellien de WoluweSaint-Lambert et d'Auderghem (Belgique). Annales de la Société malacologique de Belgique 15: 496-498. https://www. biodiversitylibrary.org/page/47414582 
Cuvillier J. 1933. - Nouvelle contribution à la paléontologie du Nummulitique égyptien. Mémoires de l'Institut d'Égypte 22: i-viii + 1-76, pl. 1-8.

DEFRANCE J. L. M. 1817. - Dictionnaire des Sciences naturelles. Tome 5, supplément (Boa-Byw). Levrault, Strasbourg: 10-114 (Buccin, Buccinum fossiles). https://www.biodiversitylibrary. org/page/23001028

De Gregorio A. 1890. - Monographie de la faune éocènique de l'Alabama et surtout de celle de Claiborne de l'étage parisien (Horizon à Venericardia planicosta Lamk). Annales de Géologie et de Paléontologie 7: 1-316, pls 1-46. https://www. biodiversitylibrary.org/page/37164070

Delvaux E. 1884. - Compte-rendu de l'exploration du 15 août aux tranchées de la ligne de Renaix à Lessines. Mémoires de la Société royale ralacologique de Belgique 19: 63-84. https:// www.biodiversitylibrary.org/page/41174566

DeshaYes G.-P. 1832. — Encyclopédie méthodique. Histoire naturelle des Vers. Tome 3. Veuve Agasse, Paris: 595-1152. https://www. biodiversitylibrary.org/page/8899528

Deshayes G.-P. 1835. - Description des coquilles fossiles des environs de Paris. Tome 2. Livraisons 40-45. L'auteur, Béchet Jeune, Baudouin frères, Treuttel \& Wurtz, Paris: 499-780; Atlas 2. Levrault, Paris, pls 79-106. https://doi.org/10.5962/ bhl.title. 52303

Deshayes G.-P. 1844. - Histoire des Mollusques, in DeshAYES G.-P. \& Milne Edwards H. (eds), Histoire naturelle des animaux sans vertèbres, présentant les caractères généraux et particuliers de ces animaux, leur distribution, leurs classes, leurs familles, leurs genres, et la citation des principales espèces qui s'y rapportent; précédée d'une introduction offrant la Détermination des caractères essentiels de l'Animal, sa Distinction du végétal et des autres corps; enfin, l'Exposition des principes Fondamentaux de la Zoologie par J.B.P.A. de Lamarck. Deuxième édition revue et augmentée de notes exposants les faits nouveaux dont la science s'est enrichie jusqu'à ce jour. Tome 10. Baillière, Paris, 639 p. https://doi.org/10.5962/bhl.title.63986

DeshaYes G.-P. 1853. - Traité élémentaire de conchyliologie avec les applications de cette science à la géologie. Explications des planches. Masson, Paris: 49-80. https://doi.org/10.5962/bhl.title.125546

Deshayes G.-P. 1865. - Description des Animaux sans vertèbres découverts dans le bassin de Paris pour servir de supplément à la description des coquilles fossiles des environs de Paris, comprenant une revue générale de toutes les espèces actuellement connues. Tome 3 . Baillière, Paris, Livraisons 45-46, p. 01-288; Atlas 2, pl. 86-93 [2 janvier]; Livraisons 47-48, p. 89-424, Atlas 2, pl. 94-100 [10 juin] ; Livraisons 49-50, p. 425-668; Atlas 2, pl. 101-107 [15 décembre]. https://doi.org/10.5962/bhl.title.51674

Dewalque G. 1865. - Sur la découverte, dans le Hainaut, d'un calcaire grossier avec faune tertiaire, en dessous des sables rapportés par Dumont au système landénien; par mm. Al. Briart $\&$ F.-.L. Cornet, ingénieurs civils. Bulletins de l'Académie royale des Sciences, des Lettres et des Beaux-arts de Belgique, série 2, 20: 721-727. https:/www.biodiversitylibrary.org/page/36021033

Dewalque G. 1866. - Découverte dans le Hainaut par mm. Al. Briart \& F.-.L. Cornet, ingénieurs civils, d'un calcaire grossier avec faune tertiaire. L'Institut, journal universel des Sciences et des Sociétés savantes en France et à l'étranger 1678: 70-72.

DEWALQUE G. 1868. - Prodrome d'une description géologique de la Belgique. Carmanne, Liège, 442 p.

DiLLWYN L. W. 1817. - A Descriptive Catalogue of Recent Shells: Arranged According to the Linnean Method; with Particular Attention to the Synonymy. Volume 1. J. \& A. Arch, London, 580 p. https://doi.org/10.5962/bhl.title.10437

Dulai A., NÓNAY F. \& SZABÓ M. 2017. — Kivételes megtartású középső-eocén (lutetiai) fauna a Párizsi-medencéből (Damery) [Exceptional Middle Eocene (Lutetian) fauna from the Paris Basin (Damery)]. Annales Musei Hitorico-Naturalis Hungarici 109: 147-170, fig. 1-222.
Eaton A. 1826. - Zoological Text-Book Comprising Cuvier's Four Grand Divisions of Animals: also, Shaw's Improved Linnean Genera, Arranged According to the Classes and Orders of Cuvier and Latreille. Short Descriptions of some of the most Common Species are Given for Student's Exercises. Prepared for Rensselaer School and the Popular Class-Room. Websters \& Skinners, Albany, 288 p. https://doi.org/10.5962/bhl.title. 25914

EDWARDS F. E. 1854. - List of the fossil shells from the Eocene strata of Bracklesham Bay, Sussex. Appendix, in PRESTWICH J. (ed.), On the Distinctive Physical and Palaeontological Features on the London Clay and the Bracklesham Sands; and on the Independence of these two groups of Strata. The Quarterly Journal of the Geological Society of London 10 (1-2): 450-454.

EICHWALD E. 1853. - Lethaea Rossica ou Paléontologie de la Russie, Troisième Volume. Dernière période. Schweizerbart, Stuttgart, $\mathrm{xix}+533 \mathrm{p}$.

Favanne De Montcervelle J. G. DE 1780. — La Conchyliologie, ou Histoire naturelle des coquilles de mer, d'eau douce, terrestres et fossiles, avec un traité de la zoomorphose, ou représentation des animaux qui les habitent: ouvrage dans lequel on trouve une nouvelle méthode de les diviser par M. A.J. Dezallier d'Argenville. Troisième édition. De Bure, Paris, Tome 1, Lx + 878 p., Tome 2, 848 p., Tome 3, Atlas, 80 pls. https://doi.org/10.5962/bhl.title.35757

FaVAnne De Montcervelle J. G. DE 1784. - Catalogue systématique et raisonné ou Description du magnifique cabinet appartenant ci-devant à Mr le C. de ***. Quillau, Paris, 558 p., 9 pls. https:// doi.org/10.5962/bhl.title.39137

FÉrUSSAC A. E. J. P. J. F. \& DAUDEBARD DE 1822. - Dictionnaire classique d'Histoire naturelle. Tome 2 (ASA-CAC). Rey \& Gravier, Baudoin, Paris: 550-557 (Buccin, Buccinum). https://gallica.bnf. fr/ark:/12148/bpt6k73032/f558.item

Feugueur L. 1963. - L'Yprésien du Bassin de Paris. Essai de monographie stratigraphique. Imprimerie nationale, Paris, 568 p., 107 text-fig., 8 pls.

FISCHER J.-C. 1980. - Fossiles de France et des régions limitrophes, 2 ème édition. Masson, Paris, 444 p., 195 pls.

FisCHER J.-C. 2000. - Guide des fossiles de France et des régions limitrophes, 3 ème édition. Dunod, Paris, 484 p., 216 pls.

Fischer P. 1883. - Manuel de Conchyliologie et de Paléontologie conchyliologique ou Histoire naturelle des Mollusques vivants et fossiles suivi d'un appendice sur les Brachiopodes par D. Oehlert. Savy, Paris, Fascicule 6: 513-608. https://doi.org/10.5962/bhl.title.13213

FRITEL P. H. 1886. - Fossiles caractéristiques des terrains sédimentaires. Fossiles tertiaires. Savy, Paris, 12 pls.

Fritel P. H. 1903. - Histoire naturelle de la France. 24 partie: Paléontologie (Animaux fossiles). Deyrolle, Paris, 379 p., 445 text-fig., 27 pls.

FurON R. \& SOYER R. 1947. - Catalogue des fossiles tertiaires du bassin de Paris. Lechevallier, Paris, 240 p., 32 pls.

GARVIE C. L. 1996. - The molluscan macrofauna of the Recklaw Formation, Marquez Member (Eocene: Lower Claibornian) in Texas. Bulletin of American Paleontology 111 (352): 1-177, pl. 1-23. https://www.biodiversitylibrary.org/page/28859824

GEINITZ H. B. VON 1845. - Grundriss der Versteinerungskunde. Dresden und Leipzig, Arnoldischen Buchhandlung, $813+2$ p. https://doi.org/10.5962/bhl.title.69285

GerVILLE C. DuHÉRISSIER DE 1817. - Seconde lettre à M. Defrance, sur les coquilles fossiles. Journal de Physique, de Chimie, d'Histoire naturelle et des Arts 84: 197-215. https://gallica.bnf.fr/ark:/12148/ bpt6k96049226/f25.item

GeYs J. F. \& MARQUET R. 1983. - Veldatlas voor Cenozoische Fossielen van België. Deel 2. Het Paleogeen/Fieldatlas on Cenozoic fossils of Belgium. Part. 2: The Paleogene/Atlas de terrain des fossiles cénozoïques belges. Tome 2. Publicatie van de Belgische Vereniging voor Paleontologie 3: 3-203, pls 1-79.

Glibert M. 1933. - Monographie de la Faune malacologique du Bruxellien des environs de Bruxelles. Mémoires du Musée royal d'Histoire naturelle de Belgique 53: 1-214, pl. 1-11. 
GLIBERT M. 1938. - Faune malacologique des sables de Wemmel. 2: Gastropodes, Scaphopodes, Céphalopodes. Mémoires du Musée royal d'Histoire naturelle de Belgique 85: 3-191, pl. 1-4.

GLiBERT M. 1960. — Les Volutacea fossiles du Cénozoïque étranger des collections de l'Institut royal des Sciences Naturelles de Belgique. Mémoires de l'Institut royal des Sciences naturelles de Belgique 2 (61): 1-109.

GliberT M. 1985. — Les Bivalves et Gastéropodes du Bruxellien inférieur de la Belgique (Éocène moyen). Annales de la Société royale malacologique de Belgique 115 (supplément 1): 261-366, pls 1-5.

GMELIN J. F. 1791. — Caroli a Linné [... S Systema nature per regna tria nature: secundum classes, ordines, genera, species, cum characteribus, differentiis, synonymis, locis. Editio XIII, aucta reformata. Classis VI: Vermes. Lipsiæ, Tome 1, pars 6: 3021-3910.

Graves L. 1847. — Essai sur la Topographie géognostique du département de l'Oise. Desjardins, Beauvais, $804 \mathrm{p}$.

Hedley C. \& Pilsbry H. A. 1912. - Strange names for old acquaintances. The Nautilus 26 (4): 45-47.

HermanN J. 1781. - Erste brief über einige Petrefakten an den herausgeben. Der Naturforscher 16: 50-56, pl. 2.

HERMANN J. 1804. - Observationes zoologicae quibus novae complures, alliaeque animalium species describuntur et illustrantur. Opus posthumum edidit Fredericus Ludovicus Hammer. Koenig, Argentorati [Strasbourg] et Parisiis, 332 p. https://doi.org/10.5962/ bhl.title. 13456

Hessel P. 2016. — Stichting Schepsel Schelpfrom. 1991-2016. Part. 1. (Foundation Creature Shell). Stichting Schepsel Schelp, Rhenen, $296 \mathrm{p}$.

HuOT J. J. N. 1824. — Observations sur le banc de Grignon, sur le Calcaire renfermant des restes de végétaux, et sur les couches supérieures de cette localité. Annales des Sciences naturelles 3: 5-15, pl. 1. https://www.biodiversitylibrary.org/page/5813408

INTERNATIONAL COMMISSION ON ZOOLOGICAL NOMENCLATURE 1999 - International Code of Zoological Nomenclature (Fourth edition). The Natural History Museum. Cromwell Road, London, 306 p.

ISENSEE E. 1833. - Elementa nova geographiae et statistices medicinalis. Typis G. Reimeri, 160 p.

JEFFREYS J. G. 1867. - British Conchology or, an Account of the Mollusca which now Inhabit the British Isles and the Surrounding Seas. Vol. 4. John van Voorst, London, 486 p., 8 pls. https://doi. org/10.5962/bhl.title.16342

KeEn A. M. \& BenTSON H. 1944. - Check List of California Tertiary Marine Mollusca. Geological Society of America Special Paper 56: 1-248. https://doi.org/10.1130/SPE56

KNORR G. W. 1764. - Les délices des yeux et de l'esprit, ou collection générale des différentes espèces de Coquillages que la mer renferme, communiquées au public par les héritiers de Georges Wolfgang Knorr. George Wolfgang Knorr, Nürnberg, 52 p., 30 pls. https://doi. org/10.5962/bhl.title.39133

KNORR G. W. 1768a. - Vergnügen der Augen und des Gemüths, in Vorstellung einer allgemeinen Sammlung von Muscheln und andern Geschöpfen, welche im Meer gefunden warden, Dritter Theil. Georg Wolfgang Knorr, Nürnberg. 52 p., 30 pls.

KNORR G. W. 1768b. - Les délices des yeux et de l'esprit, ou collection générale des différentes espèces de Coquillages que la mer renferme, communiquées au public par les héritiers de Georges Wolfgang Knorn, 3ème partie. George Wolfgang Knorr, Nürnberg, 55 p., 30 pls. https://doi.org/10.5962/bhl.title.39133

KNORR G. W. 1772. - Verlustiging der oogen en van den geest, of verzameling van allerley bekende Hoorens en Schulpen, die in haar eigen kleuren afgebeeld zyn. Derde deel. de Erven van F. Houttuyn, Amsterdam: 83-118, 30 pls. https://www.biodiversitylibrary.org/ page/ 13814746

KOKEN E. 1896. - Die Leitfossilien, ein Handbuch für den Unterricht und für das Bestimmen von Versteinerungen. Tauchnitz, Leipzig, 848 p., 256 figs. https://doi.org/10.5962/bhl.title.11861

KOROBKOV I. A. 1955. - Spravochnik I methodicheskoe revodstvo po tretichnym molliuskam. Briuckhonologie [Reference and methodo- logical guide to Tertiary mollusks. Gastropoda]. Gostoptekizdat, Moscou, 795 p., 261 text-figs, 117 pls, 18 tableaux (en Russe).

LAMARCK J.-B. 1803. - Mémoires sur les fossiles des environs de Paris (suite 1). Annales du Muséum d'Histoire naturelle 2 (2): 163-169. https://www.biodiversitylibrary.org/page/3496475

LAMARCK J.-B. 1811. - Mémoires sur les fossiles des environs de Paris. Suite de la détermination des espèces de Mollusques Testacés. Révision du genre Voluta. Annales du Muséum d'Histoire Naturelle 17 (97): 54-80. https://www.biodiversitylibrary.org/page/29406851

LAMARCK J.-B. 1822. - Histoire naturelle des Animaux sans vertèbres présentant les caractères généraux et particuliers de ces Animaux, leur distribution, leurs classes, leurs familles, leurs genres, et la citation des principales espèces qui s'y rapportent et Supplément à divers types de gastéropodes et de Trachélipodes comprenant l'indication des coquilles fossiles qui ne furent point citées dans leurs genres respectifs, Tome 7. L'Auteur, Paris, 711 p. https://doi.org/10.5962/bhl.title.63986

LAUTH T. 1801. - Vitam Johannis Hermann. Argentorati, Typis fratrum Levrault, $64 \mathrm{p}$

Le Renard J. \& PACAud J.-M. 1995. - Révision des Mollusques paléogènes du Bassin de Paris. II - Liste des références primaires des espèces. Cossmanniana 3 (3): 65-132.

LesCure J., Bour R. \& InEICH I. 2009. - Jean Hermann (17381800), Professeur d'histoire naturelle et Herpétologiste strasbourgeois. Bulletin de la Société herpétologique de France 130-131: 1-21

Lhomme L. 1904. - Coquilles fossiles trouvées en 1903 dans les sables de Saint-Gobain (Yprésien). La Feuille des jeunes Naturalistes, 4ème série, 401: 103-106. https://www.biodiversitylibrary. org/page/44464180

LYELL C. 1852. - On the tertiary strata of Belgium and French Flanders. Part II. The Lower Tertiaries of Belgium. The Quarterly Journal of the Geological Society of London 8 (1-2): 277-370, pls 17-20. https://www.biodiversitylibrary.org/page/35461331

MaKarenko D. E. 1976. - Gastropody niznego Paleotsen Severnoj Ukrainy [Gastropods of Paleocene of the North of Ukrainy]. Akademija Nauk Ukrainskoj S.S.R., Institut Geologiceskij Nauk, Kiev, 180 p., 18 pls. [En Russe].

MarTini F. H. W. 1777. - Neues systematisches Conchylien-Cabinet. Nach der Natur gezeichnet und mit lebendigen Farben erleuchtet. III. Band. Raspe, Nürnberg, p. [vi] + vi + 434, pl. LXVI-CXXI [= 66-121]. https://doi.org/10.5962/bhl.title.43760

MAYER-EYMAR C. 1876. - Systematisches Verzeichniss der Versteinerungen des Parisian der Umgegend von Einsiedeln. Caesar Schmidt, Zürich, 100 p., 4 pls. (également publié par Mayer-Eymar C. 1877: Paläontologie der Pariserstufe von Einsiedeln und seinen Umgebungen. Beiträge zur Geologischen Karte der Schweiz 14 [2]: 1-100, pl. 1-4).

MAYER-EYMAR C. 1886. — Zur Geologie Egyptens. Vierteljahrsschrift der Naturforschenden Gesellschaft in Zürich 31: 241-267. https://www.biodiversitylibrary.org/page/8705892

Melleville M. 1837. - Liste des espèces fossiles des environs de Laon. Bulletin de la Société géologique de France 8: 248-249.

MELLEVILLE M. 1843. - Mémoire sur les sables tertiaires inférieurs $d u$ bassin de Paris, avec la description de 78 espèces de coquilles fossiles inédites de ce terrain, et dix planches représentant ces espèces. Fortin, Masson et Cie, Paris, 88 p., 9 pls. [Également publié Melleville M. 1843: Mémoire sur les sables tertiaires inférieurs du bassin de Paris, avec la description de 78 espèces de coquilles fossiles inédites de ce terrain, et dix planches représentant ces espèces. Annales des Sciences géologiques 2: 1-29 et 77-120, pl. 1-9].

MelleVille M. 1857. - Composition géologique de la Montagne de Laon. Bulletin de la Société académique de Laon 6: 89-111. https://gallica.bnf.fr/ark:/12148/bpt6k4412045/f113.item

Merle D. 1986. - Contribution à l'étude paléontologique du gisement de Gan (Pyrénées-Atlantiques): systématique, évolution et paléoécologie. Diplôme de l'EPHE, Dijon, 394 p.

Merle D. 1989. - Étude des Gastéropodes, in CheVAluIER M., Barreau A., Chateauneuf J. J., Courboulex S., Esteoule-Choux J., Farjanel G. Genot P., Hollier-Larousse A., Margerel J.-P., Merle D., Ollivier-Pierre M.-F., Lauriat-Rage A. \& 
Trauth N. (eds), Le couloir «le Maupas - les Étangs» : aspects structurels, sédimentologiques et paléontologiques (La Limouzinière, Loire-Atlantique). Géologie de la France 1-2: 79-102, 9 figs, 5 tableaux.

Merle D. \& PaCaud J.-M. 2003. - New species of Eocithara Fischer, 1883 (Mollusca, Gastropoda, Harpidae) from the Early Paleogene with phylogenetic analysis of the Harpidae. Geodiversitas 26 (1): 61-87.

Merle D., Pacaud, J.-M., Kriloff A. \& Loubry P. 2008. — Les motifs colorés résiduels des coquilles lutétiennes du bassin de Paris, in MerLe D. (ed.), Stratotype Lutétien. Muséum national d'Histoire naturelle, Paris; Biotope, Mèze; BRGM, Orléans: 82-227, pls 18-35 (Patrimoine géologique; 1 ).

Meunier S. 1875. - Géologie des environs de Paris ou Description des terrains et énumération des fossiles qui s'y rencontrent. Baillière, Paris, $510 \mathrm{p}$

Meunier S. 1912. - Géologie des environs de Paris: description des terrains et énumération des fossiles qui s'y rencontrent, suivie d'un index des localités fossiliferes. Baillière, Paris, $540 \mathrm{p}$.

Mörch O. A. L. 1858. - Note sur le genre Volutharpa. Journal de Conchyliologie 7 (1): 40-44. https://www.biodiversitylibrary. org/page/15863013

MORLEY DAVIES A. 1935. - Tertiary Fauna. A Text-book for Oilfield Palaeontologists and Students of Geology. Vol. 1. The composition of Tertiary Faunas. Thomas Murby \& Co, London, $\mathrm{x}+406$ p. 568 figs.

MORRIS J. 1854. - A Catalogue of British Fossils: Comprising the Genera and Species Hitherto Described; with References to their Geological Distribution and to the Localities in which they have been found. Second Edition. The Author, London, viii $+372 \mathrm{p}$.

Murchison R. I., De Verneuil E. \& vOn Keyserling C. A. 1845. - The Geology of Russia in Europe and the Ural Mountains, Vol. 1. Geology. John Murray, London, xvi +700 p.

NeLSON R. N. 1925. - A contribution to the paleontology of the Martinez Eocene of California. University of California Publications in Geological Sciences 15 (11): 397-466.

NeWTON R. B. 1891. - Systematic List of the Frederick E. Edwards Collection of British Oligocene and Eocene Mollusca in the British Museum (Natural History), with References to the Type Specimens from Similar Horizons Contained in other Collections Belonging to the Geological Department of the Museum. British Museum (Natural History), London, xxviii + 365 p. https://doi.org/10.5962/bhl.title.24285

NysT P. H. 1845. - Description des coquilles et des polypiers fossiles des terrains tertiaires de la Belgique. Mémoire en réponse à la question suivante: faire la description des coquilles et des polypiers fossiles des terrains tertiaires de la Belgique et donner l'indication précise des localités et des systèmes de roches dans lesquels ils se trouvent. Hayez, Bruxelles, 675 p., 48 pls. [Également publié par Nyst P. H. 1845 : Description des coquilles et des polypiers fossiles des terrains tertiaires de la Belgique. Mémoire en réponse à la question suivante: faire la description des coquilles et des polypiers fossiles des terrains tertiaires de la Belgique et donner l'indication précise des localités et des systèmes de roches dans lesquels ils se trouvent. Mémoires couronnés et Mémoires des Savants Étrangers de l'Académie royale des Sciences et des Belles-lettres de Bruxelles 17: 1-697, pl. 1-15]. https://www.biodiversitylibrary. org/page/2668665

Nyst P. H. 1853. - Liste des fossiles des sables calcarifères de Bruxelles, in D’Omalius D’Halloy J. J. (ed.), Abrégé de Géologie. Jamar, Bruxelles: 579-581.

OLSSON A. A. 1967. - Some Tertiary Molusks from South Florida and the Caribbean. Paleontological Research Institution, Ithaca, New York, $61 \mathrm{p}$.

[OPINION 184]. 1944. - On the statut of names first published in volumes 1 to 11 of Martini (F. H. W.) and Chemnitz (J. H.), Neues systematisches Conchylien-Cabinet, Nürnberg, 17691795. Opinions and declarations rendered by the International Commission on Zoological Nomenclature 3 (3): 25-36.
Orbigny A. D’ 1850. - Prodrome de Paléontologie stratigraphique universelle des Animaux mollusques et rayonnés faisant suite au cours élémentaire de Paléontologie et de géologie stratigraphiques. Vol. 2. Masson, Paris, 428 p. https://doi.org/10.5962/bhl.title.45605

OrtLieb J. \& ChELlonNeIX E. 1870. —Étude géologique des collines tertiaires du département du Nord comparées avec celles de la Belgique. Mémoires de la Société des Sciences, de l'Agriculture et des Arts de Lilles 3 (8): 109-336, pls 1-7. (également publié par Ortlieb J. \& ChellonNeix E. 1870 : Étude géologique des collines tertiaires du département du Nord comparées avec celles de la Belgique. Quarré et Castiaux, Lilles, 216 p., 7 pls).

PaCaud J.-M. 2003. - First fossil records of the Recent Ovulid genus Pseudocypraea Schilder, 1927 (Mollusca: Gastropoda) with description of a new species. Geodiversitas 25 (3): 451-462, text-fig. 1-6.

PACAUD J.-M. 2008. - Le contenu paléontologique du Lutétien du bassin de Paris: Mollusca, in MerLe D. (éd.), Stratotype Lutétien. Muséum national d'Histoire naturelle, Paris; Biotope, Mèze; BRGM, Orléans: 40-95. (Patrimoine géologique; 1).

Pacaud J.-M. 2016. - Découverte du matériel type de Niso eburnea Risso, 1826, espèce type du genre Niso Risso, 1826 et implications taxonomiques. Bulletin de la Société géologique de France 187 (1): 55-62, fig. 1-2, pl. 1. https://doi.org/10.2113/ gssgfbull.187.1.55

PACAUD J.-M. 2017a. - Nouvelle observation du motif résiduel de couleur préservé chez Athleta (Volutocorbis) suturalis (Nyst, 1836) (Mollusca, Gastropoda, Volutidae) des sables priaboniens (Éocène supérieur) de Dnipro (Oblast de Dnipropetrovsk, Ukraine). Міжнародна науково-технінна конференція. Розвиток Промисловості Та Суспільства: 73-75, fig. 1a, b.

PACAUD J.-M. 2017b. - Новые Аанные Об Окраске, Сохранившейся На Раковинах Athleta (Volutocorbis) suturalis (Nyst, 1836) (Mollusca, Gastropoda, Volutidae) Из Приабона (Верхний Эоцен) Г. Анепра (Анепропетровская Область, Украина). Міжнародна науково-технічна конференціл. Розвиток Промисловості Та Суспільства, Секція 5, Геологія іприкладна мінералогія: 76-77, fig. $1 \mathrm{a}, \mathrm{b}$.

PACAUD J.-M. 2017c. - Trahaldia, un nouveau genre de Columbellidae (Gastropoda: Neogastropoda) de l'Éocène moyen du bassin de Paris. Xenophora Taxonomy 16: 3-8, fig. 1-2, pl. 1-2.

PaCAud J.-M. 2018. - Les Cypraeoidea (Mollusca, Caenogastropoda) du Priabonien (Éocène supérieur) de Dnipro (Oblast de Dnipropetrovsk, Ukraine). Partie 2: Ovulidae (le genre Projenneria Dolin, 1997). Xenophora Taxonomy 21: 11-21, fig. 1-2, pl. 1-3.

PaCAUd J.-M. \& Le Renard J. 1995. - Révision des Mollusques paléogènes du bassin de Paris. 4: Liste systématique actualisée. Cossmanniana 3 (4): 151-187.

PACAUd J.-M. \& CAZE B. 2011. - Une espèce nouvelle de Rostellariidae (Mollusca, Gastropoda, Littorinimorpha) dans le Lutétien (Éocène moyen) du bassin de Paris. Redécouverte du matériel type de Hippochrenes fissura (Coquebert de Montbret \& Brongniart, 1793). Cossmanniana 13: 21-31, pl. 1-4.

PaCAud J.-M. \& PONS J. 2013. - Contribution des motifs résiduels de couleur dans la discrimination des espèces d'Athletinae (Mollusca: Gastropoda) de l'Éocène du bassin de Paris. Fossiles. Revue française de paléontologie 16: 21-39, pl. 1-8, text-fig. 1-6.

PACAUD J.-M. \& CAZES L. 2014. — Motif coloré résiduel préservé sur des coquilles du genre Bullia Gray in Griffith \& Pidgeon, 1833 (Mollusca: Gastropoda) de l'Éocène moyen du bassin de Paris et des Etats-Unis. Xenophora 147: 16-22, text-fig. 1-2, pl. 1-2.

PaCaud J.-M. \& Ledon D. 2014. - Révision des Mollusques de l'Éocène de Blaye (Gironde). 4. Description d'une espèce nouvelle de Pachycrommium (Gastropoda, Caenogastropoda, Ampullinidae). Notes taxonomiques et nomenclaturales sur quelques Ampullinidae avec la description de trois espèces nouvelles. Bulletin de la Société Linnéenne de Bordeaux 149, 42 (2): 183-198, pl. 1-7. 
PACAUD J.-M. \& LAPORTE J. 2015. — Première occurrence de l'espèce du Rupélien (Oligocène inférieur) de Belgique Athleta (Volutocorbis) suturalis (Nyst, 1836) dans les sables bartoniens (Éocène moyen) du bassin de Paris. Xenophora 151: 18-24, text-fig. 1-3.

PACAUD J.-M. \& ViCián Z. 2019. - Description of a new species of Subepona Dolin \& Lozouet, 2004 (Mollusca: Cypraeoidea) from the Lutetian (Middle Eocene) of Hungary. Fragmenta Palaeontologica Hungarica 35: 103-110, fig. 1-15. https://doi. org/10.17111/FragmPalHung.2018.35.103

PaCAUd J.-M., Ledon D. \& Loubry P. 2015. — Les Plesiotritoninae (Mollusca: Gastropoda: Cancellariidae) de l'Éocène du bassin de Paris, du Cotentin, de Loire-Atlantique et d'Aquitaine. Palaeontos 27: 65-119, text-fig. 1-6, tableaux 1-6, pl. 1-12.

Palmer K. VAn Winkle 1937. - The Claibornian Scaphopoda, Gastropoda and Dibranchiate Cephalopoda of the Southern United States. Bulletins of American Paleontology 7 (32): 1-548, pl. 1-90.

Palmer K. VAN WinkLe 1977. - The Unpublished Vélins of Lamarck (1802-1809). Illustrations of Fossils of the Paris Basin Eocene. Paleontological Research Institution, Ithaca, 67 p., 52 pls.

PARKINSON J. 1811. — Organic Remains of a Former World: an Examination of the MiNeralized Remains of the Vegetables and Animals of the Antediluvian World; Generally Termed Extraneous Fossils. Volume 3. Sherwood, Neely and Jones, London, 484 p., 22 pls. https://doi.org/10.5962/bhl.title.30613

PARKINSON J. 1833. - Organic Remains of a Former World: an Examination of the Mineralized Remains of the Vegetables and Animals of the Antediluvian World; Generally Termed Extraneous Fossils. Volume 3. Second edition. Nattali, London, 467 p., 22 pls.

PeZANT A. 1910. - Coquilles fossiles des calcaires grossiers de Parnes, 1890-1910. La Feuille des Jeunes Naturalistes 4 (480): 185-197, pl. 13-14. https://www.biodiversitylibrary.org/page/34523239

PfeIfFER L. 1840. - Kritisches Register zu Martini und Chemnitz' Systematischem Konchylien-Kabinet. Th. Fischer, Cassel, 112 p. https://doi.org/10.5962/bhl.title.37917

PICTET F. J. 1857. - Traité de paléontologie: ou Histoire naturelle des animaux fossiles considérés dans leurs rapports zoologiques et géologiques, Atlas. Baillière \& Fils, Paris, 77 p., 110 pls. https:// doi.org/10.5962/bhl.title.13903

Plaziat J.-C. 2011. - Bernard Palissy (1510-1590) and the French geologists: a critical reappraisal concerning the founding naturalist and his rustic ceramics. Bulletin de la Société géologique de France 182 (3): 255-267.

Plaziat J.-C. 2019. — L'identification des moulages des coquilles fossiles et des organismes actuels des "rustiques figulines": un apport naturaliste à la caractérisation des ateliers successifs de Palissy et de ses émules. Technè 47: 102-115. https://doi. org/10.4000/techne.1569

Pomerol C. \& Feugueur L. 1974. - Bassin de Paris, Ile de France, Pays de Bray. 2eme édition entièrement refondue. Guides géologiques régionaux, Masson, Paris, 216 p., 27 pls.

PRESTWICH J. 1856. - On the correlation of the Eocene Tertiarie of England, France and Belgium, Part. 2. The Quarterly Journal of the Geological Society of London 13 (1): 89-134. https://www. biodiversitylibrary.org/page/35315466

Pusch G. Q. 1837. — Polens Paläontologie, oder Abbildung und Beschreibung der vorzüglischten und der noch unbeschriebenen Petrefakten aus den Gebirgs-Formationen in Polen, Wolhynien und dem Karpathen. Schweizerbart's verlagshandlung, Stuttgart, 218 p., 16 pls.

Romé DE L'ISLE J.-B. L. DE 1767. - Catalogue systématique et raisonné des curiosités de la nature et de l'art, qui composent le Cabinet de M. Davila. Tome 3. Briasson, Paris, 286 p. https:// doi.org/10.5962/bhl.title.77519

RoISSY F. DE 1805. - Histoire naturelle, générale et particulière, des mollusques, animaux sans vertèbres et à sang blanc. Faisant suite aux ceuvres de Leclerc de Buffon, et partie du cours rédigé par C. S. Sonnini. Histoire naturelle des Mollusques. Tome 6. Dufart, Paris, 480 p., pls 57-72. https://doi.org/10.5962/bhl.title.51804
SCHMIDT F. A. VON 1846. - Petrefacten-Buch oder allgemeine und besondere Versteinerungskunde: mit Berücksichtigung der Lagerungsverhältnisse, besonders in Deutschland. Hoffmann'sche Verlags-Buchhandlung, Stuttgart, 174 p., 63 pls. https://doi. org/10.5962/bhl.title. 45497

SCHREIBERS K. 1793. - Versuch einer vollständigen Conchylienkenntnißnach Linnes System, Erster Band. Von Kurzbeck, Wien, 446 p. http://mdz-nbn-resolving.de/urn:nbn:de:bvb:12-bsb10707128-6

SCHRÖTER J. S. 1785. — Neue Litteratur und Beyträge zur Kenntniß der Naturgeschichte; vorzüglich der Conchylien und Fossilien. Volume 2, Müller, Leipzig, 632 p., 4 pls.

SEBA A. 1765. - Locupletissimi rerum naturalium thesauri accurata descriptio, et iconibus artificiosissimis expressio, per universam physices historiam. Tome 4. H. C. Arksteum, et H. Merkum, et Petrum Schouten, Amstelaedami, $42+226$ p., 108 pls. https:// doi.org/10.5962/bhl.title. 62760

SOlANDER D. C. 1766. - Descriptiones Specierum, in Brander G. (ed.), Fossilia Hantoniensia collecta, et in Musaeo Brittannico deposita. London: 9-43, 9 pls. https://doi.org/10.5962/bhl.title.146466

SOWERBY G. B. (I) 1825. - The Genera of Recent and Fossil Shells, for the use of students in Conchology and Geology, with original plates, no 25. London, 6 pls. (Genera Nassa, Buccinum, Coronula, Sanguinolaria, Saxicava). https://doi.org/10.5962/bhl.title.86281

SOWERBY G. B.(II) 1842. - A Conchological Manual. Second edition. Henry Bohn, London, vi + 313 p., 27 pls. https://doi. org/10.5962/bhl.title.64833

SOWERBY J. DE C. 1850. - Notes and Descriptions of New Species (Brackelsham Mollusca) - Descriptions of the Fossils from the Eocene Deposits of Bognor (Mollusca) - Figs. of Mollusca, in Dixon F. (éd.), The Geology and Fossils of the Tertiary and Cretaceous Formations of Sussex. Longmans, Brown, Green and Longmans, London: 62-194 et 222-228, pl. 2-9 et 14-15.

SQUiRES R. L. 1984. - Megapaleontology of the Eocene Llajas Formation, Simi Valley, California. Contributions in Science 350: 1-76, fig. 1-19. https://www.biodiversitylibrary.org/page/52130222

STEnzel H. B. \& TuRner F. E. 1940. - The gastropod genera Cryptochorda and Lapparia in the Eocene of the Gulf Coastal Plain. The University of Texas Publication 3945: 795-828, pls 43-45. http://hdl.handle.net/2152/23428

STRAUSZ L. 1966. — Dudari eocén Csigák [Les Gastropodes de l'Éocène de Dudar]. Geologica Hungarica, Series Palaeontologica 33: 1-200, pl. 1-24.

SWAINSON W. 1840. - A Treatise on Malacology: or Natural Classification of Shells and Shell Fish. Longman, Orme, Brown, Green \& Longmans, London, viii + 419 p. https://doi.org/10.5962/bhl. title.8027

Termier H. \& Termier G. 1960. — Paléontologie stratigraphique. Quatrième fascicule: Éocène-Oligocène-Miocène-Pliocène-Quaternaire et index général. Masson, Paris: 359-512.

Tracey S., Todd J. A., Le Renard J., King C. \& Goodchild M. 1996. - Distribution of Mollusca in Units S1 to S9 of the Selsey Formation (middle Lutetian), Selsey Peninsula, West Sussex. Tertiary Research 16 (1-4): 97-139, pl. 1-3.

Traub F. 1981. - Weitere Paleozän-Gastropoden aus dem Helvetikum des Haunsberges nördlich von Salzburg. Mitteilungen der Bayerischen Staatssammlung für Paläontologie und Historische Geologie 21: 41-63, pls 10-11. https://www.biodiversitylibrary. org/page/28728456

Tréguier J., Pacaud J.-M. \& Prugneaux V. 2017. - Des types porte-noms de la collection Defrance conservés au Musée des Sciences de Laval. Fossiles. Revue française de paléontologie 32: 21-34, fig. 1-15.

TURNER F. E. 1938. - Stratigraphy and Mollusca of the Eocene of western Oregon. Geological Society of America, Special Paper 10: i-ix + 1-130, pl. 1-23. https://doi.org/10.1130/SPE10

TrYON G. W. 1883. - Structural and Systematic Conchology: an Introduction to the Study of the Mollusca. Vol. 2. The Author, Philadelphia, 430 p., pls 23-91. https://doi.org/10.5962/bhl.title.10347 
VAN Nieulande F. 2002. - Voorlopig overzicht van de molluskenfauna's in de groeve van de steebakkerij Ampe te Egem (B). Afzettingen WTKG 23 (2): 30-33.

VASSEUR G. 1881. - Recherches géologiques sur les Terrains tertiaires de la France occidentale. Stratigraphie. Première partie - Bretagne. Annales des Sciences géologiques 13: 1-432, 29 figs, 6 cartes (également publié par VASSEUR G. 1881 : Recherches géologiques sur les Terrains tertiaires de la France occidentale. Stratigraphie. Première partie - Bretagne. Bibliothèque de l'École des Hautes Études [section des Sciences naturelles], Masson, Paris, 432 p., 29 figs, 6 cartes). https://gallica.bnf.fr/ark:/12148/bpt6k432589s/f4.item

VOKES H. E. 1939. - Molluscan faunas of the Domengine and Arroyo Honda formations of the California Eocene. Annals of the New York Academy of Sciences 38: 1-246, pls 1-22. https:// doi.org/10.1111/j.1749-6632.1939.tb55368.x

VOKES H. E. \& VOKES E. H. 1968. - Variation in the genus Orthaulax (Mollusca: Gastropoda). Tulane studies in Geology and Paleontology 6 (2): 71-79.

WALCH J. E. E. 1768. - Recueil de monumens des catastrophes que le Globe de le Terre à éssuiées, contenant des pétrifications dessinées, gravées et enluminées, d'après des originaux, commencé par feu $M r$. George Wolfgang Knorr, et continué par ses heritiers avec l'histoire naturelle de ces corps, Tome second, Section 1. Nuremberg, [Édition française], 159 p. https://gallica.bnf.fr/ark:/12148/bpt6k1512374m

WATElET A. 1855. - Catalogue des fossiles observés dans les couches de sable comprises entre les fausses glaises et lignites et le calcaire grossier dans le département de l'Aisne, et principalement dans les environs de Soissons. Bulletin de la Société archéologique, historique et scientifique de Soissons 9: 41-70 (également publié par WATELET A. 1855: Recherches dans les sables tertiaires des environs de Soissons, Fascicule 3. Catalogue de fossiles. Société historique, archéologique et scientifique de Soissons, Laon: 5-34). https://gallica.bnf.fr/ark:/12148/ bpt6k453995m/f39.item

Watelet A. 1870. — Le bassin de Paris. Recueil de Mémoires relatifs au Bassin tertiaire de cette région et à l'époque quaternaire. Catalogue des Mollusques des sables inférieurs. Savy, Paris, 24 p. https:// gallica.bnf.fr/ark:/12148/bpt6k1185295b

WeAver C. E. 1942. - Paleontology of the marine Tertiary formations of Oregon and Washington. Part. 2: Description of species. Mollusca: Gastropoda; Cephalopoda; Arthropoda. University of Washington Publications in Geology 5: 279-562, pls 62-104.

WeNZ W. 1943. - Gastropoda. Teil 6: Prosobranchia (suite), in Handbuch der Palaeozoologie. Band 6. Borntraeger, Berlin: 2011506, text-figs 3417-4211.

WiCKERSHEIMER E. 1921. — Les notes de Jean Hermann sur les cabinets de curiosités qu'il visita à Paris (1763-1764). Compte Rendu de la 44 ìme Session de l'Association française pour l'Avancement des Sciences, Strasbourg. Paris, l'Association \& Masson et Cie: 581-583.

WinkLer T. C. 1868. - Musée Teyler. Catalogue systématique de la collection paléontologique: Premier supplément. Les Heritiers Loosjes, Harlem, 278 p. https://gallica.bnf.fr/ark:/12148/bpt6k880173d

Wrigley A. 1934. - A Lutetian fauna at Southampton Docks. Proceedings of the Geologists' Association 45: 1-16. https://doi. org/10.1016/S0016-7878(34)80030-2

ZitTel K. A. VON 1885. - Handbuch der Palaeontologie. Palaeozoologie. 1. Abteilung, 2. Mollusca und Arthropoda. Oldenburg, München und Leipzig, 893 p. https://doi.org/10.5962/bhl. title.34265 
ANNEXE 1. - Cryptochorda (s.str.) stromboides (Hermann, 1781), liste du matériel étudié (1187 exemplaires). Abréviations: $\mathbf{N}^{\circ}$, numéro du spécimen correspondant dans les collections de Paléontologie du Muséum (MNHN.F); Coll., nom de la collection; Nb spec., nombre de spécimens.

\begin{tabular}{|c|c|c|c|c|c|c|c|c|c|}
\hline No coll. & Coll. & $\begin{array}{l}\mathrm{Nb} \\
\text { spec. }\end{array}$ & Localités & Étages & No coll. & Coll. & $\begin{array}{l}\mathrm{Nb} \\
\text { spec. }\end{array}$ & Localités & Étages \\
\hline $\begin{array}{l}\text { Néotype } \\
\text { A57573 }\end{array}$ & de Roissy & 1 & Courtagnon (Marne) & Lutétien & A57639 & MNHN & 1 & $\begin{array}{l}\text { Ferté/Jouarre (Seine-et- } \\
\text { Marne) }\end{array}$ & Bartonien \\
\hline A57589 & de Roissy & 6 & Courtagnon (Marne) & Lutétien & A57640 & MNHN & 4 & Mary-sur-Marne (Seine- & Bartonien \\
\hline A57770 & de Roissy & 4 & Courtagnon (Marne) & Lutétien & & & & et-Marne) & \\
\hline A57576 & Pacaud & 3 & Damery (Marne) & Lutétien & * & Six & 6 & Caumont (Seine-et- & Bartonien \\
\hline A71408 & Pacaud & 1 & Damery (Marne) & Lutétien & & & & Marne) & \\
\hline A71411 & Pacaud & 1 & Damery (Marne) & Lutétien & A57638 & MNHN & 1 & Crouy (Aisne) & Bartonien \\
\hline A71412 & Pacaud & 1 & Damery (Marne) & Lutétien & A57641 & Pacaud & 2 & Bézu-le-Guéry (Aisne) & Bartonien \\
\hline A57580 & MNHN & 10 & Damery (Marne) & Lutétien & A71467 & Pons & 6 & Bézu-le-Guéry (Aisne) & Bartonien \\
\hline A57581 & MNHN & 24 & Damery (Marne) & Lutétien & A57575 & de Roissy & 103 & Parnes (Oise) & Lutétien \\
\hline A57585 & MNHN & 3 & Damery (Marne) & Lutétien & A71685 & de Roissy & 127 & Parnes (Oise) & Lutétien \\
\hline B65168 & Lhomme & 2 & Damery (Marne) & Lutétien & A71670 & de Roissy & 13 & Parnes (Oise) & Lutétien \\
\hline A11521 & d'Orbigny & 2 & Damery (Marne) & Lutétien & A57577 & de Roissy & 13 & Parnes (Oise) & Lutétien \\
\hline B70689 & d'Orbigny & 7 & Damery (Marne) & Lutétien & A57578 & MNHN & 7 & Parnes, Les Boves & Lutétien \\
\hline A71654 & MNHN & 3 & Damery (Marne) & Lutétien & & & & (Oise) & \\
\hline A57630 & Staadt & 12 & Damery (Marne) & Lutétien & B65181 & Sarazin & 3 & Parnes (Oise) & Lutétien \\
\hline A57631 & Staadt & 24 & Damery (Marne) & Lutétien & A11520 & d'Orbigny & 4 & Parnes (Oise) & Lutétien \\
\hline A71409 & Staadt & 1 & Damery (Marne) & Lutétien & B65185 & MNHN & 1 & Parnes (Oise) & Lutétien \\
\hline A71414 & Staadt & 1 & Damery (Marne) & Lutétien & B65183 & Morlet & 3 & Parnes (Oise) & Lutétien \\
\hline A71415 & Staadt & 1 & Damery (Marne) & Lutétien & B65184 & Lhomme & 2 & Parnes (Oise) & Lutétien \\
\hline B65172 & Morlet & 2 & Damery (Marne) & Lutétien & B65186 & Sarazin & 1 & Parnes, Launay (Oise) & Lutétien \\
\hline B65167 & $1965-11$ & 1 & Damery (Marne) & Lutétien & A57596 & MNHN & 2 & Parnes (Oise) & Lutétien \\
\hline A71655 & Schtrock & 1 & Damery (Marne) & Lutétien & A57626 & MNHN & 2 & Parnes (Oise) & Lutétien \\
\hline A71303 & Crochet & 7 & Damery (Marne) & Lutétien & A57628 & MNHN & 4 & Parnes (Oise) & Lutétien \\
\hline A71469 & Pons & 3 & Damery (Marne) & Lutétien & A71672 & Pacaud & 16 & Parnes, Grande- & Lutétien \\
\hline A71656 & Faullummel & 1 & Damery (Marne) & Lutétien & & & & Crognière (Oise) & \\
\hline A70493 & Hoffstetter & 11 & Damery (Marne) & Lutétien & S10564 & Péron & 4 & Parnes (Oise) & Lutétien \\
\hline * & Vyncke & 9 & Damery (Marne) & Lutétien & * & Boucher & 2 & Parnes (Oise) & Lutétien \\
\hline * & Six & 30 & Damery (Marne) & Lutétien & * & Six & 14 & Monts (Oise) & Lutétien \\
\hline * & Boucher & 4 & Damery (Marne) & Lutétien & A57624 & MNHN & 4 & Mouchy-le-Châtel (Oise) & Lutétien \\
\hline A57579 & MNHN & 6 & Chamery (Marne) & Lutétien & * & Six & 2 & Châteaurouge (Oise) & Lutétien \\
\hline A57587 & Ballot & 2 & Chamery (Marne) & Lutétien & * & $\operatorname{Six}$ & 5 & Ully-Saint-Georges & Lutétien \\
\hline B65176 & de Morgan & 3 & Chamery (Marne) & Lutétien & & & & (Oise) & \\
\hline A57594 & MNHN & 7 & Chamery (Marne) & Lutétien & * & Six & 1 & Ponchon (Oise) & Lutétien \\
\hline A57629 & de Saint- & 3 & Chamery (Marne) & Lutétien & B65179 & Morlet & 2 & Saint-Félix (Oise) & Lutétien \\
\hline & Marceaux & & & & B65180 & $1965-1$ & 1 & Saint-Félix (Oise) & Lutétien \\
\hline A57650 & Péron & 8 & Chamery (Marne) & Lutétien & A71658 & Pacaud & 1 & Chaumont-en-Vexin, & Lutétien \\
\hline * & Six & 5 & Chamery (Marne) & Lutétien & & & & Darcy (Oise) & \\
\hline A57582 & Watelet & 3 & Montmirail (Marne) & Lutétien & A57652 & MNHN & 22 & Chaumont-en-Vexin & Lutétien \\
\hline A57586 & MNHN & 2 & Montmirail (Marne) & Lutétien & & & & (Oise) & \\
\hline A57771 & de Roissy & 2 & Montmirail (Marne) & Lutétien & A71671 & MNHN & 8 & Chaumont-en-Vexin & Lutétien \\
\hline A57595 & MNHN & 7 & Montmirail (Marne) & Lutétien & & & & (Oise) & \\
\hline B65166 & d'Orbigny & 3 & Montmirail (Marne) & Lutétien & 365177 & MNHN & 2 & Chaumont-en-Vexin & Lutétien \\
\hline A57583 & Pacaud & 39 & Fleury-la-Rivière & Lutétien & & & & (Oise) & \\
\hline B65165 & Mortlet & 2 & $\begin{array}{l}\text { (Marne) } \\
\text { Fleury-la-Rivière } \\
\text { (Marne) }\end{array}$ & Lutétien & A71669 & Pacaud & 1 & $\begin{array}{l}\text { Chaumont-en-Vexin } \\
\text { (Oise) }\end{array}$ & Lutétien \\
\hline & & & (Marne) & & * & Six & 1 & Chaumont-en-Vexin & Lutétien \\
\hline * & Six & 10 & $\begin{array}{l}\text { Fleury-la-Rivière } \\
\text { (Marne) }\end{array}$ & Lutétien & & & & (Oise) & \\
\hline * & Boucher & & & & B65187 & Lhomme & 2 & Boury-en-Vexin (Oise) & Lutétien \\
\hline B71754 & $\begin{array}{l}\text { Boucher } \\
\text { MNHN }\end{array}$ & $\begin{array}{l}4 \\
2\end{array}$ & $\begin{array}{l}\text { Venteuil (Arty) (Marne) } \\
\text { Boursault (Marne) }\end{array}$ & Lutétien & A57643 & Pacaud & 5 & Baron (Oise) & Bartonien \\
\hline A57633 & MNHN & $\begin{array}{l}2 \\
1\end{array}$ & & Lutetien & * & Boucher & 3 & Baron (Oise) & Bartonien \\
\hline & Van Hyfte & 3 & $\begin{array}{l}\text { Boursault (Marne) } \\
\text { Nantheuil-la-Fôret }\end{array}$ & Lutetien & * & Ledon & 2 & Barisseuse (Oise) & Bartonien \\
\hline & & 3 & $\begin{array}{l}\text { Nantheuil-la-Fôret } \\
\text { (Marne) }\end{array}$ & Lutetien & * & Boucher & 1 & Barisseuse (Oise) & Bartonien \\
\hline & & & $\begin{array}{l}\text { (Marne) } \\
\text { Nantheuil-la-Fôret }\end{array}$ & & A71679 & Sautereau & 15 & Authevernes (Eure) & Lutétien \\
\hline * & Danvin & 7 & $\begin{array}{l}\text { Nantheuil-la-Fôret } \\
\text { (Marne) }\end{array}$ & Lutétien & * & Sautereau & 130 & Authevernes (Eure) & Lutétien \\
\hline & & & $\begin{array}{c}\text { (Marne) } \\
\text { Mécringes (Marne) }\end{array}$ & & * & Vyncke & 7 & Authevernes (Eure) & Lutétien \\
\hline A71413 & Faullummel & 1 & Mécringes (Marne) & Bartonien & * & Boucher & 18 & Authevernes (Eure) & Lutétien \\
\hline A57636 & Faullummel & 3 & Mécringes (Marne) & Bartonien & A71418 & Pacaud & 1 & Fontenay-en-Vexin & Lutétien \\
\hline A57644 & Pacaud & 1 & Le Limon (Seine-et- & Bartonien & & & & (Eure) & \\
\hline & & & & & A57634 & Pacaud & 1 & Fontenay-en-Vexin & Lutétien \\
\hline * & Six & 1 & Le Limon (Seine-et- & Bartonien & & & & (Eure) & \\
\hline & & & & & A71675 & Pacaud & 19 & Fontenay-en-Vexin & Lutétien \\
\hline * & Boucher & 1 & Le Limon (Seine-et- & Bartonien & & & & (Eure) & \\
\hline & & & & & * & Vyncke & 5 & Fontenay-en-Vexin & Lutétien \\
\hline A71416 & Faullummel & 1 & Dhuisy (Seine-et-Marne) & Bartonien & & & & (Eure) & \\
\hline A71417 & Faullummel & 1 & Dhuisy (Seine-et-Marne) & Bartonien & * & Six & 20 & Fontenay-en-Vexin & Lutétien \\
\hline $\begin{array}{l}\text { A57637 } \\
\text { B } 55188\end{array}$ & Faullummel & 5 & Dhuisy (Seine-et-Marne) & Bartonien & & & & (Eure) & \\
\hline B65188 & $1965-11$ & 1 & Vendrest (Seine-et- & Bartonien & & & & & \\
\hline
\end{tabular}


Annexe 1. - Suite

\begin{tabular}{|c|c|c|c|c|}
\hline No coll. & Coll. & $\begin{array}{l}\mathrm{Nb} \\
\text { spec. }\end{array}$ & Localités & Étages \\
\hline * & Boucher & 9 & $\begin{array}{l}\text { Fontenay-en-Vexin } \\
\text { (Eure) }\end{array}$ & Lutétien \\
\hline A57584 & MNHN & 1 & Vexin-sur-Epte (Eure) & Lutétien \\
\hline A57653 & Canevet & 1 & Requiécourt (Eure) & Lutétien \\
\hline A57593 & MNHN & 1 & Mont de Magny (Eure) & Lutétien \\
\hline B65169 & MNHN & 2 & Mont-de-Magny (Eure) & Lutétien \\
\hline A57627 & MNHN & 4 & Chaussy (Val d'Oise) & Lutétien \\
\hline * & Six & 3 & Chaussy (Val d'Oise) & Lutétien \\
\hline A57642 & Pacaud & 3 & Le Guépelle (Val d'Oise) & Bartonien \\
\hline A71468 & Pons & 1 & Le Guépelle (Val d'Oise) & Bartonien \\
\hline * & Boucher & 4 & Le Guépelle (Val d'Oise) & Bartonien \\
\hline * & Vyncke & 1 & Le Quoniam (Val d'Oise) & Bartonien \\
\hline * & Boucher & 1 & Le Quoniam (Val d'Oise) & Bartonien \\
\hline A57588 & Dollfus & 6 & $\begin{array}{l}\text { Thiverval-Grignon } \\
\text { (Yvelines) }\end{array}$ & Lutétien \\
\hline * & Sautereau & 8 & $\begin{array}{l}\text { Thiverval-Grignon } \\
\text { (Yvelines) }\end{array}$ & Lutétien \\
\hline A57651 & Péron & 16 & $\begin{array}{l}\text { Thiverval-Grignon } \\
\text { (Yvelines) }\end{array}$ & Lutétien \\
\hline B65170 & Morlet & 2 & $\begin{array}{c}\text { Thiverval-Grignon } \\
\text { (Yvelines) }\end{array}$ & Lutétien \\
\hline B65173 & MNHN & 21 & $\begin{array}{c}\text { Thiverval-Grignon } \\
\text { (Yvelines) }\end{array}$ & Lutétien \\
\hline B65174 & MNHN & 8 & $\begin{array}{l}\text { Thiverval-Grignon } \\
\text { (Yvelines) }\end{array}$ & Lutétien \\
\hline B69243 & MNHN & 1 & $\begin{array}{c}\text { Thiverval-Grignon } \\
\text { (Yvelines) }\end{array}$ & Lutétien \\
\hline A71410 & d'Orbigny & 1 & $\begin{array}{l}\text { Thiverval-Grignon } \\
\text { (Yvelines) }\end{array}$ & Lutétien \\
\hline A11519 & d'Orbigny & 5 & $\begin{array}{l}\text { Thiverval-Grignon } \\
\text { (Yvelines) }\end{array}$ & Lutétien \\
\hline A57590 & Brongniart & 7 & $\begin{array}{c}\text { Thiverval-Grignon } \\
\text { (Yvelines) }\end{array}$ & Lutétien \\
\hline A57591 & Brongniart & 1 & $\begin{array}{c}\text { Thiverval-Grignon } \\
\text { (Yvelines) }\end{array}$ & Lutétien \\
\hline A57592 & MNHN & 1 & $\begin{array}{l}\text { Thiverval-Grignon } \\
\text { (Yvelines) }\end{array}$ & Lutétien \\
\hline
\end{tabular}

\begin{tabular}{|c|c|c|c|c|}
\hline No coll. & Coll. & $\begin{array}{l}\mathrm{Nb} \\
\text { spec. }\end{array}$ & Localités & Étages \\
\hline A57625 & MNHN & 3 & $\begin{array}{c}\text { Thiverval-Grignon } \\
\text { (Yvelines) }\end{array}$ & Lutétien \\
\hline B65175 & Lhomme & 3 & $\begin{array}{c}\text { Thiverval-Grignon } \\
\text { (Yvelines) }\end{array}$ & Lutétien \\
\hline A70494 & Hoffstetter & 6 & $\begin{array}{c}\text { Thiverval-Grignon } \\
\text { (Yvelines) }\end{array}$ & Lutétien \\
\hline A70752 & de Muizon & 13 & $\begin{array}{l}\text { Thiverval-Grignon } \\
\text { (Yvelines) }\end{array}$ & Lutétien \\
\hline * & Vyncke & 25 & $\begin{array}{l}\text { Thiverval-Grignon } \\
\text { (Yvelines) }\end{array}$ & Lutétien \\
\hline A57635 & Pacaud & 1 & $\begin{array}{l}\text { Villiers-Saint-Frédéric } \\
\text { (Yvelines) }\end{array}$ & Lutétien \\
\hline A71470 & Pons & 2 & $\begin{array}{l}\text { Villiers-Saint-Frédéric } \\
\text { (Yvelines) }\end{array}$ & Lutétien \\
\hline A71657 & Faullummel & 1 & $\begin{array}{l}\text { Villiers-Saint-Frédéric } \\
\text { (Yvelines) }\end{array}$ & Lutétien \\
\hline * & Six & 40 & $\begin{array}{l}\text { Villiers-Saint.-Frédéric } \\
\text { (Yvelines) }\end{array}$ & Lutétien \\
\hline * & Boucher & 7 & $\begin{array}{l}\text { Villiers-Saint-Frédéric } \\
\text { (Yvelines) }\end{array}$ & Lutétien \\
\hline B65178 & $1965-11$ & 1 & $\begin{array}{l}\text { Saulx-Marchais } \\
\text { (Yvelines) }\end{array}$ & Lutétien \\
\hline * & Six & 32 & $\begin{array}{l}\text { Saulx-Marchais } \\
\text { (Yvelines) }\end{array}$ & Lutétien \\
\hline A71653 & Mouchart & 5 & $\begin{array}{l}\text { Beynes [La Remise à } \\
\text { Boucher] (Yvelines) } \\
\text { Lutétien }\end{array}$ & \\
\hline * & Boucher & 1 & $\begin{array}{l}\text { Beynes [La Remise à } \\
\text { Boucher] (Yvelines) } \\
\text { Lutétien }\end{array}$ & \\
\hline A57632 & Morlet & 3 & Montainville (Yvelines) & Lutétien \\
\hline & Sautereau & 2 & Maulette (Yvelines) & Lutétien \\
\hline A57655 & Pacaud & 2 & $\begin{array}{l}\text { Bois-Gouët (Loire- } \\
\text { Atlantique) }\end{array}$ & Bartonien \\
\hline A71244 & Braillon & 12 & Localité inconnue & Lutétien \\
\hline
\end{tabular}


ANNEXE 2. - Cryptochorda (s.str.) altavesna n. sp., liste du matériel étudié (437 exemplaires). Abréviations: $\mathbf{N}^{\circ}$, numéro du spécimen correspondant dans les collections de Paléontologie du Muséum (MNHN.F); Coll., nom de la collection; Nb spec., nombre de spécimens.

\begin{tabular}{|c|c|c|c|c|c|c|c|c|c|}
\hline No coll. & Coll. & $\begin{array}{l}\mathrm{Nb} \\
\text { spec. }\end{array}$ & Localités & Étages & No coll. & Coll. & $\begin{array}{l}\text { Nb } \\
\text { spec. }\end{array}$ & Localités & Étages \\
\hline $\begin{array}{l}\text { Holotype } \\
\text { A71419 }\end{array}$ & Sautereau & 1 & Authevernes (Eure) & Lutétien & $\begin{array}{l}\text { A57654 } \\
\text { A71472 }\end{array}$ & $\begin{array}{l}\text { Canevet } \\
\text { Sautereau }\end{array}$ & $\begin{array}{l}1 \\
4\end{array}$ & $\begin{array}{l}\text { Requiécourt (Eure) } \\
\text { Requiécourt (Eure) }\end{array}$ & $\begin{array}{l}\text { Lutétien } \\
\text { Lutétien }\end{array}$ \\
\hline $\begin{array}{l}\text { Paratype } \\
\text { A71420 }\end{array}$ & Sautereau & 1 & Authevernes (Eure) & Lutétien & * & $\begin{array}{l}\text { Sautereau } \\
\text { Sautereau }\end{array}$ & $\begin{array}{l}6 \\
1\end{array}$ & $\begin{array}{l}\text { Requiécourt (Eure) } \\
\text { Berchères/Vesgre }\end{array}$ & $\begin{array}{l}\text { Lutétien } \\
\text { Lutétien }\end{array}$ \\
\hline $\begin{array}{l}\text { Paratype } \\
\text { A71423 }\end{array}$ & Sautereau & 1 & Authevernes (Eure) & Lutétien & A57646 & de Roissy & 64 & $\begin{array}{l}\text { (Eure-et-Loir) } \\
\text { Parnes (Oise) }\end{array}$ & Lutétien \\
\hline $\begin{array}{l}\text { Paratype } \\
\text { A71424 }\end{array}$ & Sautereau & 1 & Authevernes (Eure) & Lutétien & $\begin{array}{l}\text { A71429 } \\
\text { A71683 }\end{array}$ & $\begin{array}{l}\text { de Roissy } \\
\text { de Roissy }\end{array}$ & $\begin{array}{r}1 \\
24\end{array}$ & $\begin{array}{l}\text { Parnes (Oise) } \\
\text { Parnes (Oise) }\end{array}$ & $\begin{array}{l}\text { Lutétien } \\
\text { Lutétien }\end{array}$ \\
\hline $\begin{array}{l}\text { Paratype } \\
\text { A71425 }\end{array}$ & Sautereau & 1 & Authevernes (Eure) & Lutétien & $\begin{array}{l}\text { A71684 } \\
\text { A71430 }\end{array}$ & $\begin{array}{l}\text { de Roissy } \\
\text { de Roissy }\end{array}$ & $\begin{array}{r}27 \\
1\end{array}$ & $\begin{array}{l}\text { Parnes (Oise) } \\
\text { Parnes (Oise) }\end{array}$ & $\begin{array}{l}\text { Lutétien } \\
\text { Lutétien }\end{array}$ \\
\hline $\begin{array}{l}\text { Paratype } \\
\text { A71426 }\end{array}$ & Sautereau & 1 & Authevernes (Eure) & Lutétien & $\begin{array}{l}\text { A71431 } \\
\text { A714421 }\end{array}$ & $\begin{array}{l}\text { de Roissy } \\
\text { de Roissy }\end{array}$ & $\begin{array}{l}1 \\
1\end{array}$ & $\begin{array}{l}\text { Parnes (Oise) } \\
\text { Parnes (Oise) }\end{array}$ & $\begin{array}{l}\text { Lutétien } \\
\text { Lutétien }\end{array}$ \\
\hline $\begin{array}{l}\text { Paratype } \\
\text { A71427 }\end{array}$ & Sautereau & 1 & Authevernes (Eure) & Lutétien & $\begin{array}{l}\text { A71422 } \\
\text { A57647 }\end{array}$ & $\begin{array}{l}\text { de Roissy } \\
\text { Brongniart }\end{array}$ & $\begin{array}{l}1 \\
1\end{array}$ & $\begin{array}{l}\text { Parnes (Oise) } \\
\text { Parnes (Oise) }\end{array}$ & $\begin{array}{l}\text { Lutétien } \\
\text { Lutétien }\end{array}$ \\
\hline Paratype & Sautereau & 1 & Authevernes (Eure) & Lutétien & $\begin{array}{l}\text { A57648 } \\
\text { B65182 }\end{array}$ & $\begin{array}{l}\text { d'Orbigny } \\
\text { Sarazin }\end{array}$ & $\begin{array}{l}1 \\
3\end{array}$ & $\begin{array}{l}\text { Parnes (Oise) } \\
\text { Parnes (Oise) }\end{array}$ & $\begin{array}{l}\text { Lutétien } \\
\text { Lutétien }\end{array}$ \\
\hline $\begin{array}{l}\text { A/1432 } \\
\text { Paratype } \\
\text { P71433 }\end{array}$ & Sautereau & 4 & Authevernes (Eure) & Lutétien & $\begin{array}{l}\text { A71659 } \\
\text { A80236 }\end{array}$ & $\begin{array}{l}\text { Lhomme } \\
\text { MNHN }\end{array}$ & $\begin{array}{l}1 \\
3\end{array}$ & $\begin{array}{l}\text { Parnes (Oise) } \\
\text { Parnes (Oise) }\end{array}$ & $\begin{array}{l}\text { Lutétien } \\
\text { Lutétien }\end{array}$ \\
\hline $\begin{array}{l}\text { A71433 } \\
\text { A57645 }\end{array}$ & Sautereau & 25 & Authevernes (Eure) & Lutétien & A71660 & Péron & 2 & Parnes (Oise) & Lutétien \\
\hline * & Sautereau & 169 & Authevernes (Eure) & Lutétien & A27248 & Hoffstetter & 3 & Parnes (Oise) & Lutétien \\
\hline * & Sautereau & 6 & Authevernes (Eure) & Lutétien & $\underset{*}{\text { A80235 }}$ & $\begin{array}{l}\text { MNHN } \\
\text { Boucher }\end{array}$ & $\begin{array}{l}3 \\
2\end{array}$ & $\begin{array}{l}\text { Parnes (Oise) } \\
\text { Parnes (Oise) }\end{array}$ & $\begin{array}{l}\text { Lutétien } \\
\text { Lutétien }\end{array}$ \\
\hline * & $\begin{array}{l}\text { Six } \\
\text { Boucher }\end{array}$ & $\begin{array}{l}10 \\
10\end{array}$ & $\begin{array}{l}\text { Authevernes (Eure) } \\
\text { Authevernes (Eure) }\end{array}$ & $\begin{array}{l}\text { Lutétien } \\
\text { Lutétien }\end{array}$ & A71676 & $\begin{array}{l}\text { Boucher } \\
\text { MNHN }\end{array}$ & $\begin{array}{l}2 \\
2\end{array}$ & Chaumont-en-Vexin & $\begin{array}{l}\text { Lutetien } \\
\text { Lutétien }\end{array}$ \\
\hline A71434 & Pacaud & 1 & $\begin{array}{l}\text { Fontenay-en-Vexin } \\
\text { (Eure) }\end{array}$ & Lutétien & B65171 & Morlet & 1 & Thiverval-Grignon & Lutétien \\
\hline A71435 & Pacaud & 1 & $\begin{array}{l}\text { Fontenay-en-Vexin } \\
\text { (Eure) }\end{array}$ & Lutétien & B70690 & d'Orbigny & 2 & $\begin{array}{l}\text { (Yvelines) } \\
\text { Thiverval-Grignon }\end{array}$ & Lutétien \\
\hline A71436 & Pacaud & 1 & $\begin{array}{l}\text { Fontenay-en-Vexin } \\
\text { (Eure) }\end{array}$ & Lutétien & A71673 & MNHN & 1 & $\begin{array}{l}\text { (Yvelines) } \\
\text { Thiverval-Grignon }\end{array}$ & Lutétien \\
\hline A71437 & Pacaud & 1 & $\begin{array}{l}\text { Fontenay-en-Vexin } \\
\text { (Eure) }\end{array}$ & Lutétien & A71677 & MNHN & 2 & $\begin{array}{l}\text { (Yvelines) } \\
\text { Thiverval-Grignon }\end{array}$ & Lutétien \\
\hline A71438 & Pacaud & 1 & $\begin{array}{l}\text { Fontenay-en-Vexin } \\
\text { (Eure) }\end{array}$ & Lutétien & * & Sautereau & 4 & Thiverval-Grignon & Lutétien \\
\hline A71439 & Pacaud & 1 & $\begin{array}{l}\text { Fontenay-en-Vexin } \\
\text { (Eure) }\end{array}$ & Lutétien & * & Vyncke & 2 & $\begin{array}{l}\text { (Yvelines) } \\
\text { Thiverval-Grignon }\end{array}$ & Lutétien \\
\hline A71440 & Pacaud & 1 & $\begin{array}{l}\text { Fontenay-en-Vexin } \\
\text { (Eure) }\end{array}$ & Lutétien & A71661 & Sautereau & 1 & $\begin{array}{l}\text { (Yvelines) } \\
\text { Beynes [La Remise à }\end{array}$ & \\
\hline A71441 & Pacaud & 1 & $\begin{array}{l}\text { Fontenay-en-Vexin } \\
\text { (Eure) }\end{array}$ & Lutétien & & & & $\begin{array}{l}\text { Boucher] (Yvelines) } \\
\text { Lutétien }\end{array}$ & \\
\hline A71674 & Pacaud & 7 & $\begin{array}{l}\text { Fontenay-en-Vexin } \\
\text { (Eure) }\end{array}$ & Lutétien & $\begin{array}{l}\text { A57649 } \\
\text { A71428 }\end{array}$ & $\begin{array}{l}\text { Morlet } \\
\text { Sautereau }\end{array}$ & $\begin{array}{l}1 \\
1\end{array}$ & $\begin{array}{l}\text { Montainville (Yvelines) } \\
\text { Maulette (Yvelines) }\end{array}$ & $\begin{array}{l}\text { Lutétien } \\
\text { Lutétien }\end{array}$ \\
\hline$\underset{*}{\text { A71678 }}$ & $\begin{array}{l}\text { Pacaud } \\
\text { Pacaud }\end{array}$ & $\begin{array}{l}7 \\
1\end{array}$ & $\begin{array}{l}\text { Fontenay-en-Vexin (Eure) } \\
\text { Fontenay-en-Vexin (Eure) }\end{array}$ & $\begin{array}{l}\text { Lutétien } \\
\text { Lutétien }\end{array}$ & $\begin{array}{l}\text { A71471 } \\
\star\end{array}$ & $\begin{array}{l}\text { Sautereau } \\
\text { Sautereau }\end{array}$ & $\begin{array}{r}4 \\
10 \\
\end{array}$ & $\begin{array}{l}\text { Maulette (Yvelines) } \\
\text { Maulette (Yvelines) }\end{array}$ & $\begin{array}{l}\text { Lutétien } \\
\text { Lutétien }\end{array}$ \\
\hline
\end{tabular}

ANNEXE 3. - Cryptochorda (s.str.) pyrenaicensis Cossmann, 1923, liste du matériel étudié (7 exemplaires). Abréviations: $\mathbf{N}^{\circ}$, numéro du spécimen correspondant dans les collections de Paléontologie du Muséum (MNHN.F); Coll., nom de la collection; Nb spec., nombre de spécimens.

\begin{tabular}{|c|c|c|c|c|}
\hline No coll. & Coll. & $\begin{array}{l}\text { Nb } \\
\text { spec. }\end{array}$ & Localités & Étages \\
\hline J14638 & Cossmann & & $\begin{array}{l}\text { Gan (Pyrénées- } \\
\text { Atlantiques) } \\
\text { Gan (Pyrénées- }\end{array}$ & Cuisien \\
\hline J10882 & O'Gorman & & $\begin{array}{l}\text { Atlantiques) } \\
\text { Gan (Pyrénées- }\end{array}$ & Cuisien \\
\hline A71481 & O'Gorman & & $\begin{array}{l}\text { Atlantiques) } \\
\text { Gan (Pyrénées- }\end{array}$ & Cuisien \\
\hline A57359 & Pacaud & 1 & $\begin{array}{l}\text { Atlantiques) } \\
\text { Gan (Pyrénées- }\end{array}$ & Cuisien \\
\hline $\begin{array}{l}\text { A71775 } \\
\text { UBT 29-2- } \\
125\end{array}$ & $\begin{array}{l}\text { Varone } \\
\text { O'Gorman }\end{array}$ & $\begin{array}{l}1 \\
2\end{array}$ & $\begin{array}{l}\text { Atlantiques) } \\
\text { Gan (Pyrénées- } \\
\text { Atlantiques) }\end{array}$ & $\begin{array}{l}\text { Cuisien } \\
\text { Cuisien }\end{array}$ \\
\hline
\end{tabular}


ANNEXE 4. - Cryptochorda (s.str.) neptis n. sp., liste du matériel étudié [214 exemplaires]. Abréviations: $\mathbf{N}^{\circ}$, numéro du spécimen correspondant dans les collections de Paléontologie du Muséum (MNHN.F); Coll., nom de la collection; Nb spec., nombre de spécimens.

\begin{tabular}{|c|c|c|c|c|}
\hline No coll. & Coll. & $\begin{array}{l}\mathrm{Nb} \\
\text { spec. }\end{array}$ & Localités & Étages \\
\hline $\begin{array}{l}\text { Holotype } \\
\text { A71442 }\end{array}$ & Pacaud & 1 & Saint-Gobain (Aisne) & Cuisien \\
\hline $\begin{array}{l}\text { Paratype } \\
\text { A71443 }\end{array}$ & Pacaud & 1 & Saint-Gobain (Aisne) & Cuisien \\
\hline $\begin{array}{l}\text { Paratype } \\
\text { A71444 }\end{array}$ & Pacaud & 1 & Saint-Gobain (Aisne) & Cuisien \\
\hline $\begin{array}{l}\text { Paratype } \\
\text { A71446 }\end{array}$ & Pacaud & 1 & Saint-Gobain (Aisne) & Cuisien \\
\hline $\begin{array}{l}\text { Paratype } \\
\text { A71449 }\end{array}$ & Pacaud & 1 & Saint-Gobain (Aisne) & Cuisien \\
\hline $\begin{array}{l}\text { Paratype } \\
\text { A71465 }\end{array}$ & Pons & 1 & Saint-Gobain (Aisne) & Cuisien \\
\hline $\begin{array}{l}\text { Paratype } \\
\text { A71445 }\end{array}$ & Romanek & 1 & Saint-Gobain (Aisne) & Cuisien \\
\hline $\begin{array}{l}\text { Paratype } \\
\text { A71447 }\end{array}$ & Romanek & 1 & Saint-Gobain (Aisne) & Cuisien \\
\hline $\begin{array}{l}\text { Paratype } \\
\text { A71448 }\end{array}$ & Romanek & 1 & Saint-Gobain (Aisne) & Cuisien \\
\hline $\begin{array}{l}\text { Paratype } \\
\text { A71450 }\end{array}$ & Romanek & 1 & Saint-Gobain (Aisne) & Cuisien \\
\hline $\begin{array}{l}\text { Paratype } \\
\text { A71451 }\end{array}$ & Romanek & 1 & Saint-Gobain (Aisne) & Cuisien \\
\hline A71455 & Pacaud & 1 & Saint-Gobain (Aisne) & Cuisien \\
\hline J14640 & Cossmann & 3 & Saint-Gobain (Aisne) & Cuisien \\
\hline A71479 & $\begin{array}{l}\text { ex-Galerie } \\
\text { de } \\
\text { Zoologie }\end{array}$ & 2 & Saint-Gobain (Aisne) & Cuisien \\
\hline B65161 & Lhomme & 1 & Saint-Gobain (Aisne) & Cuisien \\
\hline A71477 & Faullummel & 115 & Saint-Gobain (Aisne) & Cuisien \\
\hline A71482 & Romanek & 14 & Saint-Gobain (Aisne) & Cuisien \\
\hline * & Romanek & 20 & Saint-Gobain (Aisne) & Cuisien \\
\hline * & Vyncke & 12 & Saint-Gobain (Aisne) & Cuisien \\
\hline * & Boucher & 25 & Saint-Gobain (Aisne) & Cuisien \\
\hline * & Six & 29 & Saint-Gobain (Aisne) & Cuisien \\
\hline * & Vyncke & 12 & Saint-Gobain (Aisne) & Cuisien \\
\hline * & Boucher & 21 & Saint-Gobain (Aisne) & Cuisien \\
\hline * & Vyncke & 1 & Laon (Aisne) & Cuisien \\
\hline A71473 & Faullummel & & Laversines (Aisne) & Cuisien \\
\hline A71480 & Faullummel & 1 & Monampteuil (Aisne) & Cuisien \\
\hline B65162 & Lhomme & 3 & $\begin{array}{l}\text { Mons-en-Laonnois } \\
\text { (Aisne) }\end{array}$ & Cuisien \\
\hline A71452 & Lhomme & 1 & $\begin{array}{l}\text { Mons-en-Laonnois } \\
\text { (Aisne) }\end{array}$ & Cuisien \\
\hline A71453 & Lhomme & 1 & $\begin{array}{l}\text { Mons-en-Laonnois } \\
\text { (Aisne) }\end{array}$ & Cuisien \\
\hline A71454 & Lhomme & 1 & $\begin{array}{l}\text { Mons-en-Laonnois } \\
\text { (Aisne) }\end{array}$ & Cuisien \\
\hline A71474 & de Morgan & 3 & $\begin{array}{l}\text { Mons-en-Laonnois } \\
\text { (Aisne) }\end{array}$ & Cuisien \\
\hline A71466 & Pons & 7 & Mercin-et-Vaux (Aisne) & Cuisien \\
\hline A71478 & Faullummel & 11 & Mercin-et-Vaux (Aisne) & Cuisien \\
\hline * & Boucher & 4 & Mercin-et-Vaux (Aisne) & Cuisien \\
\hline * & Six & 7 & Lizy (Aisne) & Cuisien \\
\hline * & Boucher & 3 & $\begin{array}{l}\text { Bruyères-et- } \\
\text { Montbérault (Aisne) }\end{array}$ & Cuisien \\
\hline B65163 & Lhomme & 1 & Cuise-la-Motte (Oise) & Cuisien \\
\hline A71476 & $\begin{array}{l}\text { ex-Galerie } \\
\text { de } \\
\text { Zoologie }\end{array}$ & 4 & Cuise-la-Motte (Oise) & Cuisien \\
\hline A10519 & d'Orbigny & 2 & Cuise-la-Motte (Oise) & Cuisien \\
\hline A71475 & Faullummel & 4 & Cinqueux (Oise) & Cuisien \\
\hline A71462 & Merle & 1 & Egem (Belgique) & Cuisien \\
\hline A71463 & Merle & 1 & Egem (Belgique) & Cuisien \\
\hline A71464 & Merle & 3 & Egem (Belgique) & Cuisien \\
\hline
\end{tabular}

ANNEXE 5. - Cryptochorda (s.str.) cosediensis n. sp., liste du matériel étudié (29 exemplaires). Abréviations: $\mathbf{N}^{\circ}$, numéro du spécimen correspondant dans les collections de Paléontologie du Muséum (MNHN.F); Coll., nom de la collection; Nb spec., nombre de spécimens.

\begin{tabular}{|c|c|c|c|c|}
\hline No coll. & Coll. & $\begin{array}{l}\mathrm{Nb} \\
\text { spec. }\end{array}$ & Localités & Étages \\
\hline $\begin{array}{l}\text { Holotype } \\
\text { B65189 }\end{array}$ & de Morgan & 1 & Fresville (Manche) & Lutétien \\
\hline $\begin{array}{l}\text { Paratype } \\
\text { A05108 }\end{array}$ & Le Marchand & & Fresville (Manche) & Lutétien \\
\hline $\begin{array}{l}\text { Paratype } \\
\text { A71456 }\end{array}$ & de Morgan & 1 & Fresville (Manche) & Lutétien \\
\hline $\begin{array}{l}\text { Paratype } \\
\text { A71457 }\end{array}$ & de Morgan & 1 & Fresville (Manche) & Lutétien \\
\hline $\begin{array}{l}\text { Paratype } \\
\text { A71461 }\end{array}$ & de Morgan & 1 & Fresville (Manche) & Lutétien \\
\hline $\begin{array}{l}\text { Paratype } \\
\text { A71483 }\end{array}$ & de Morgan & 1 & Fresville (Manche) & Lutétien \\
\hline $\begin{array}{l}\text { Paratype } \\
\mathrm{J} 14642\end{array}$ & Cossmann & 1 & Fresville (Manche) & Lutétien \\
\hline A71458 & MNHN & 1 & Fresville (Manche) & Lutétien \\
\hline * & Ledon & 2 & Fresville (Manche) & Lutétien \\
\hline * & Tadeusz & 1 & Fresville (Manche) & Lutétien \\
\hline * & Sautereau & 9 & Fresville (Manche) & Lutétien \\
\hline A71459 & MNHN & 1 & $\begin{array}{l}\text { Hautteville-Bocage } \\
\text { (Manche) }\end{array}$ & Lutétien \\
\hline A71460 & MNHN & 1 & $\begin{array}{l}\text { Hautteville-Bocage } \\
\text { (Manche) }\end{array}$ & Lutétien \\
\hline B65190 & MNHN & 7 & $\begin{array}{l}\text { Hautteville-Bocage } \\
\text { (Manche) }\end{array}$ & Lutétien \\
\hline
\end{tabular}

